Supporting Information for...

\title{
C-N Coupling via Antiaromatic Endocyclic Nitrenium Ions
}

\author{
Shyamal Kanti Bera ${ }^{\mathrm{a}}$, Md Toufique Alam ${ }^{\mathrm{a}}$ and Prasenjit Mal ${ }^{\mathrm{a} *}$
}

${ }^{a}$ School of Chemical Sciences, National Institute of Science Education and Research (NISER), HBNI, Bhubaneswar, PO Bhimpur-Padanpur, Via Jatni, District Khurda, Odisha 752050, India; Tel: +919439613856; E-mail: pmal@niser.ac.in

\section{CONTENTS}

Crystallographic data S2-S3

References

NMR Spectra S5-S55

Mass Spectra S56-S57 


\section{X-ray crystallography analysis}

Procedure for preparing the crystal of 2-Fluoro-11,12-dimethylbenzo[4,5]imidazo[1,2f]phenanthridine (2o). In a $10 \mathrm{~mL}$ round bottom flask $20 \mathrm{mg}$ of 2-fluoro-11,12dimethylbenzo[4,5]imidazo[1,2-f]phenanthridine (2o) was dissolved by using ethyl acetate and hexane mixture $=1: 1(6 \mathrm{~mL})$. After that the solution was allowed for slow evaporation to obtain good quality of crystal of compound $2 \mathbf{0 .}$

\section{Crystal measurement:}

The crystals data were collected with Bruker SMART D8 goniometer equipped with an APEX CCD detector and with an INCOATEC micro source (Cu-Ka radiation, $\lambda=0.71073 \AA$ ). SAINT $+{ }^{1}$ and SADABS ${ }^{2}$ were used to integrate the intensities and to correct the absorption respectively The structure was resolved by direct methods and refined on $\mathrm{F}^{2}$ with SHELXL97. ${ }^{3}$ ORTEP Drawing of the compound $2 \mathbf{o}$ shows ellipsoid contour at the $50 \%$ probability level.

\section{Crystallographic data}
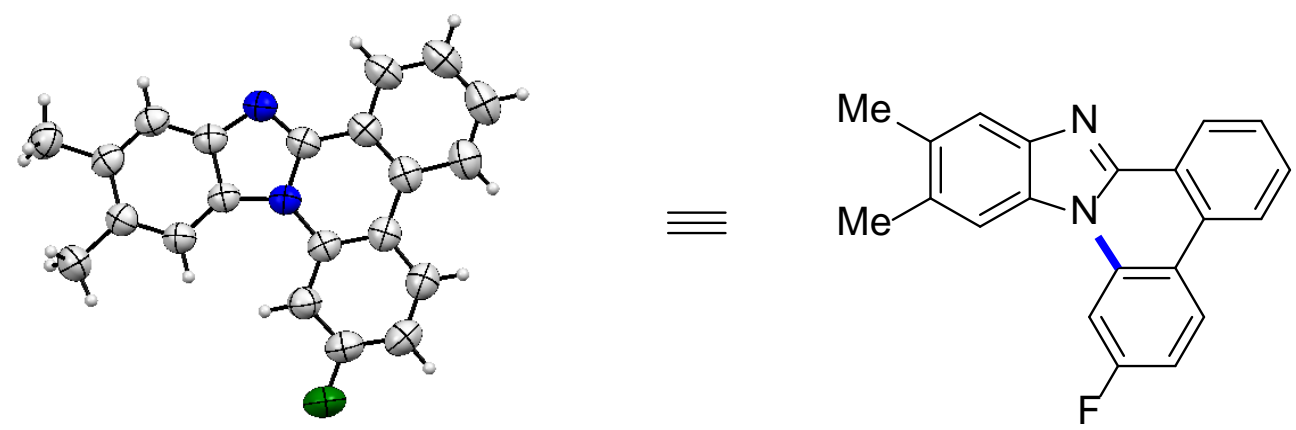

Figure S1. Crystal structure of $\mathbf{2 0}$ (50\% ellipsoid probability). 
Table S1. Crystal data and structure refinement for $2 \mathbf{0 .}$

CCDC No.

Chemical Formula

Formula Weight

Crystal system

Space group

Unit cell dimensions

Volume

Z

Density (calculated)

Crystal size

Final R indice [I>2sigma(I)]

$\mathrm{R}$ indices (all data)
1910734

$\mathrm{C}_{21} \mathrm{H}_{15} \mathrm{FN}_{2}$

314.35

triclinic

$\mathrm{P} 2{ }_{1} / \mathrm{C}$

$$
\begin{array}{ll}
\mathrm{a}=8.5984(3) \AA & \alpha=90^{\circ} . \\
\mathrm{b}=17.4730(5) \AA & \beta=110^{\circ} . \\
\mathrm{c}=10.8764(4) \AA & \gamma=90^{\circ} .
\end{array}
$$

1530.72(10) A3

4

$1.364 \mathrm{~g} / \mathrm{cm} 3$

$0.2,0.18,0.18$

$\mathrm{R}_{1}=0.0638, \mathrm{wR}_{2}=0.1703$

$\mathrm{R}_{1}=0.0666, \mathrm{wR}_{2}=0.1738$

\section{References}

(1) SAINT+, Bruker AXS Inc., Madison, Wisconsin, USA, 1999 (Program for Reduction of Data collected on Bruker CCD Area Detector Diffractometer V. 6.02.)

(2) SADABS, Bruker AXS, Madison, Wisconsin, USA, 2004

(3) Sheldrick, G., A short history of SHELX. Acta Crystallogr. Sect. A 2008, 64, 112-122. 
${ }^{1}$ H NMR (700 MHz, DMSO-d ${ }_{6}$ )

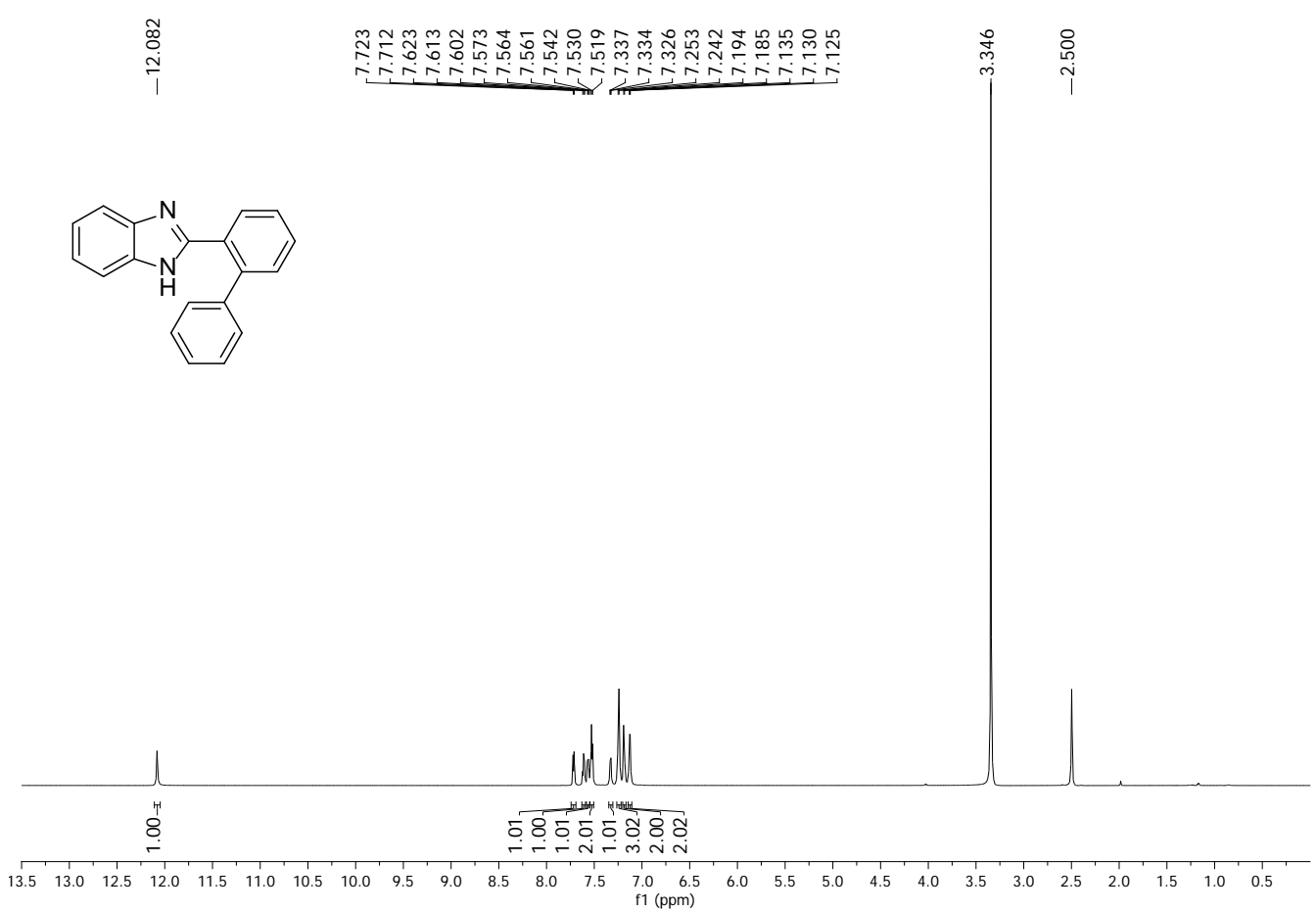

Figure S2. ${ }^{1} \mathrm{H}$ NMR spectrum of 2-([1,1'-Biphenyl]-2-yl)-1H-benzo[d]imidazole (1a).

${ }^{13}$ C NMR (175 MHz, DMSO-d 6 )

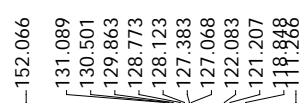

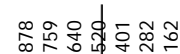
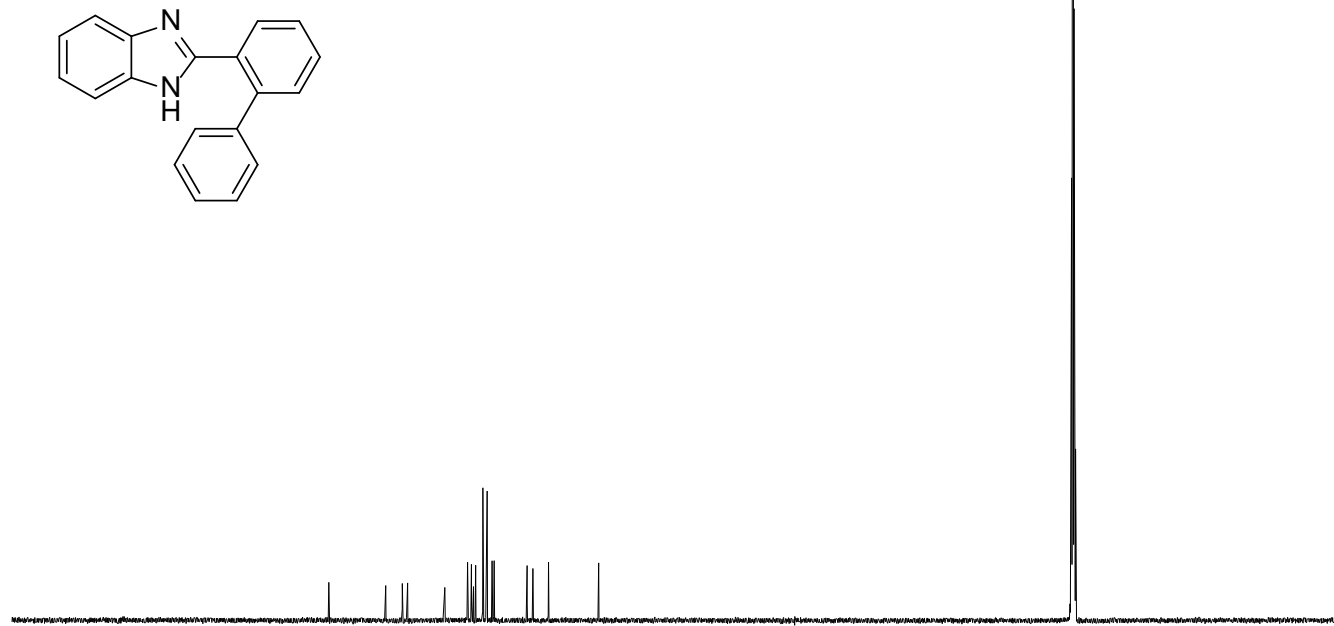

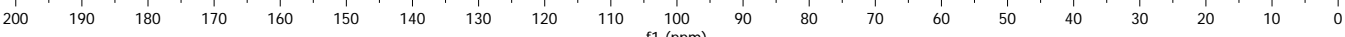

Figure S3. ${ }^{13} \mathrm{C}$ NMR spectrum of 2-([1,1'-Biphenyl]-2-yl)-1H-benzo[d]imidazole (1a). 
${ }^{1}$ H NMR (700 MHz, DMSO-d ${ }_{6}$ )

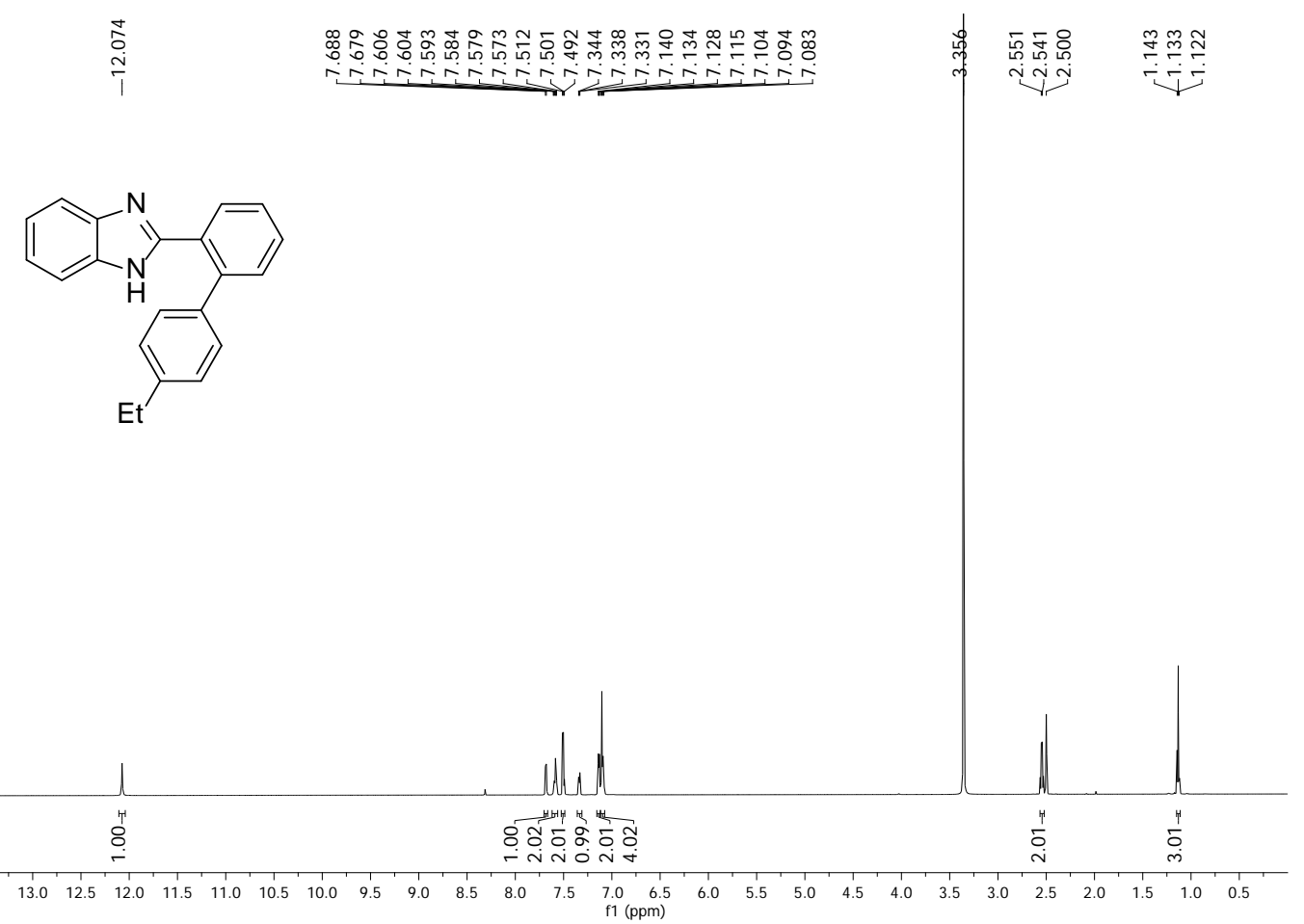

Figure S4. ${ }^{1} \mathrm{H}$ NMR spectrum of 2-(4'-Ethyl-[1,1'-biphenyl]-2-yl)-1H-benzo[d]imidazole (1b).

${ }^{13}$ C NMR (175 MHz, DMSO-d $\left.{ }_{6}\right)$
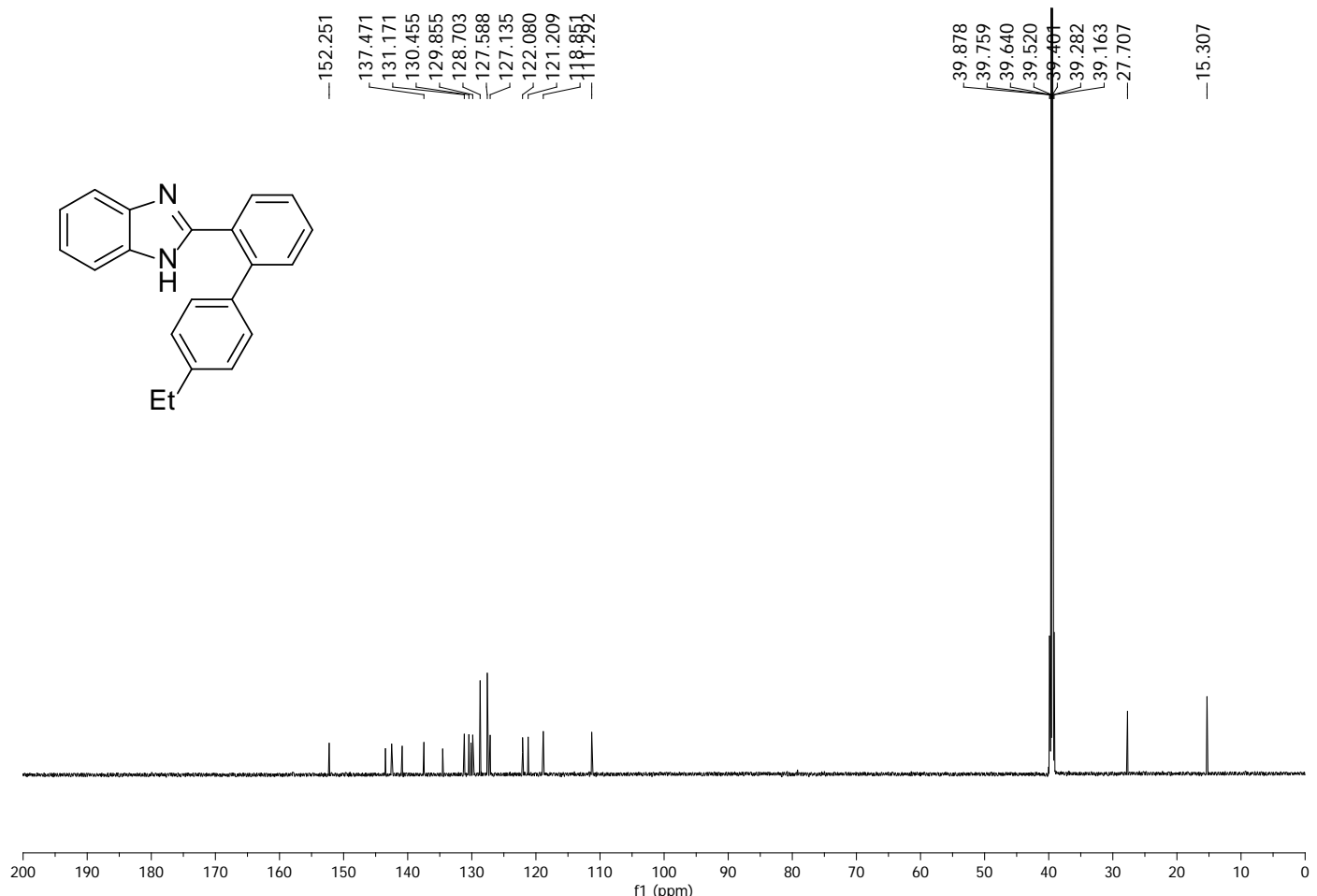

Figure S5. ${ }^{13} \mathrm{C}$ NMR spectrum of 2-(4'-Ethyl-[1,1'-biphenyl]-2-yl)-1H-benzo[d]imidazole (1b). 
${ }^{1}$ H NMR (700 MHz, DMSO-d $\left.{ }_{6}\right)$

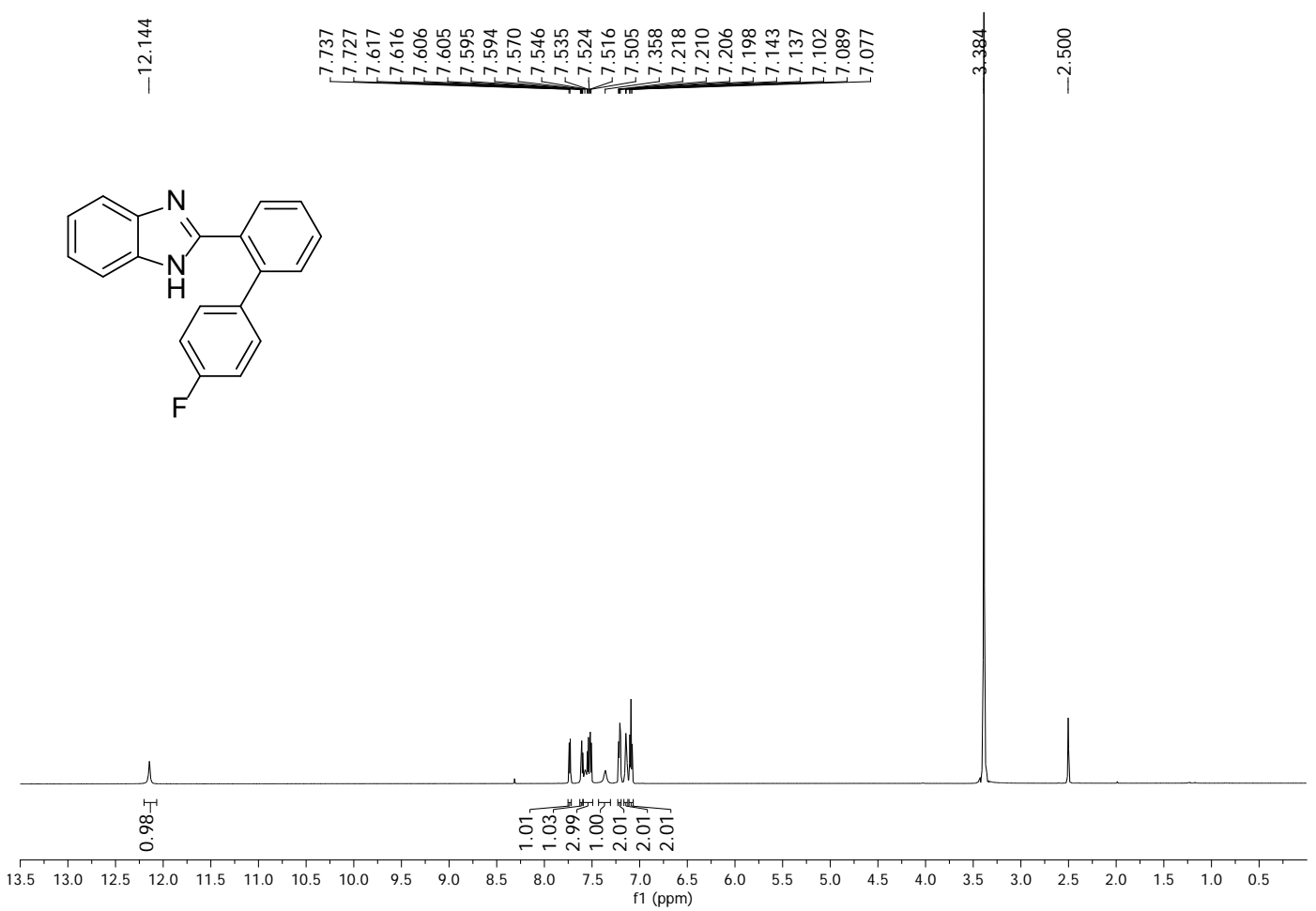

Figure S6. ${ }^{1} \mathrm{H}$ NMR spectrum of 2-(4'-Fluoro-[1,1'-biphenyl]-2-yl)-1H-benzo[d]imidazole (1c).

${ }^{13}$ C NMR (175 MHz, DMSO-d $)$
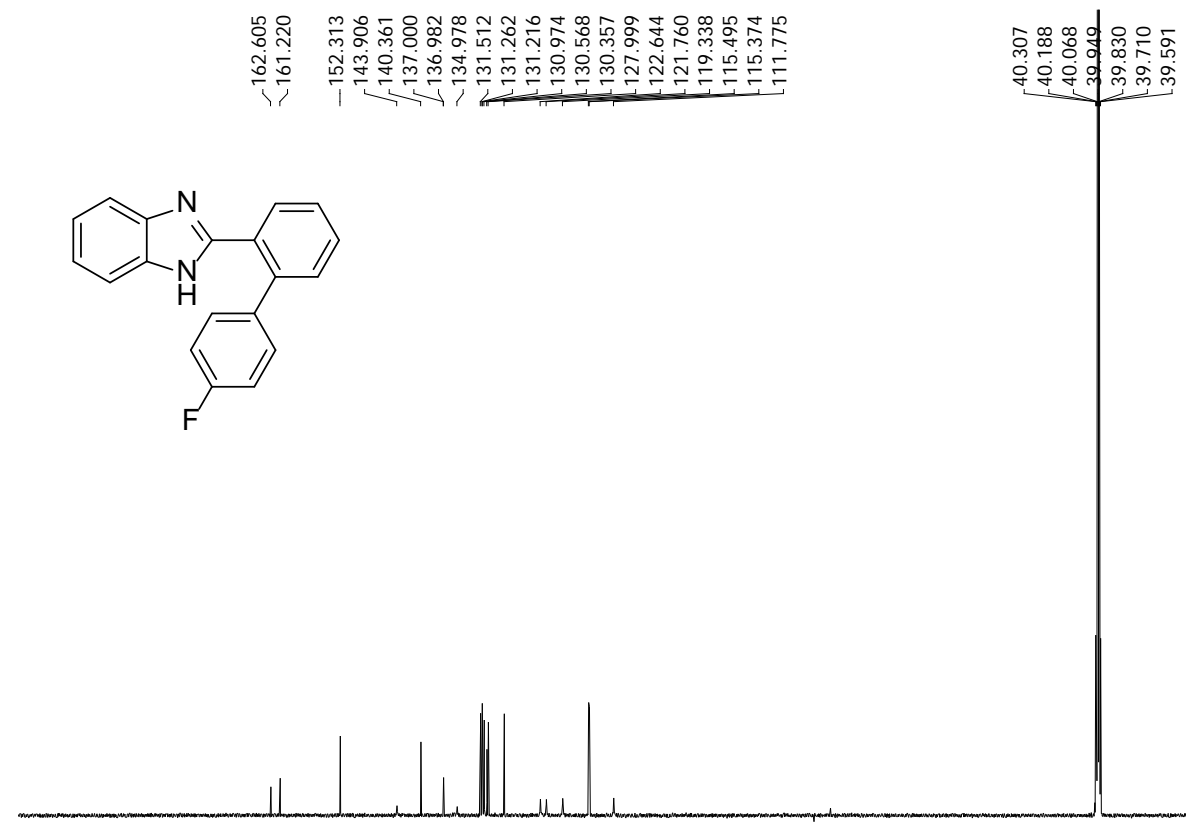

$\begin{array}{lllllllllllllllllllll}200 & 190 & 180 & 170 & 160 & 150 & 140 & 130 & 120 & 110 & 100 & 90 & 80 & 70 & 60 & 50 & 40 & 30 & 20 & 10 & 0\end{array}$

Figure S7. ${ }^{13} \mathrm{C}$ NMR spectrum of 2-(4'-Fluoro-[1,1'-biphenyl]-2-yl)-1H-benzo[d]imidazole (1c). 
${ }^{1}$ H NMR (700 MHz, DMSO-d $)$

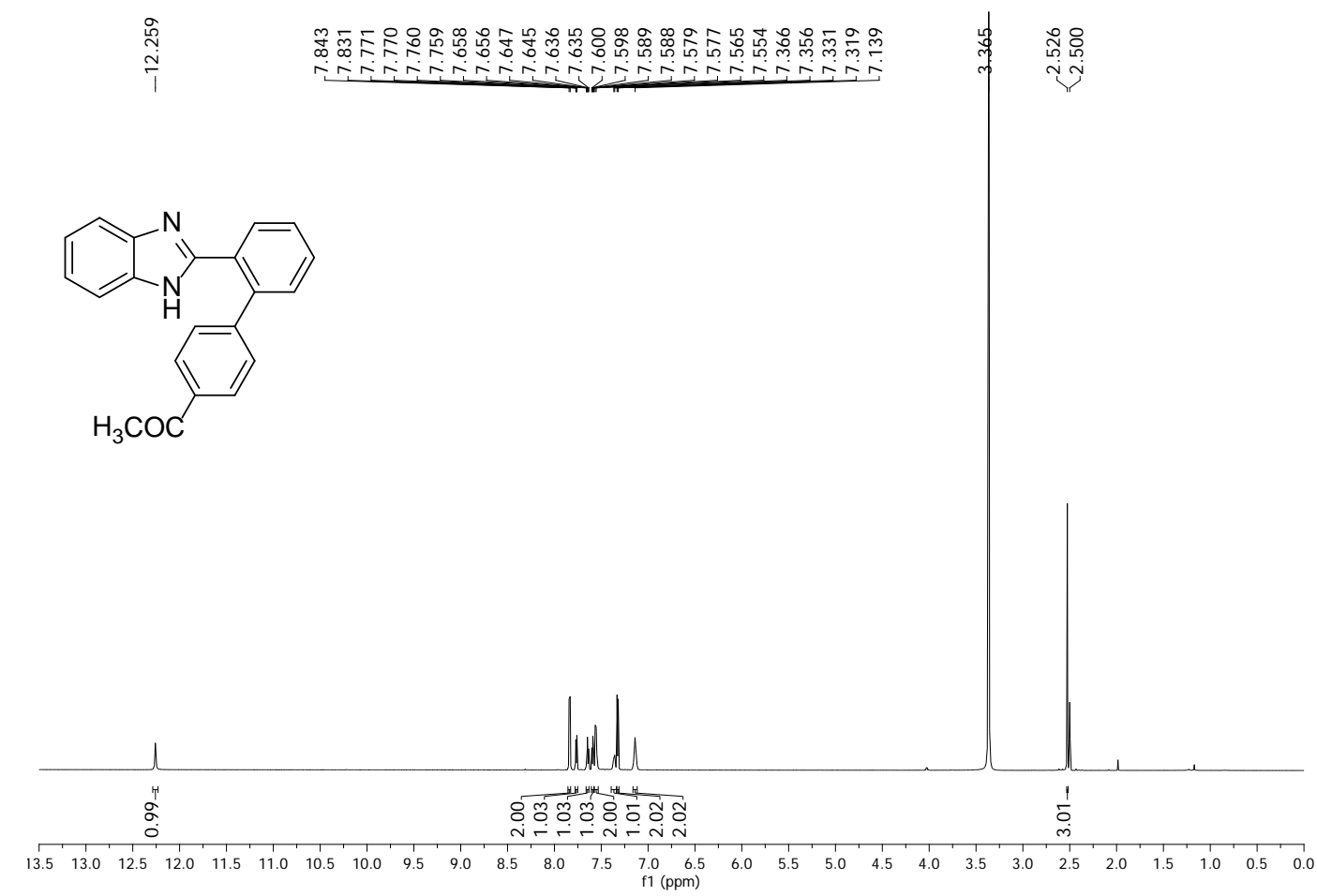

Figure S8. ${ }^{1} \mathrm{H}$ NMR spectrum of 1-(2'-(1H-Benzo[d]imidazol-2-yl)-[1,1'-biphenyl]-4yl)ethan-1-one (1d).

${ }^{13}$ C NMR (175 MHz, DMSO-d $)$

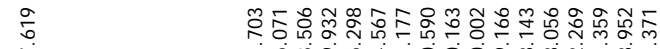

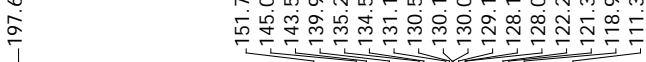

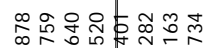

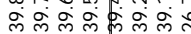<smiles>CC(=O)c1ccc(-c2ccccc2-c2nc3ccccc3[nH]2)cc1</smiles>

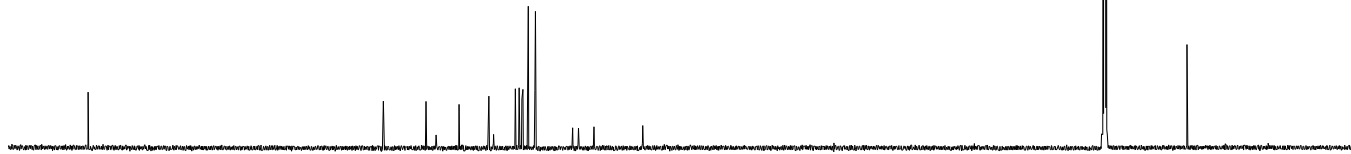

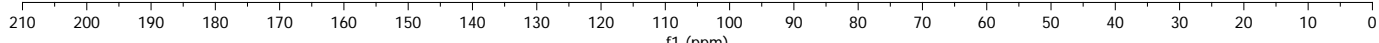

Figure S9. ${ }^{13} \mathrm{C}$ NMR spectrum of 1-(2'-(1H-Benzo[d]imidazol-2-yl)-[1,1'-biphenyl]-4yl)ethan-1-one (1d). 
${ }^{1}$ H NMR (700 MHz, DMSO-d $)_{6}$

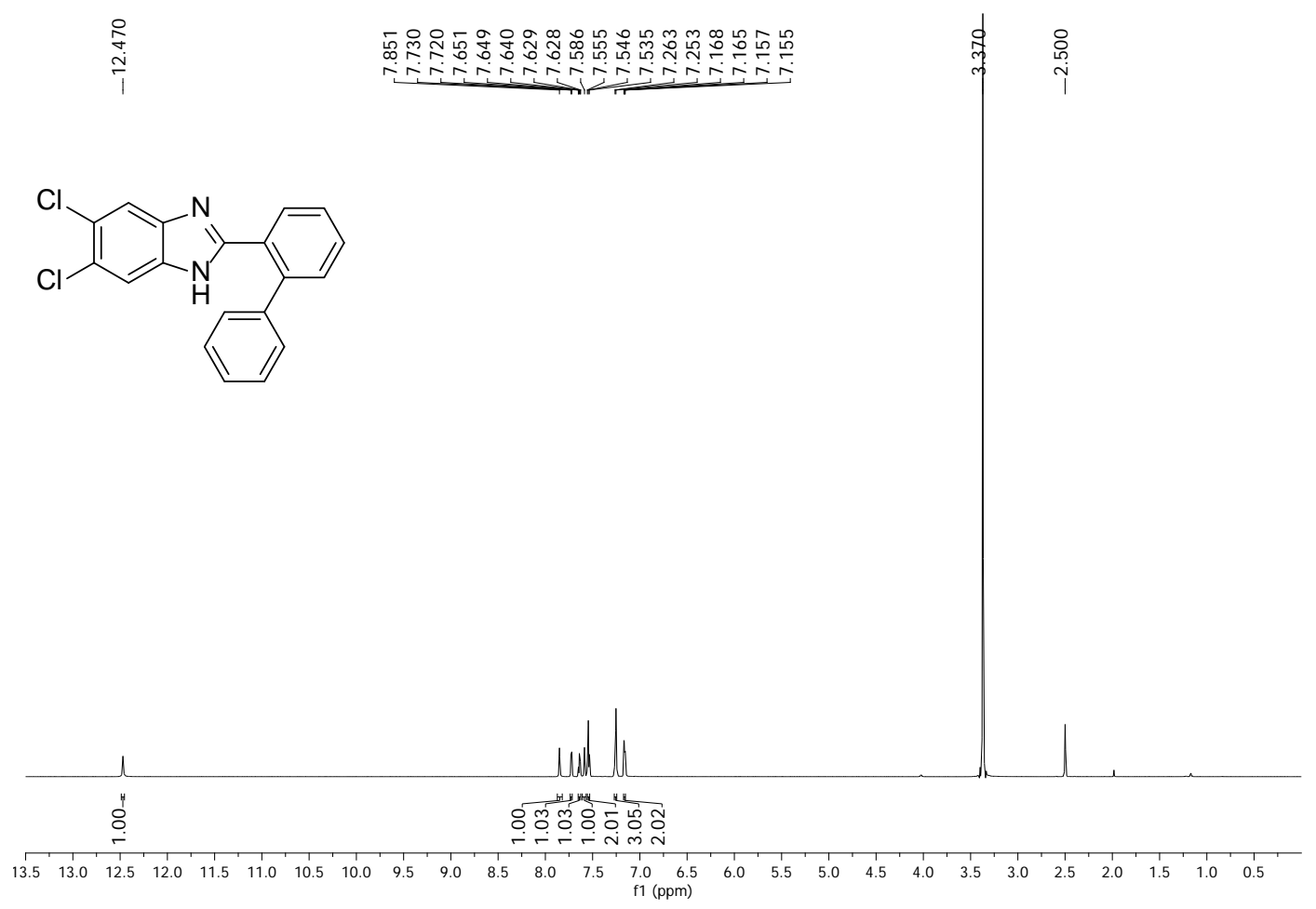

Figure S10. ${ }^{1} \mathrm{H}$ NMR spectrum of 2-([1,1'-Biphenyl]-2-yl)-5,6-dichloro-1Hbenzo[d]imidazole (1e)

${ }^{13}$ C NMR (175 MHz, DMSO-d $)$

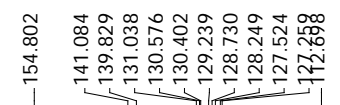

(l)

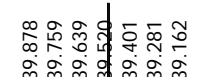

ㄱㄱㄱㄱㄱㄱㄱㄱㄱ
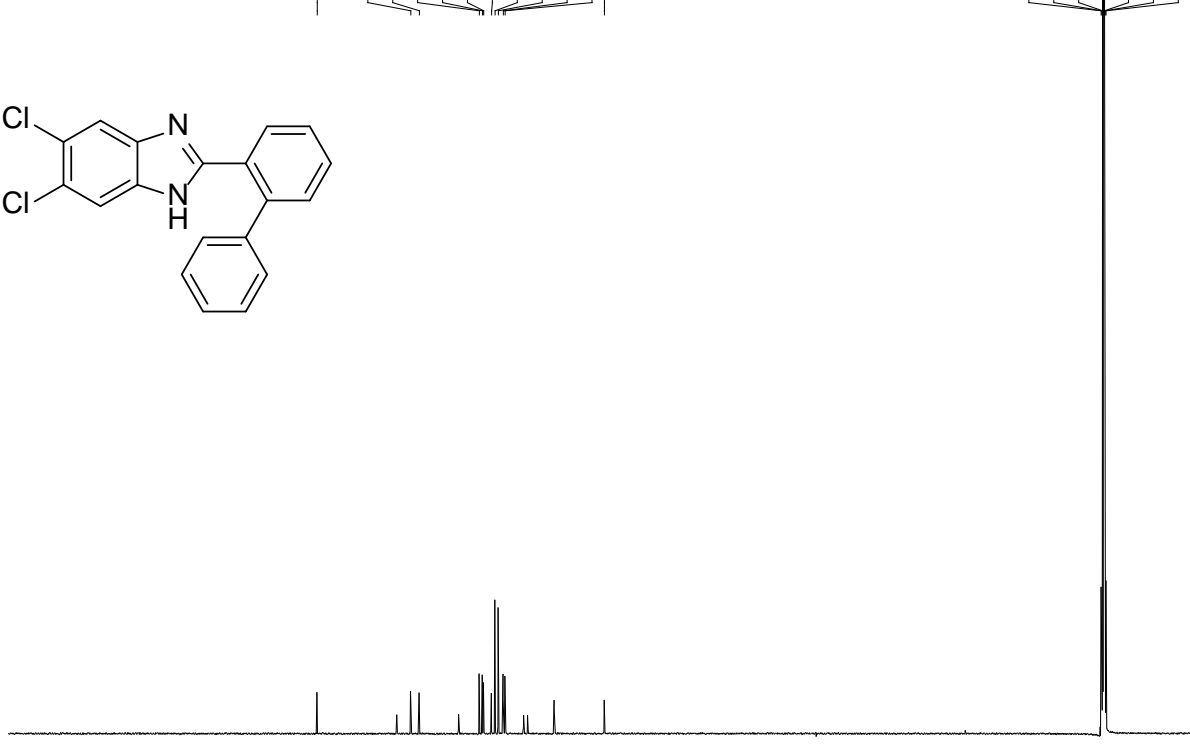

$\begin{array}{lllllllllllllllllllll}1 & 190 & 190 & 170 & 160 & 150 & 140 & 130 & 120 & 110 & \begin{array}{l}100 \\ \mathrm{f} 1(\mathrm{pm})\end{array} & 90 & 80 & 70 & 60 & 50 & 40 & 30 & 20 & 10\end{array}$

Figure S11. ${ }^{13} \mathrm{C}$ NMR spectrum of 2-([1,1'-Biphenyl]-2-yl)-5,6-dichloro-1Hbenzo[d]imidazole (1e). 
${ }^{1}$ H NMR (700 MHz, DMSO-d 6 )

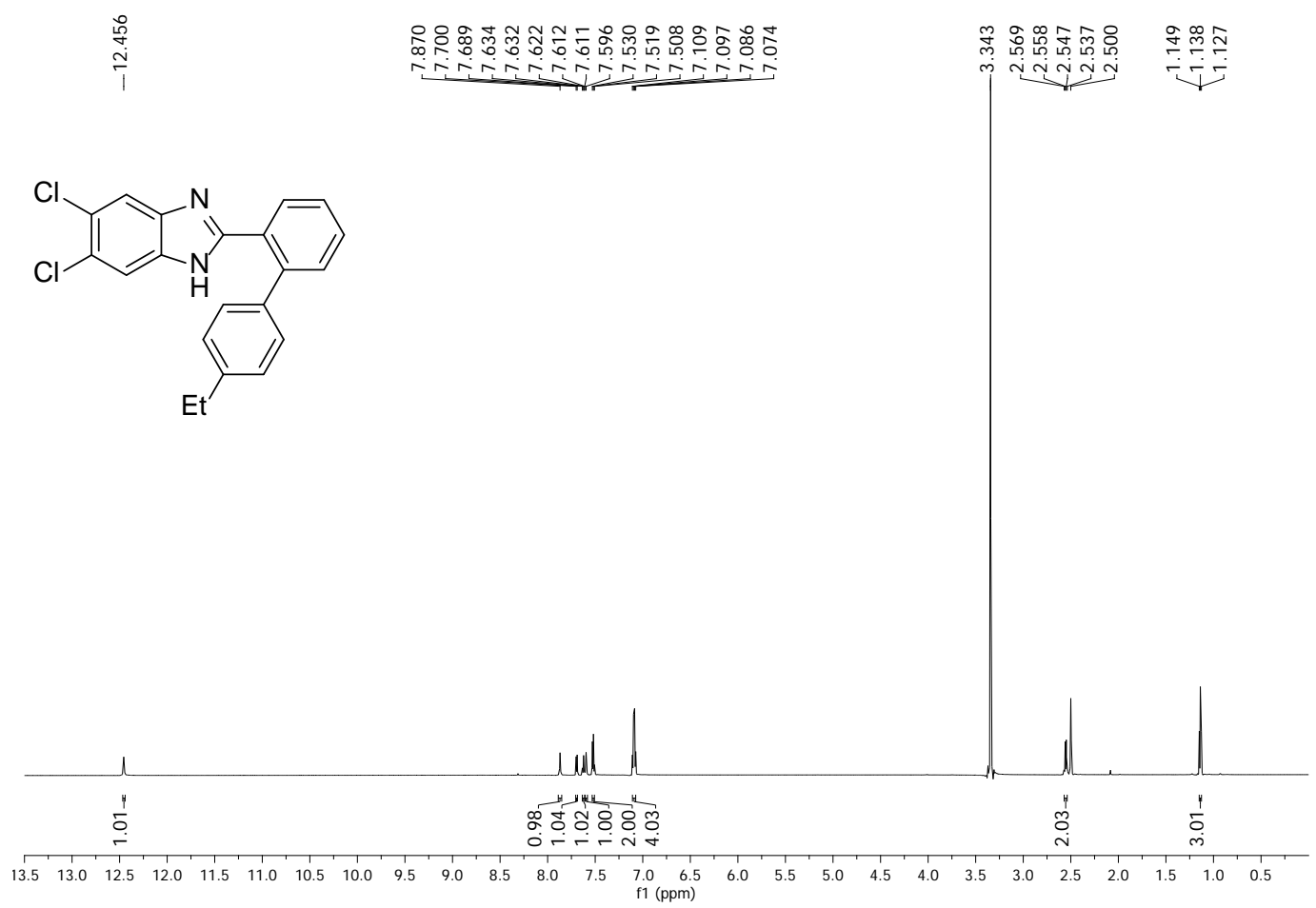

Figure S12. ${ }^{1} \mathrm{H}$ NMR spectrum of 5,6-dichloro-2-(4'-ethyl-[1,1'-biphenyl]-2-yl)-1Hbenzo[d]imidazole (1f).

${ }^{13}$ C NMR (175 MHz, DMSO-d 6 )

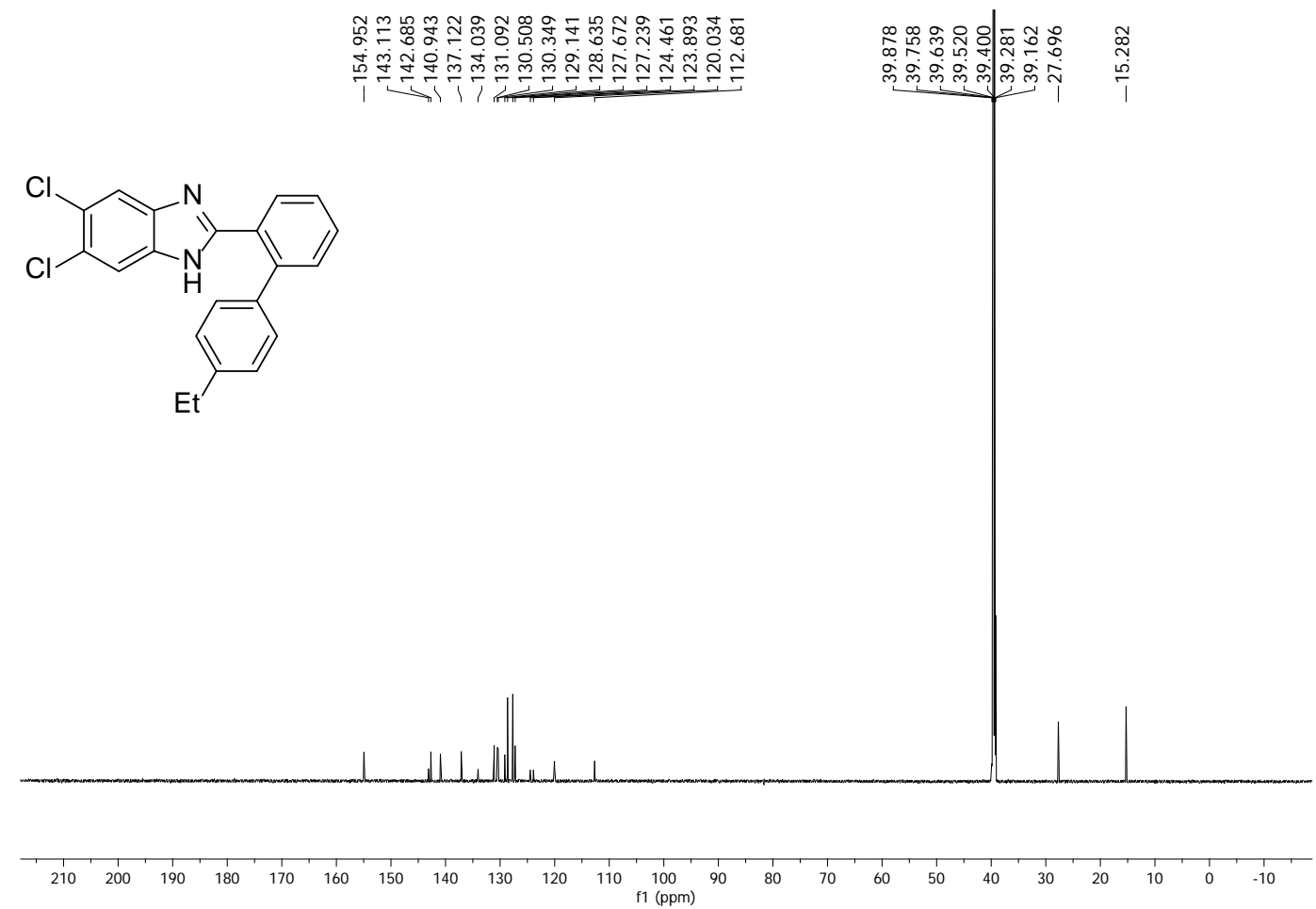

Figure S13. ${ }^{13} \mathrm{C}$ NMR spectrum of 5,6-dichloro-2-(4'-ethyl-[1,1'-biphenyl]-2-yl)-1Hbenzo[d]imidazole (1f). 
${ }^{1}$ H NMR (700 MHz, DMSO-d $)_{6}$

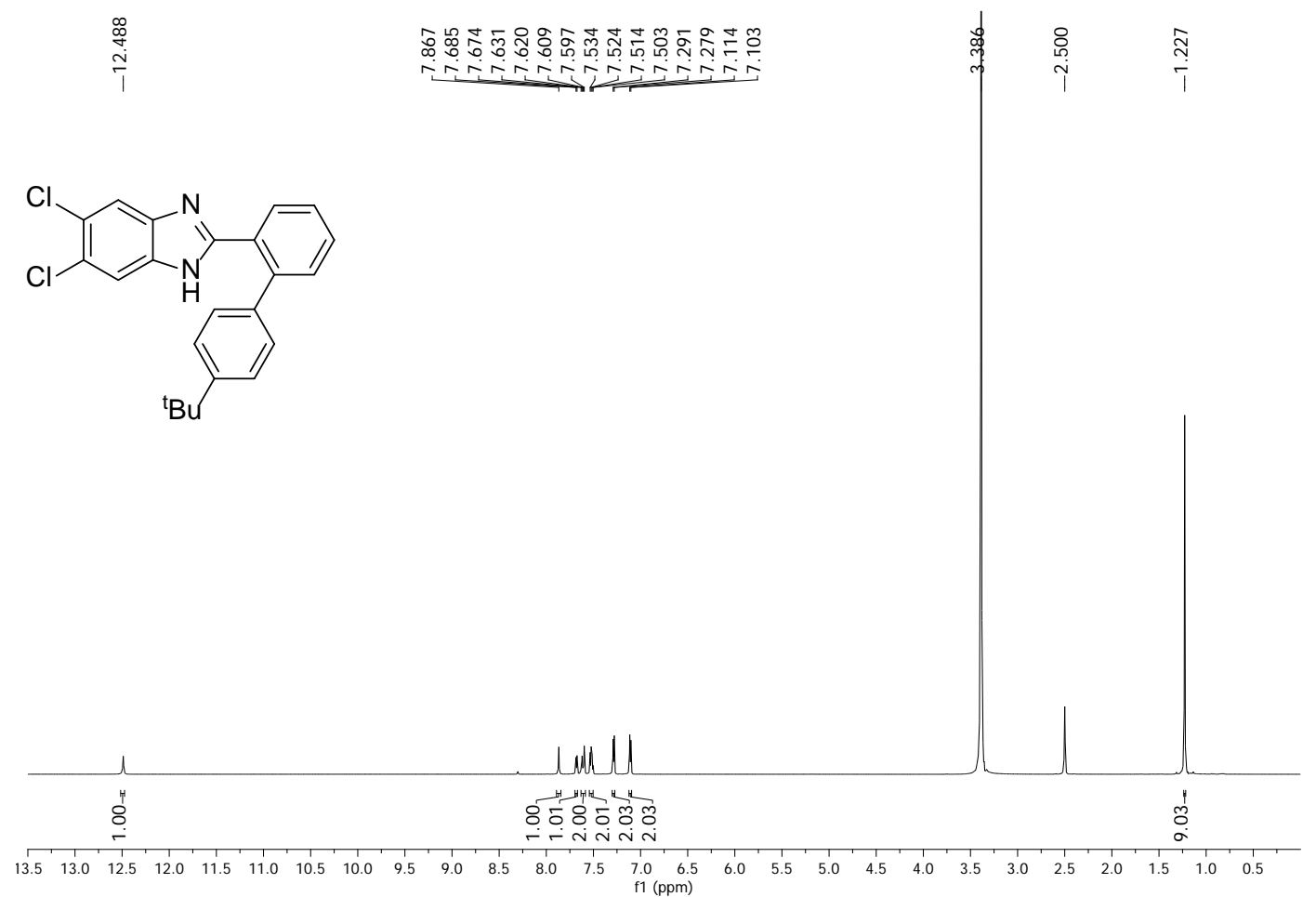

Figure S14. ${ }^{1} \mathrm{H}$ NMR spectrum of 2-(4'-(Tert-butyl)-[1,1'-biphenyl]-2-yl)-5,6-dichloro-1Hbenzo[d]imidazole (19).

${ }^{13}$ C NMR (175 MHz, DMSO-d ${ }_{6}$ )

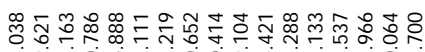

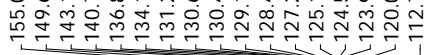

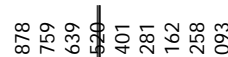

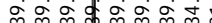

(c)

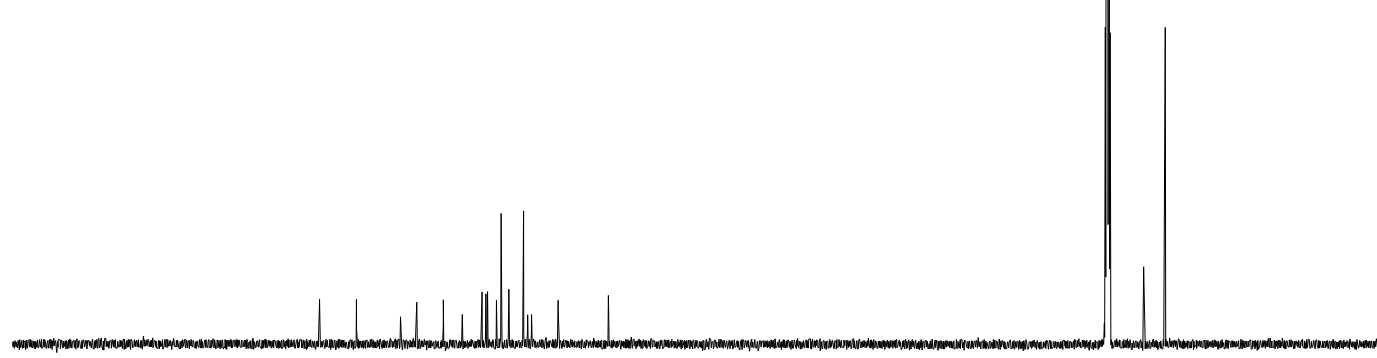

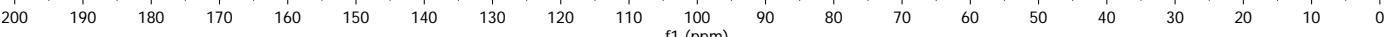

Figure S15. ${ }^{13} \mathrm{C}$ NMR spectrum of 2-(4'-(Tert-butyl)-[1,1'-biphenyl]-2-yl)-5,6-dichloro-1Hbenzo[d]imidazole (19). 
${ }^{1}$ H NMR (700 MHz, DMSO-d $)_{6}$

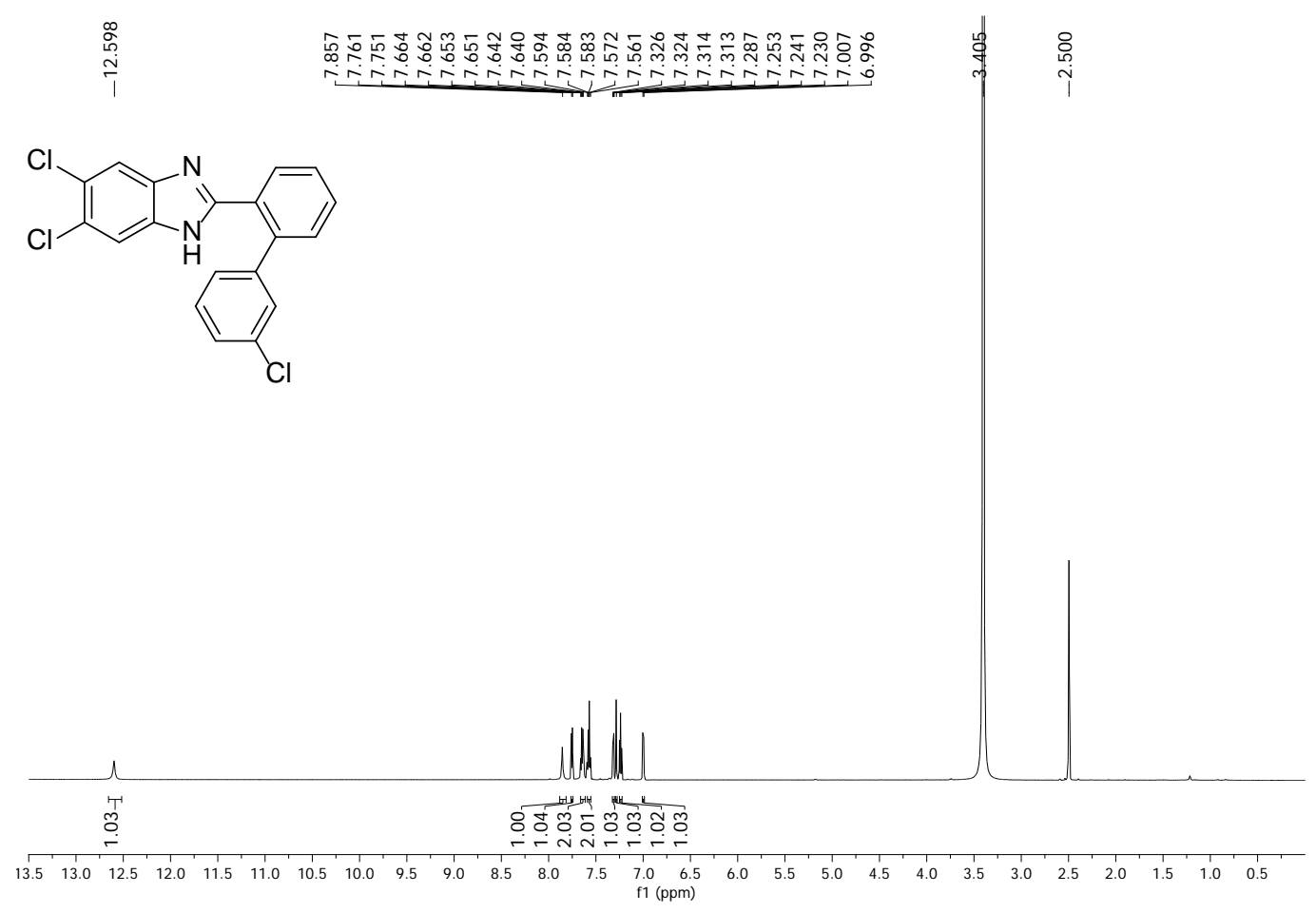

Figure S16. ${ }^{1} \mathrm{H}$ NMR spectrum of 5,6-Dichloro-2-(3'-chloro-[1,1'-biphenyl]-2-yl)-1Hbenzo[d]imidazole (1h).

${ }^{13}$ C NMR (175 MHz, DMSO-d $\left.{ }_{6}\right)$

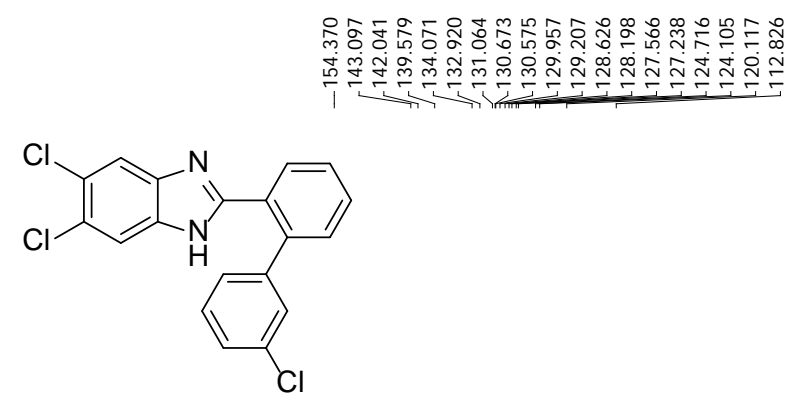

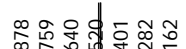

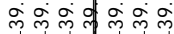

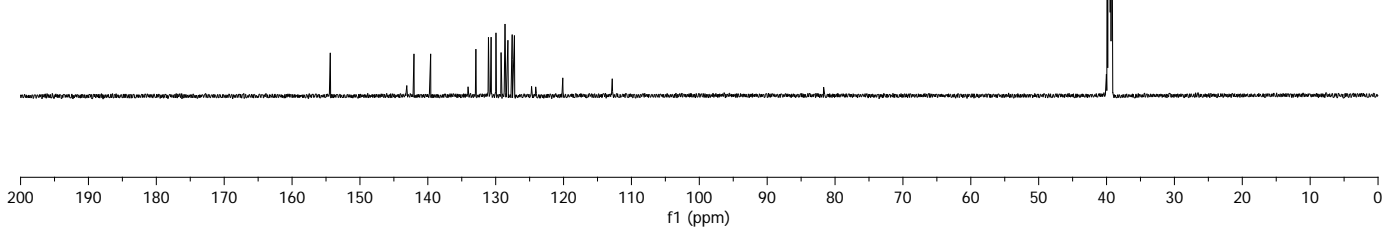

Figure S17. ${ }^{13} \mathrm{C}$ NMR spectrum of 5,6-Dichloro-2-(3'-chloro-[1,1'-biphenyl]-2-yl)-1Hbenzo[d]imidazole (1h). 
${ }^{1}$ H NMR (400 MHz, DMSO-d $)_{6}$

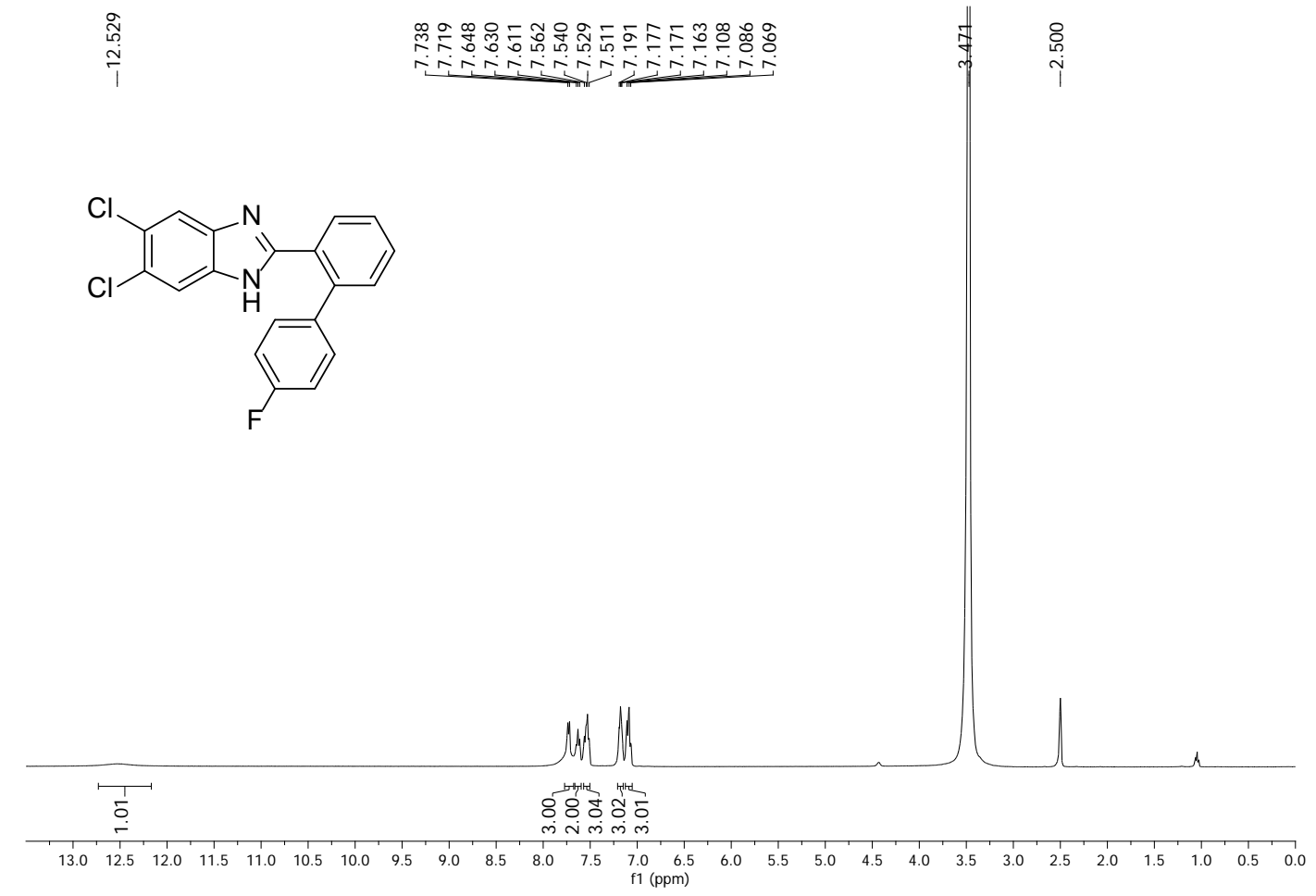

Figure S18. ${ }^{1} \mathrm{H}$ NMR spectrum of 5,6-dichloro-2-(4'-fluoro-[1,1'-biphenyl]-2-yl)-1Hbenzo[d]imidazole (1i)

${ }^{13}$ C NMR (100 MHz, DMSO-d ${ }_{6}$ )

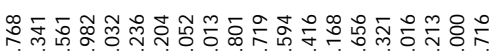

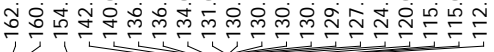<smiles>Fc1ccc(-c2ccccc2-c2nc3cc(Cl)c(Cl)cc3[nH]2)cc1</smiles>

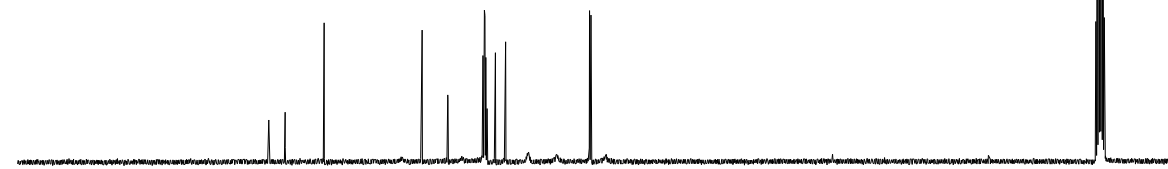

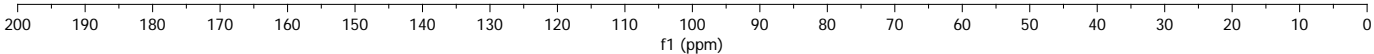

Figure S19. ${ }^{13} \mathrm{C}$ NMR spectrum of 5,6-dichloro-2-(4'-fluoro-[1,1'-biphenyl]-2-yl)-1Hbenzo[d]imidazole (1i) 
${ }^{1}$ H NMR (700 MHz, DMSO-d ) $^{2}$

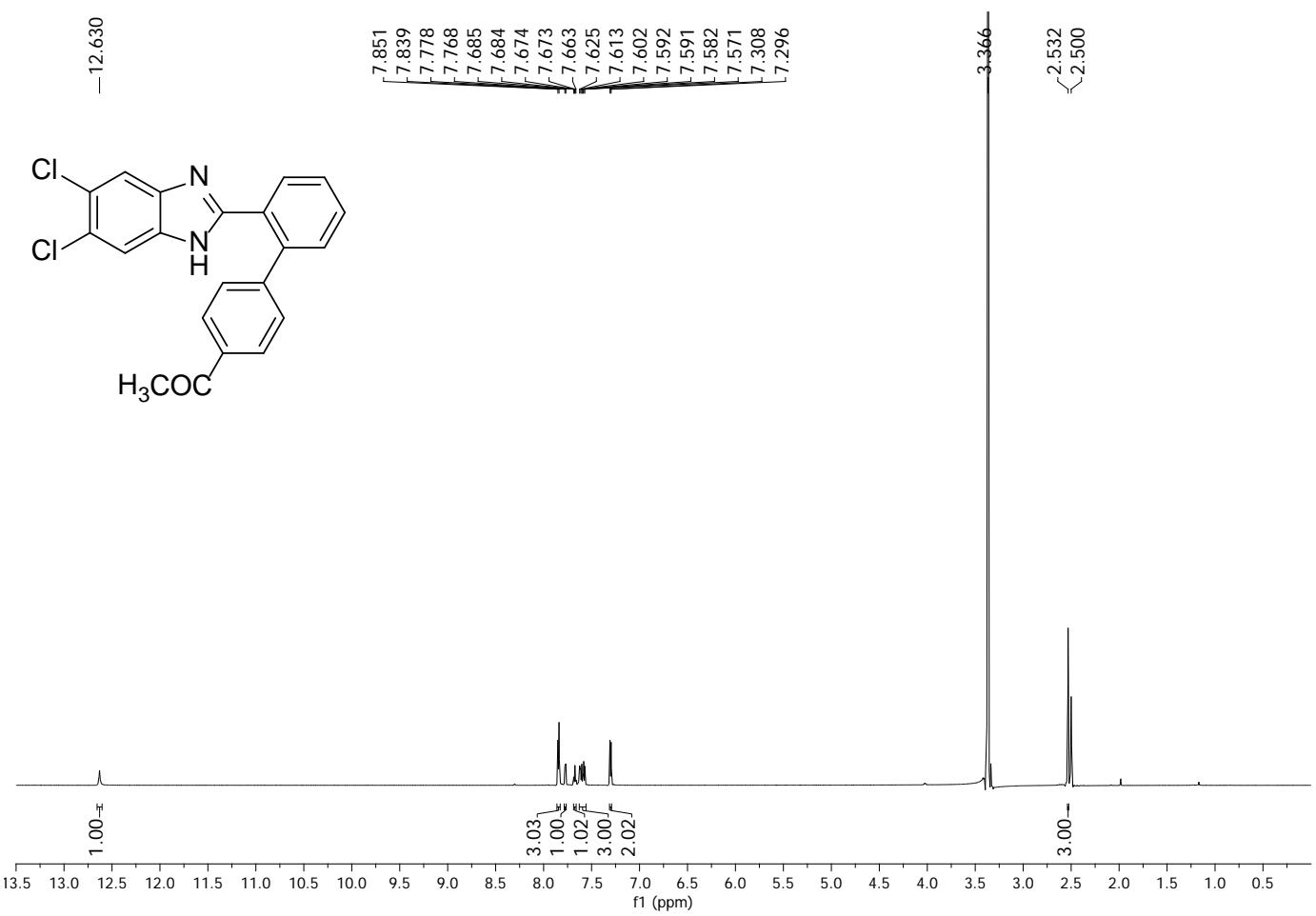

Figure S20. ${ }^{1} \mathrm{H}$ NMR spectrum of 1-(2'-(5,6-Dichloro-1H-benzo[d]imidazol-2-yl)-[1,1'biphenyl]-4-yl)ethan-1-one (1j).

${ }^{13}$ C NMR (175 MHz, DMSO-d $\left.{ }_{6}\right)$
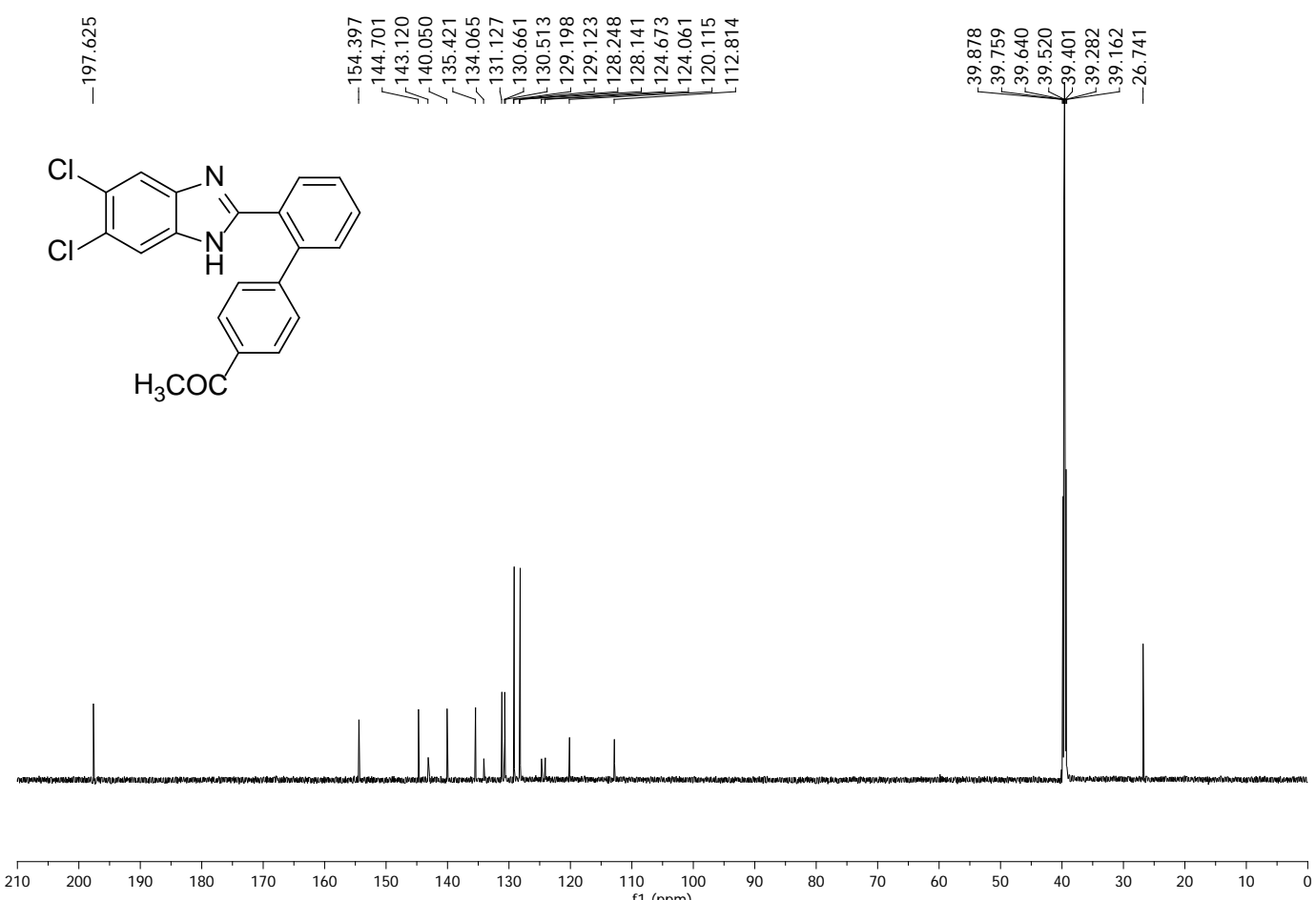

Figure S21. ${ }^{13} \mathrm{C}$ NMR spectrum of 1-(2'-(5,6-Dichloro-1H-benzo[d]imidazol-2-yl)-[1,1'biphenyl]-4-yl)ethan-1-one (1j). 
${ }^{1}$ H NMR (400 MHz, DMSO-d ) $^{2}$

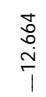

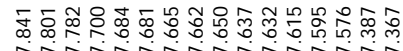

$\stackrel{m}{m} \stackrel{\substack{n \\ j}}{i}$<smiles>FC(F)(F)c1ccc(-c2ccccc2-c2nc3cc(Cl)c(Cl)cc3[nH]2)cc1</smiles>

Wint

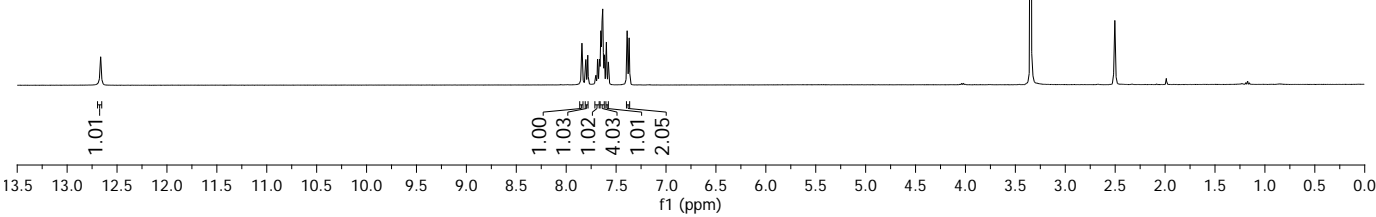

Figure S22. ${ }^{1} \mathrm{H}$ NMR spectrum of 5,6-Dichloro-2-(4'-(trifluoromethyl)-[1,1'-biphenyl]-2-yl)-1Hbenzo[d]imidazole (1k).

${ }^{13}$ C NMR (100 MHz, DMSO-d 6 )

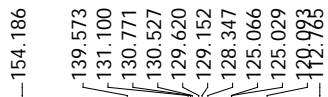

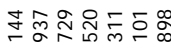

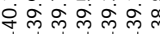<smiles>FC(F)(F)c1ccc(-c2ccccc2-c2nc3cc(Cl)c(Cl)cc3[nH]2)cc1</smiles>

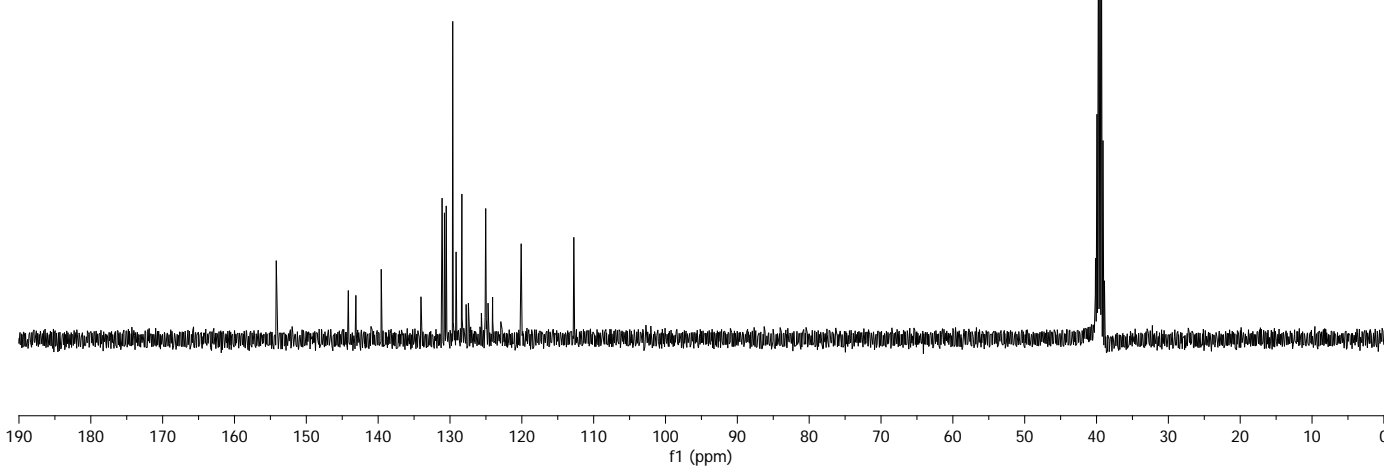

Figure S23. ${ }^{13} \mathrm{C}$ NMR spectrum of 5,6-Dichloro-2-(4'-(trifluoromethyl)-[1,1'-biphenyl]-2-yl)-1Hbenzo[d]imidazole (1k). 
${ }^{1}$ H NMR (700 MHz, DMSO-d $)_{6}$

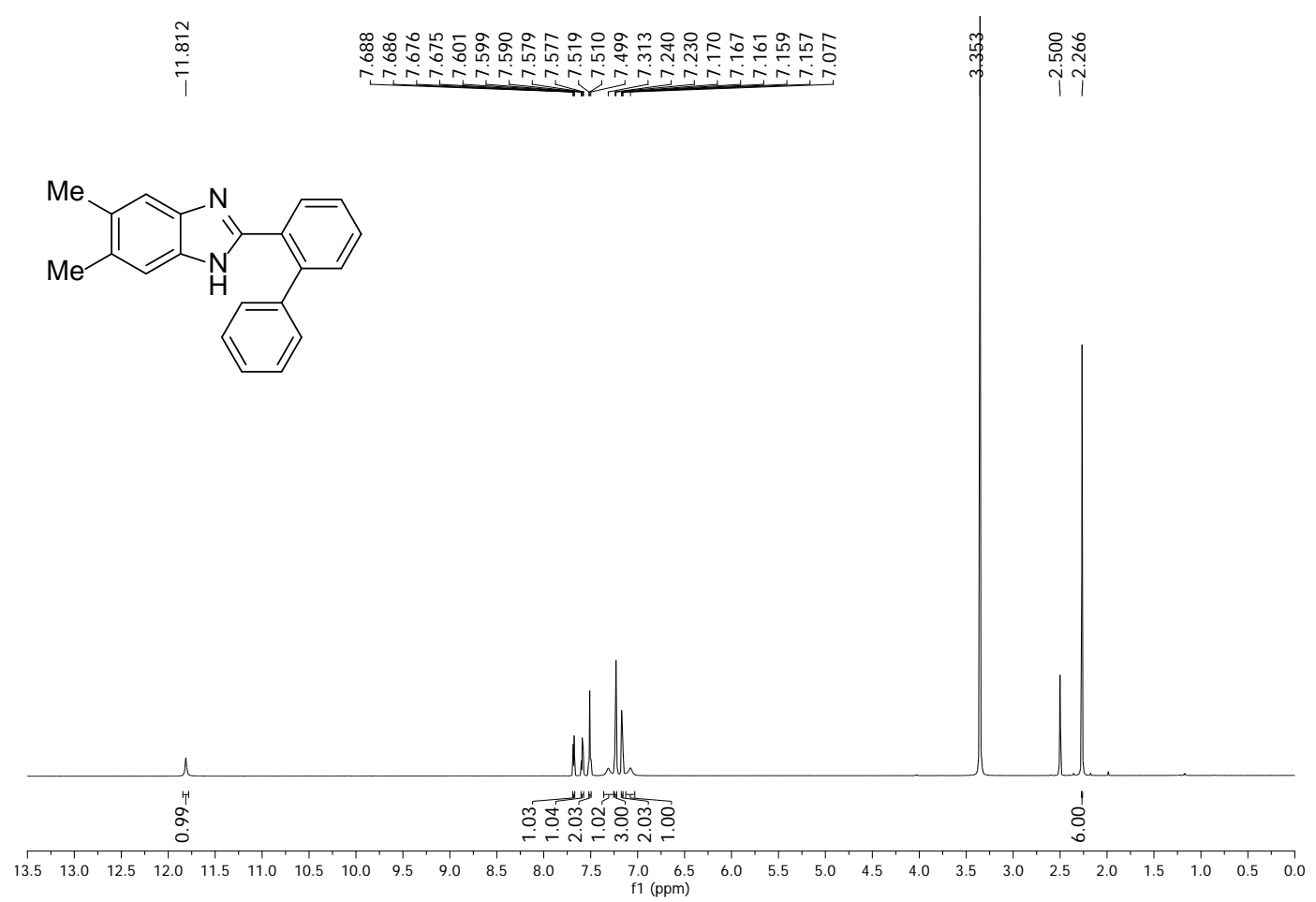

Figure S24. ${ }^{1} \mathrm{H}$ NMR spectrum of 2-([1,1'-Biphenyl]-2-yl)-5,6-dimethyl-1Hbenzo[d]imidazole (11).

${ }^{13}$ C NMR (175 MHz, DMSO-d $)$
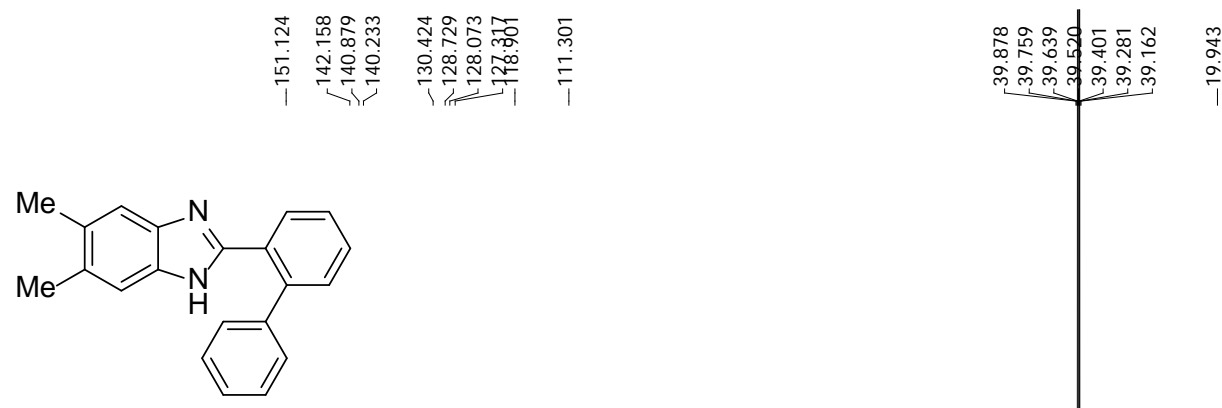

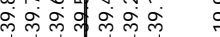

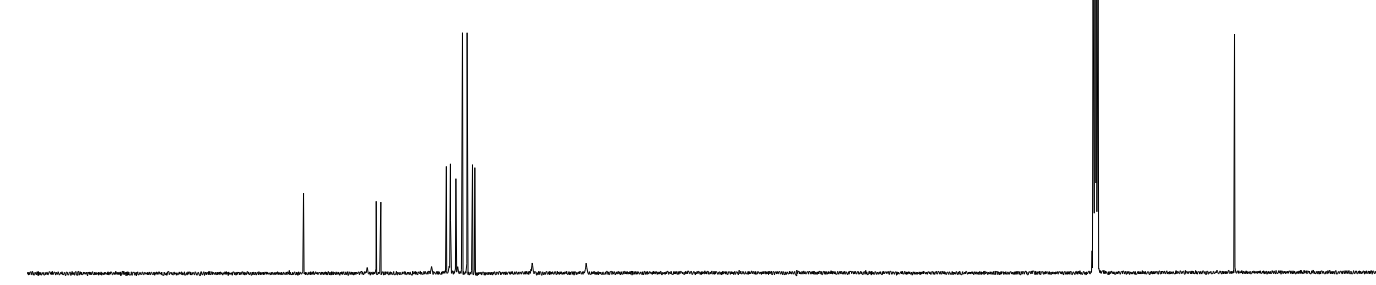

$\begin{array}{llllllllllllllllllll}190 & 180 & 170 & 160 & 150 & 140 & 130 & 120 & 110 & 100 & 90 & 80 & 70 & 60 & 50 & 40 & 30 & 20 & 10 & 0\end{array}$

Figure S25. ${ }^{13} \mathrm{C}$ NMR spectrum of 2-([1,1'-Biphenyl]-2-yl)-5,6-dimethyl-1Hbenzo[d]imidazole (11). 


\section{${ }^{1}$ H NMR (700 MHz, DMSO-d ${ }_{6}$ )}

$\infty$
$\stackrel{2}{2}$
$\stackrel{-1}{1}$

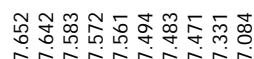

(l)

initicisin

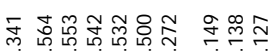
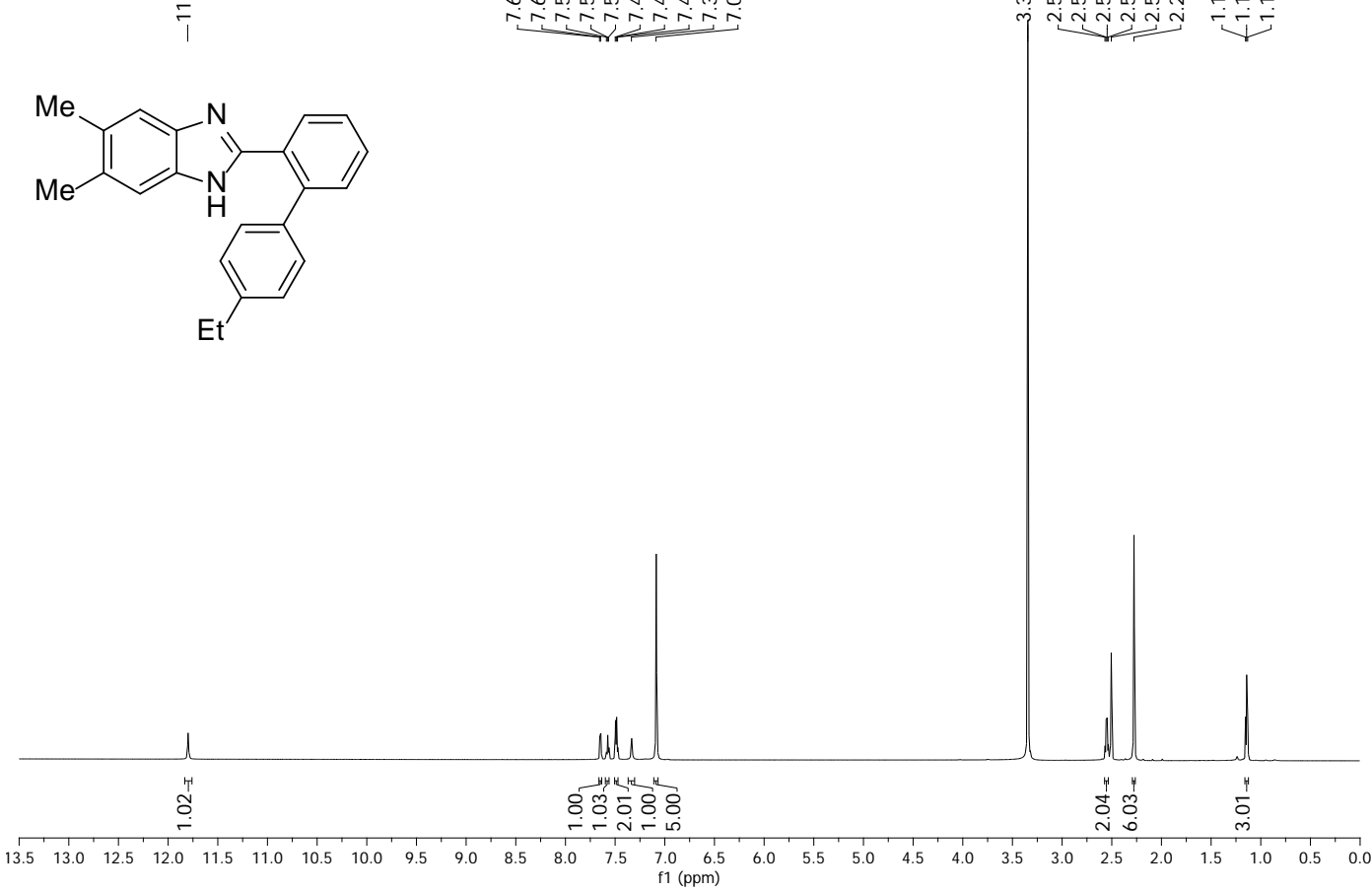

Figure S26. ${ }^{1} \mathrm{H}$ NMR spectrum of 2-(4'-Ethyl-[1,1'-biphenyl]-2-yl)-5,6-dimethyl-1Hbenzo[d]imidazole (1m).

${ }^{13}$ C NMR (175 MHz, DMSO-d ${ }_{6}$ )

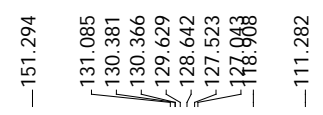<smiles>CCc1ccc(-c2ccccc2-c2nc3cc(C)c(C)cc3[nH]2)cc1</smiles>

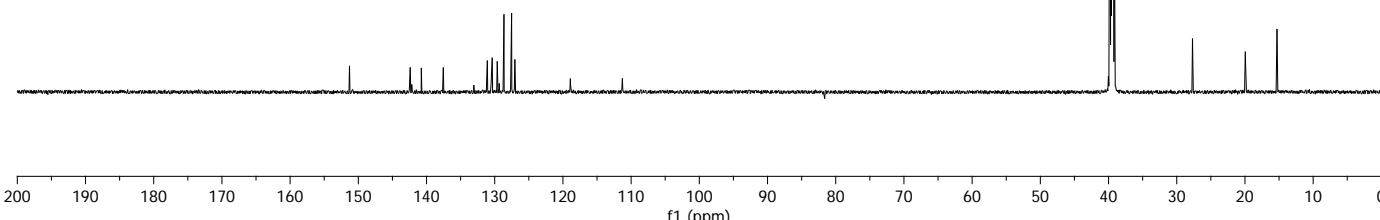

Figure S27. ${ }^{13} \mathrm{C}$ NMR spectrum of 2-(4'-Ethyl-[1,1'-biphenyl]-2-yl)-5,6-dimethyl-1Hbenzo[d]imidazole (1m). 
${ }^{1}$ H NMR (700 MHz, DMSO-d $\left.{ }_{6}\right)$

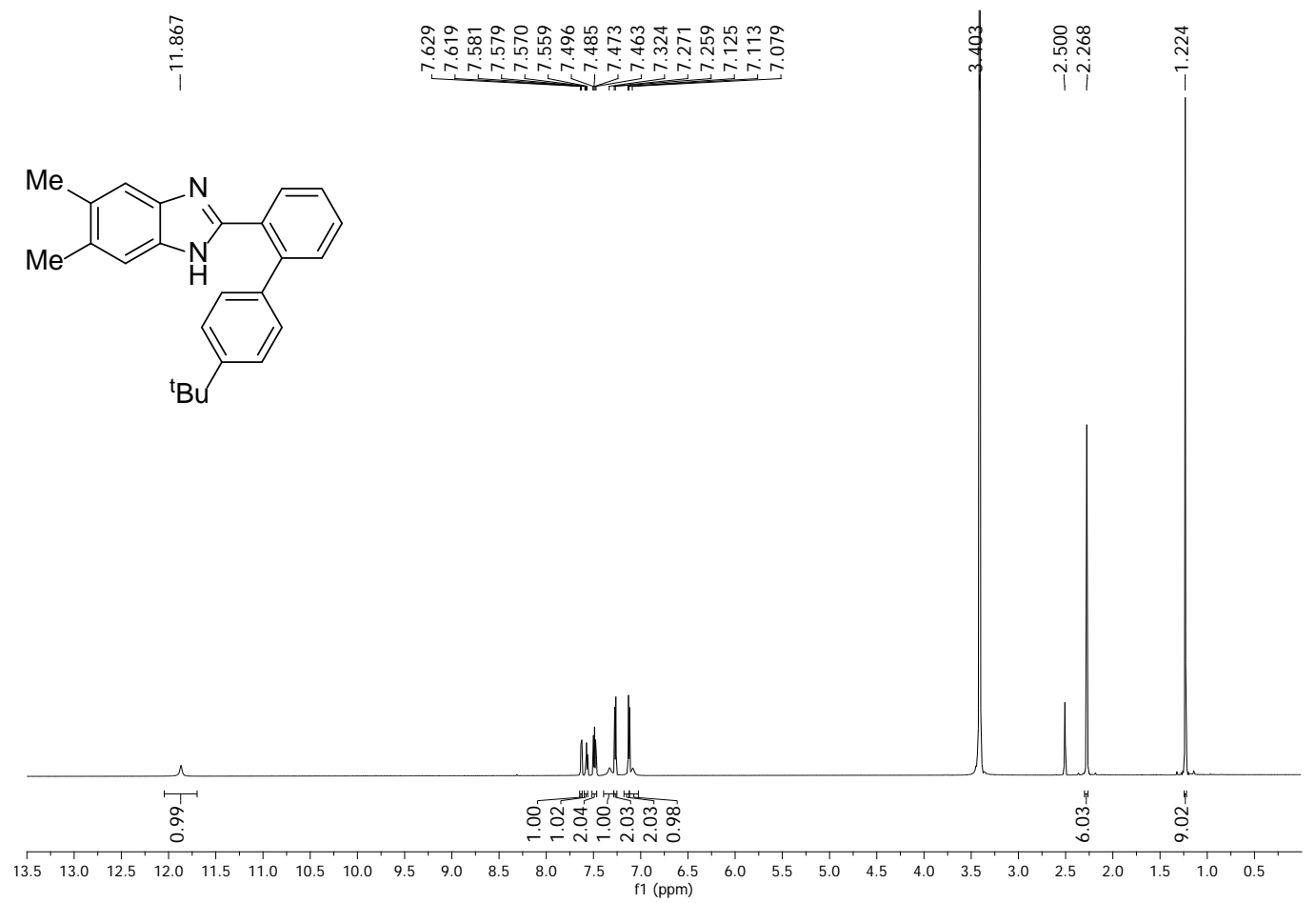

Figure S28. ${ }^{1} \mathrm{H}$ NMR spectrum of 2-(4'-(Tert-butyl)-[1,1'-biphenyl]-2-yl)-5,6-dimethyl-1Hbenzo[d]imidazole (1n).

${ }^{13}$ C NMR (175 MHz, DMSO-d 6 )

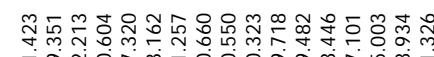

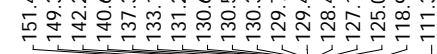
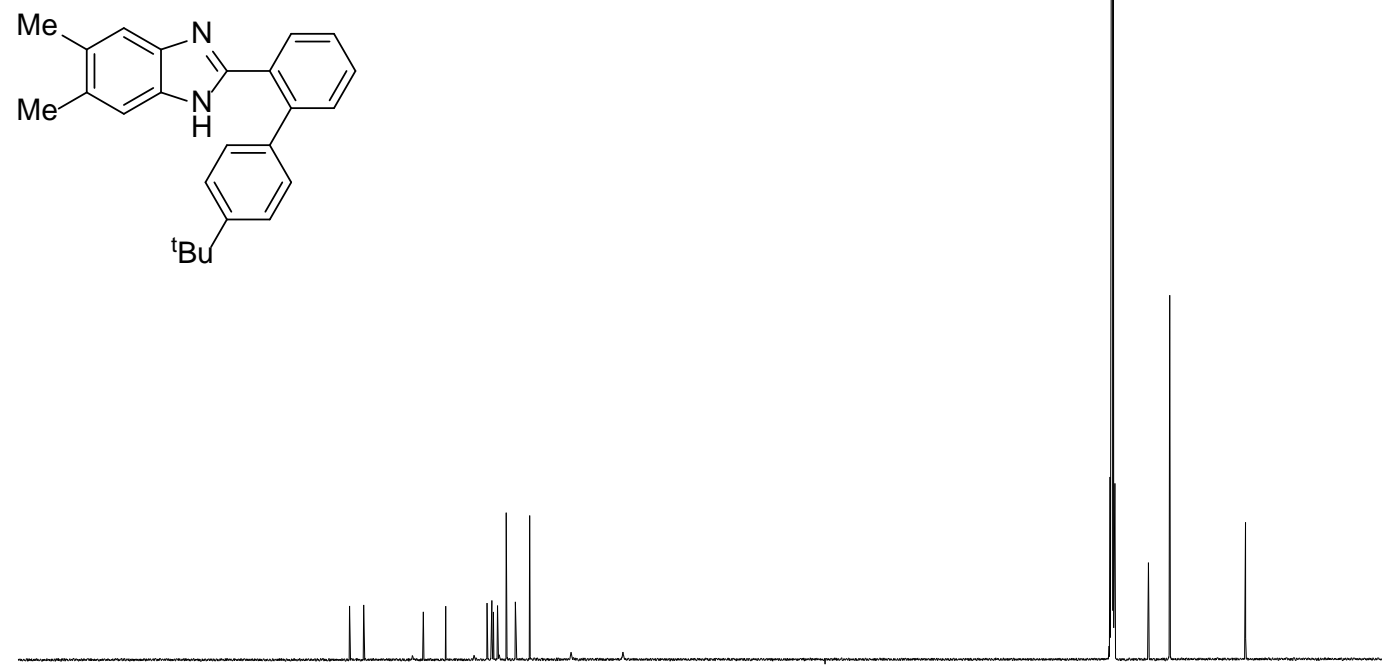

$\begin{array}{lllllllllllllllllllllllllll} & 200 & 190 & 180 & 170 & 160 & 150 & 140 & 130 & 120 & 110 & 100 & 90 & 80 & 70 & 60 & 50 & 40 & 30 & 20 & 10 & 0\end{array}$

Figure S29. ${ }^{13} \mathrm{C}$ NMR spectrum of 2-(4'-(Tert-butyl)-[1,1'-biphenyl]-2-yl)-5,6-dimethyl-1Hbenzo[d]imidazole (1n). 


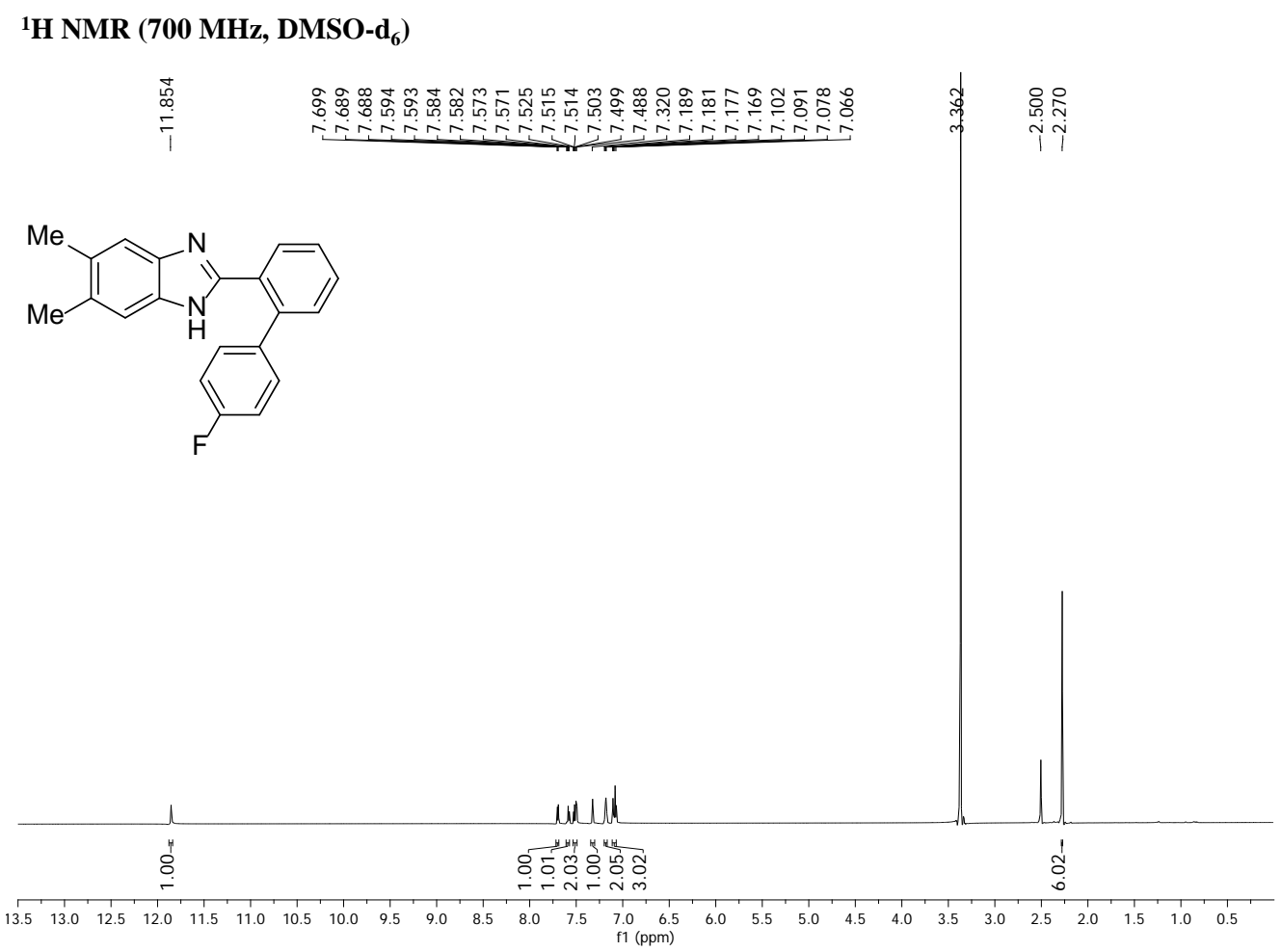

Figure S30. ${ }^{1} \mathrm{H}$ NMR spectrum of 2-(4'-Fluoro-[1,1'-biphenyl]-2-yl)-5,6-dimethyl-1Hbenzo[d]imidazole (10).

${ }^{13}$ C NMR (175 MHz, DMSO-d $\left.{ }_{6}\right)$

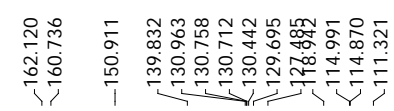

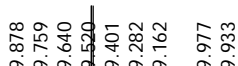

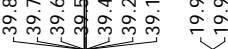
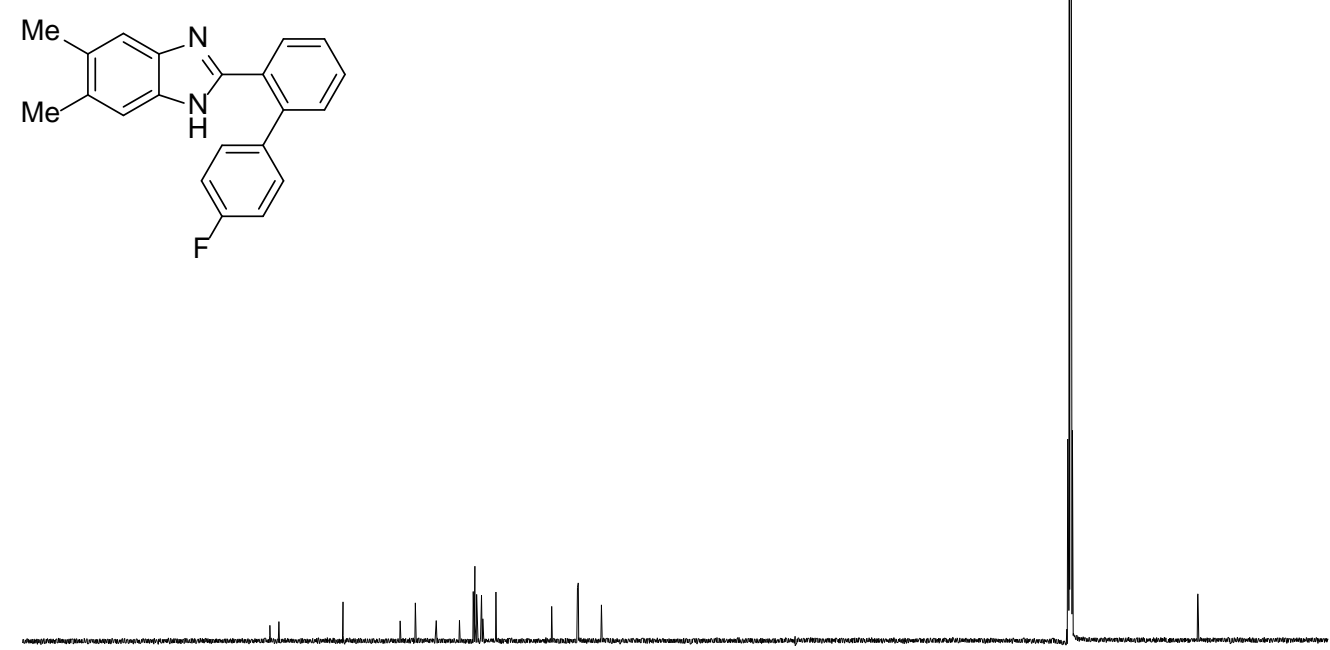

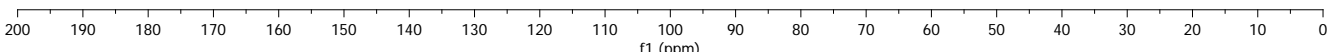

Figure S31. ${ }^{13} \mathrm{C}$ NMR spectrum of 2-(4'-Fluoro-[1,1'-biphenyl]-2-yl)-5,6-dimethyl-1Hbenzo[d]imidazole (10). 


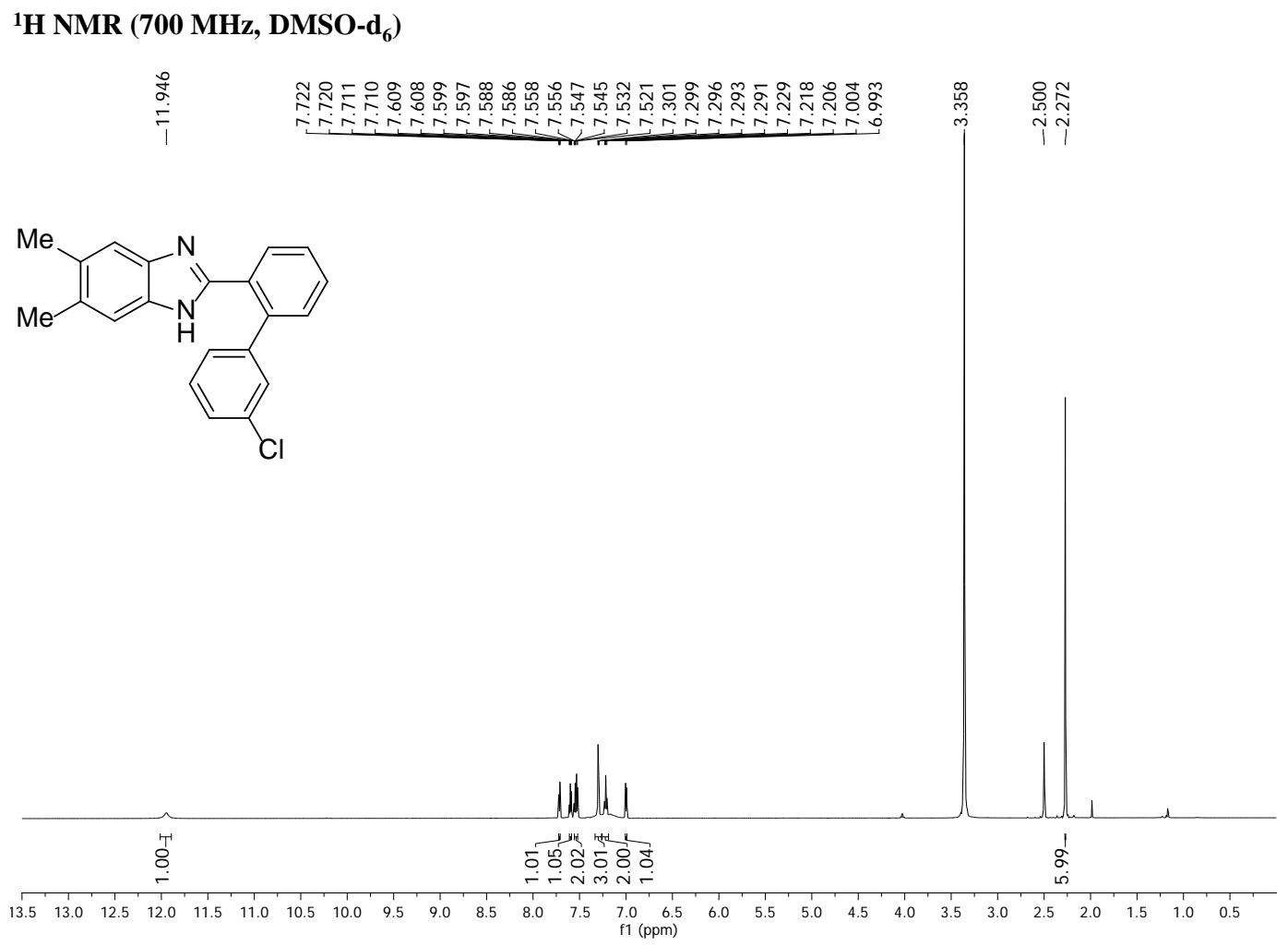

Figure S32. ${ }^{1} \mathrm{H}$ NMR spectrum of 2-(3'-Chloro-[1,1'-biphenyl]-2-yl)-5,6-dimethyl-1Hbenzo[d]imidazole (1p).

${ }^{13}$ C NMR (175 MHz, DMSO-d $)$
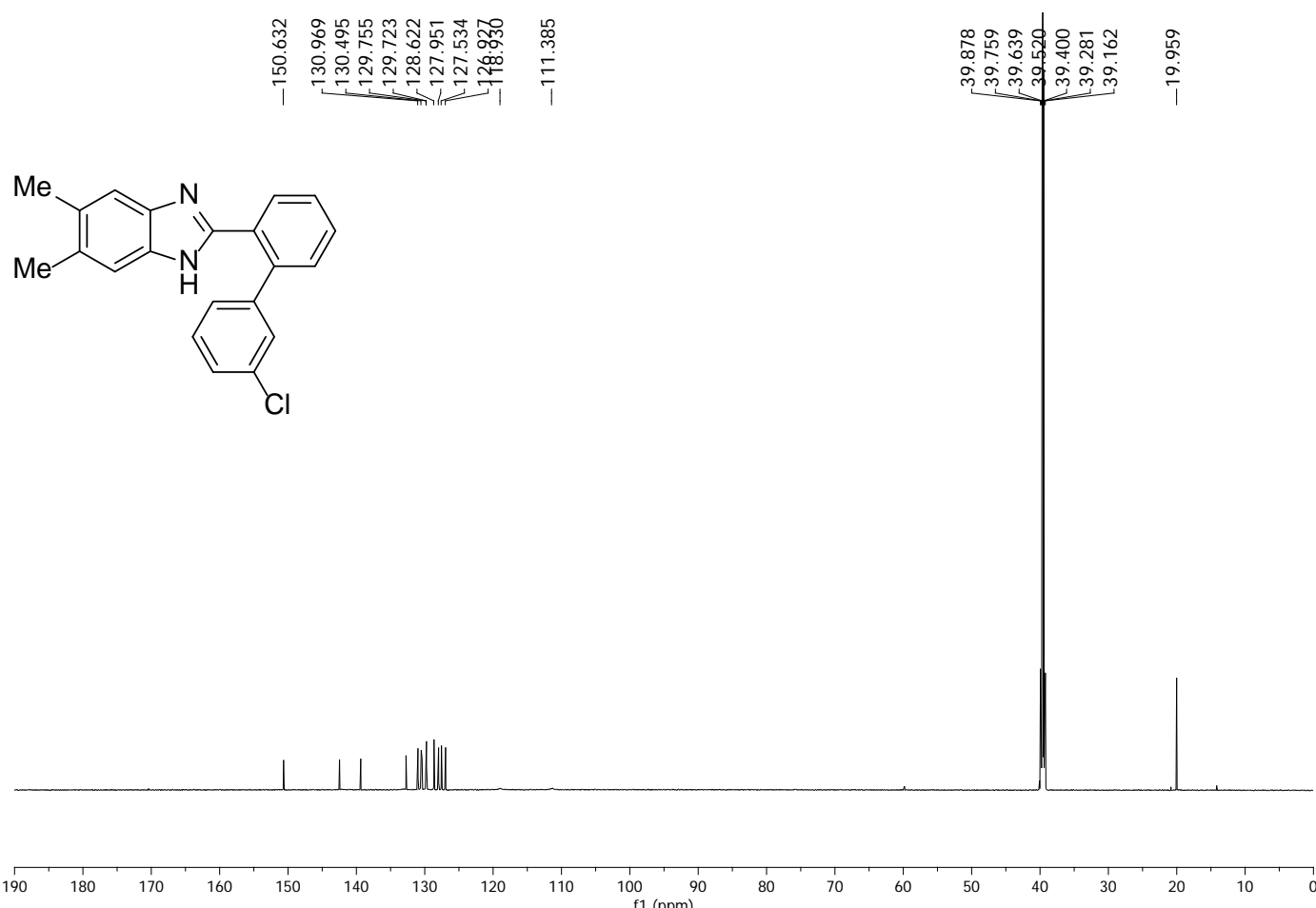

Figure S33. ${ }^{13} \mathrm{C}$ NMR spectrum of 2-(3'-Chloro-[1,1'-biphenyl]-2-yl)-5,6-dimethyl-1Hbenzo[d]imidazole (1p). 
${ }^{1}$ H NMR (700 MHz, DMSO-d $\left.{ }_{6}\right)$

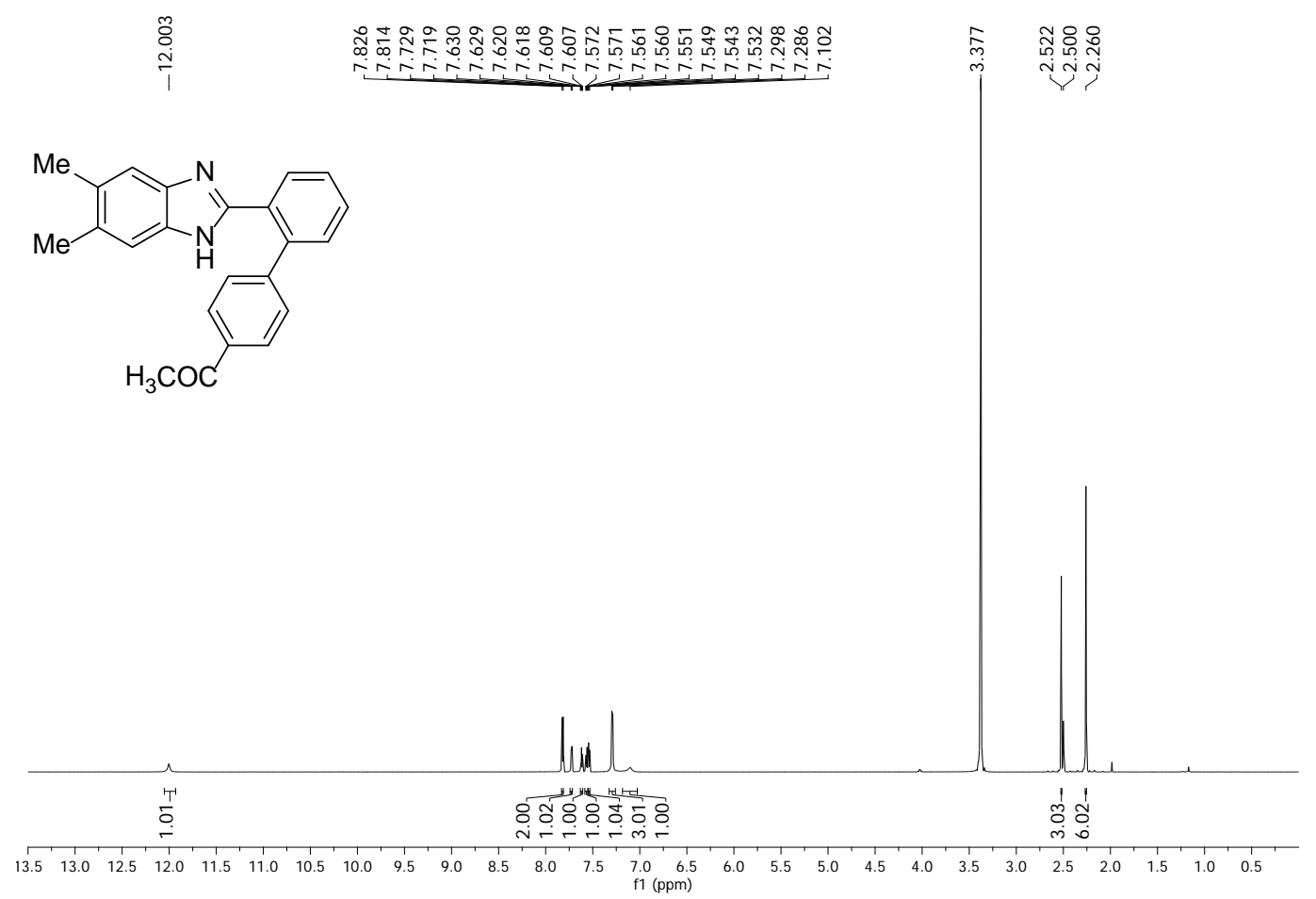

Figure S34. ${ }^{1} \mathrm{H}$ NMR spectrum of 1-(2'-(5,6-Dimethyl-1H-benzo[d]imidazol-2-yl)-[1,1'biphenyl]-4-yl)ethan-1-one (1q).

${ }^{13}$ C NMR (175 MHz, DMSO-d 6 )
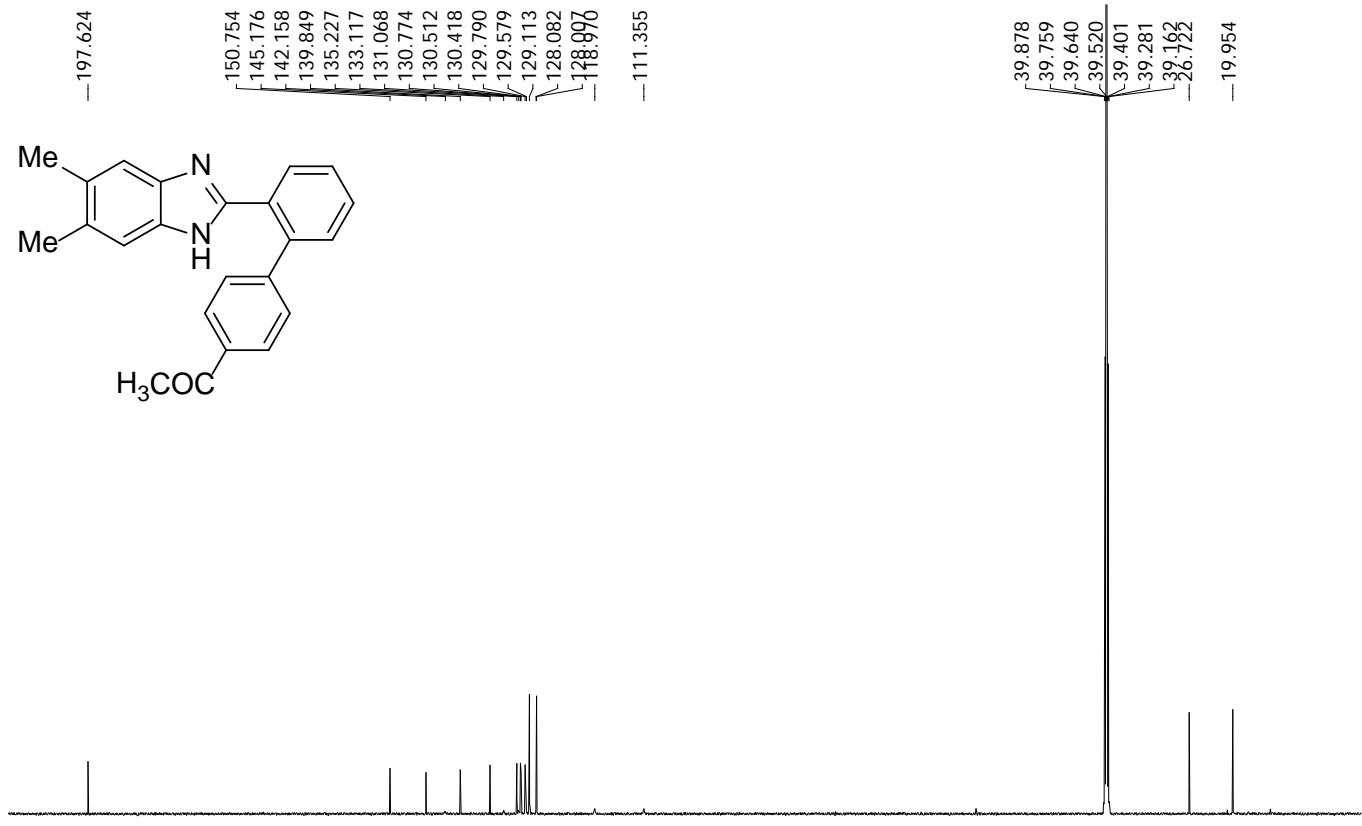

\begin{tabular}{llllllllllllllllllllllll}
\hline 210 & 200 & 190 & 180 & 170 & 160 & 150 & 140 & 130 & 120 & 110 & 100 & 90 & 10 & 70 & 60 & 50 & 40 & 30 & 20 & 10 & 0 \\
\hline 1
\end{tabular}

Figure S35. ${ }^{13} \mathrm{C}$ NMR spectrum of 1-(2'-(5,6-Dimethyl-1H-benzo[d]imidazol-2-yl)-[1,1'biphenyl]-4-yl)ethan-1-one (1q). 


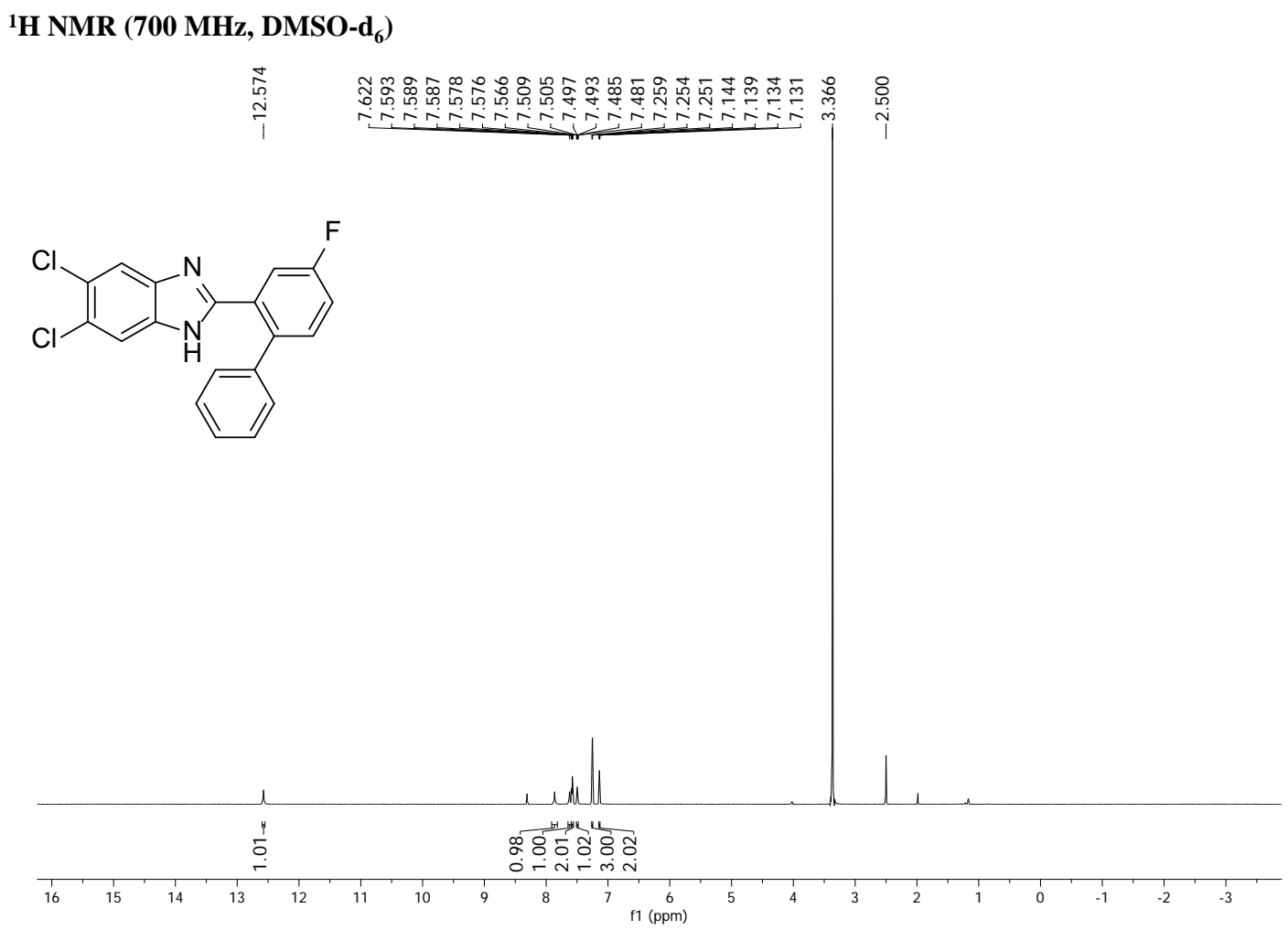

Figure S36. ${ }^{1} \mathrm{H}$ NMR spectrum of 5,6-Dichloro-2-(4-fluoro-[1,1'-biphenyl]-2-yl)-1Hbenzo[d]imidazole (1r).

${ }^{13}$ C NMR (175 MHz, DMSO-d 6 )

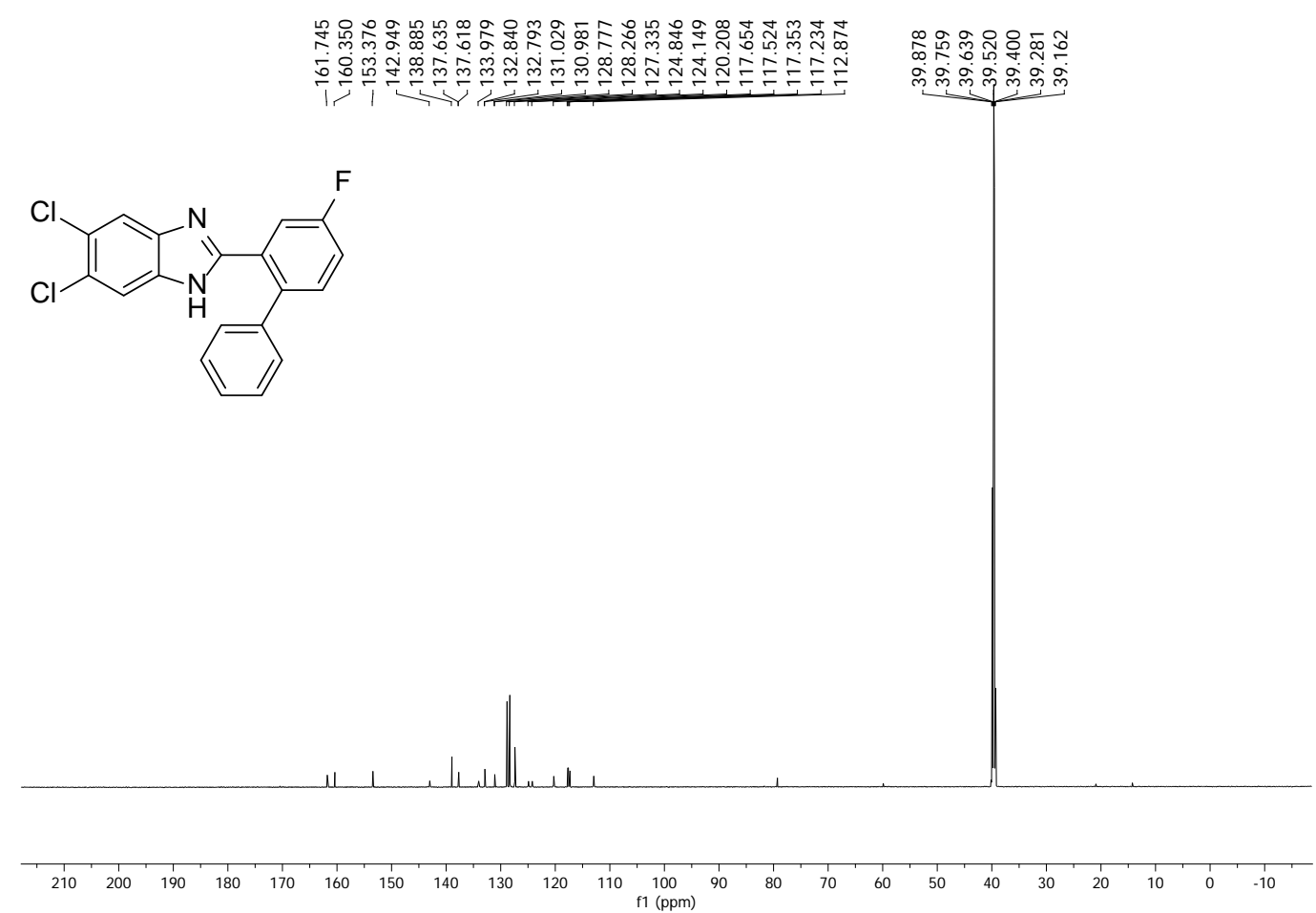

Figure S37. ${ }^{13} \mathrm{C}$ NMR spectrum of 5,6-Dichloro-2-(4-fluoro-[1,1'-biphenyl]-2-yl)-1Hbenzo[d]imidazole (1r). 
${ }^{1}$ H NMR (400 MHz, DMSO-d $\left.{ }_{6}\right)$

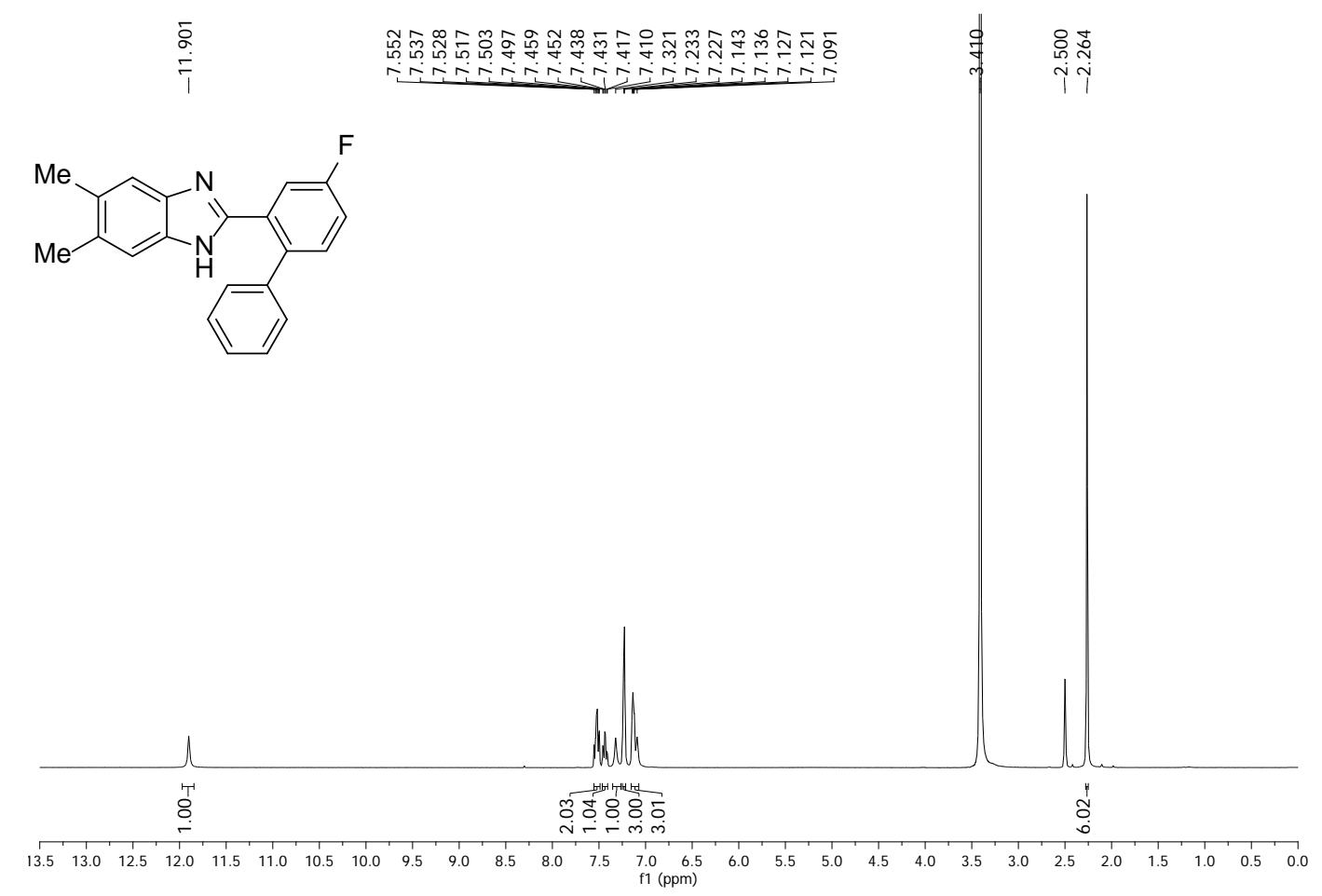

Figure S38. ${ }^{1} \mathrm{H}$ NMR spectrum of 2-(4-Fluoro-[1,1'-biphenyl]-2-yl)-5,6-dimethyl-1Hbenzo[d]imidazole (1s).

${ }^{13}$ C NMR (100 MHz, DMSO-d $)_{6}$

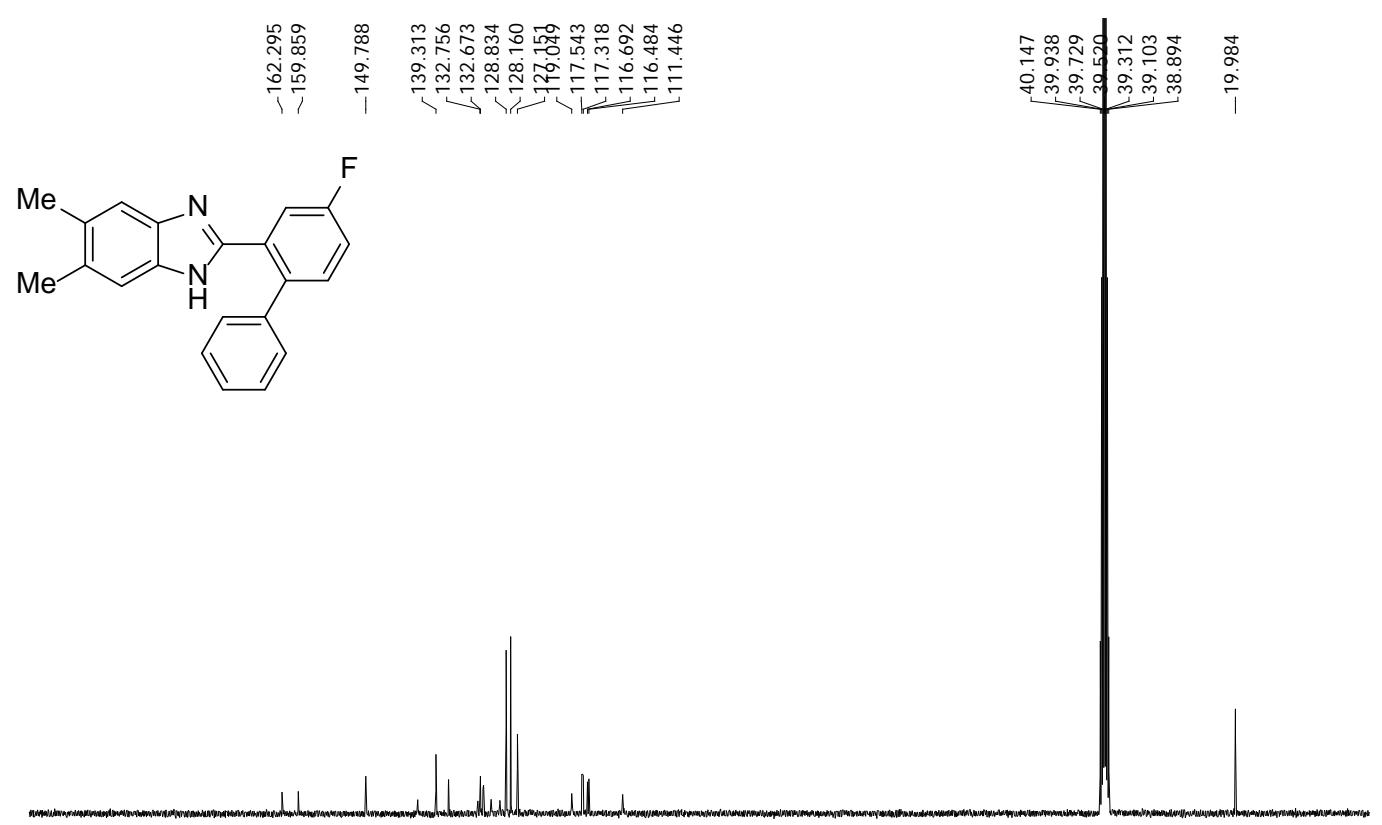

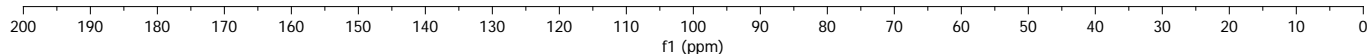

Figure S39. ${ }^{13} \mathrm{C}$ NMR spectrum of 2-(4-Fluoro-[1,1'-biphenyl]-2-yl)-5,6-dimethyl-1Hbenzo[d]imidazole (1s). 
${ }^{1}$ H NMR (700 MHz, DMSO-d $\left.{ }_{6}\right)$

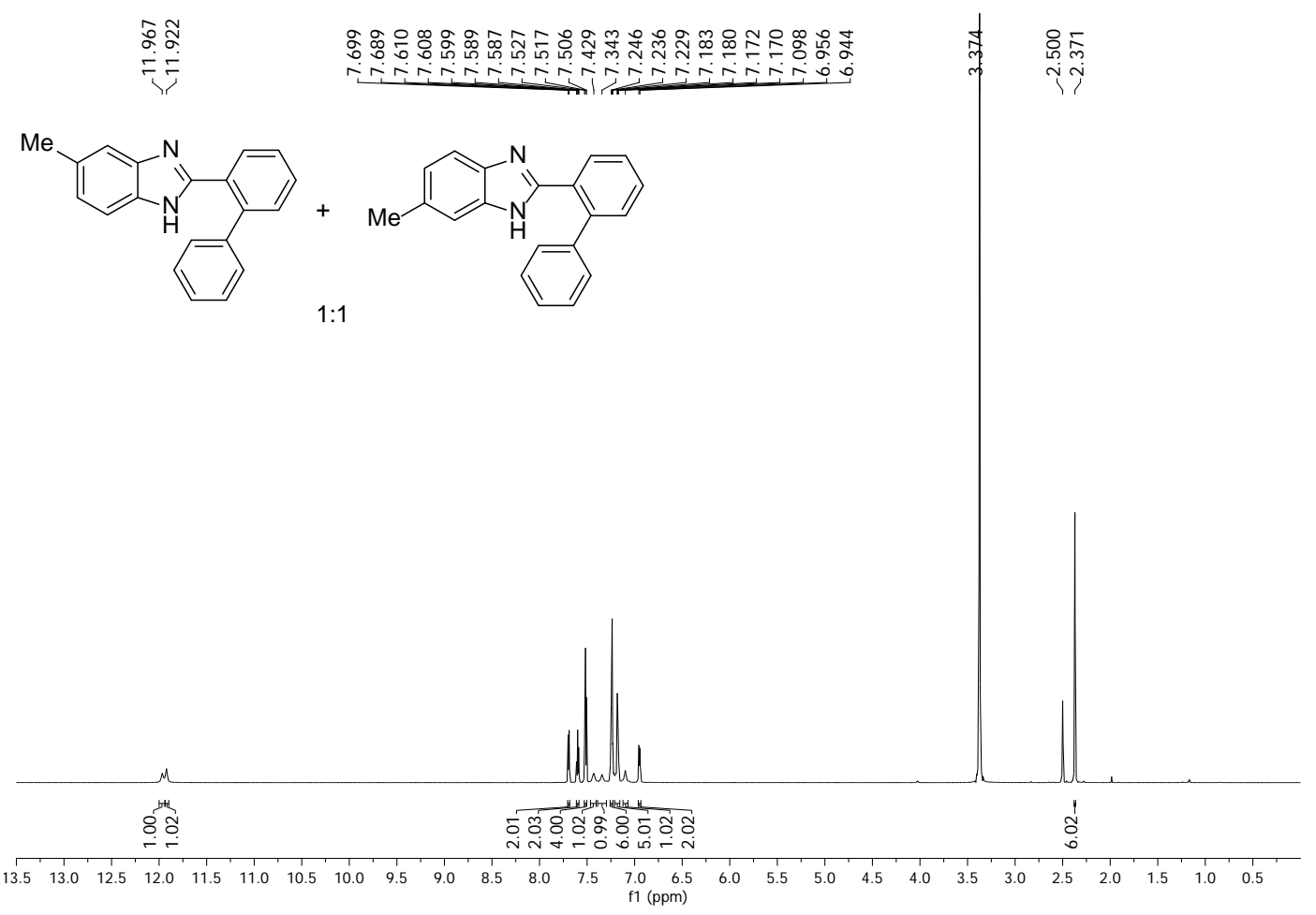

Figure S40. ${ }^{1} \mathrm{H}$ NMR spectrum of 2-([1,1'-Biphenyl]-2-yl)-5-methyl-1H-benzo[d]imidazole and 2-([1,1'-Biphenyl]-2-yl)-6-methyl-1H-benzo[d]imidazole (1a').

${ }^{13} \mathrm{C}$ NMR (175 MHz, DMSO-d (1) $^{2}$
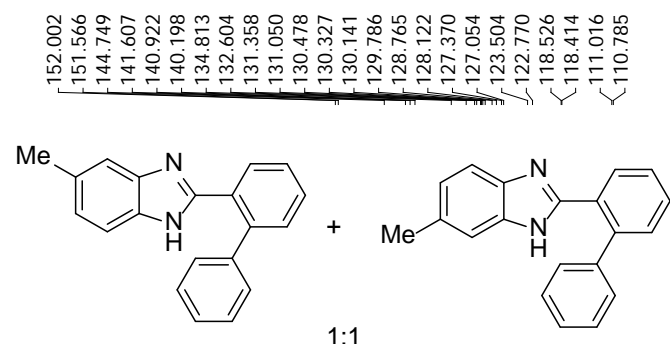

$1: 1$
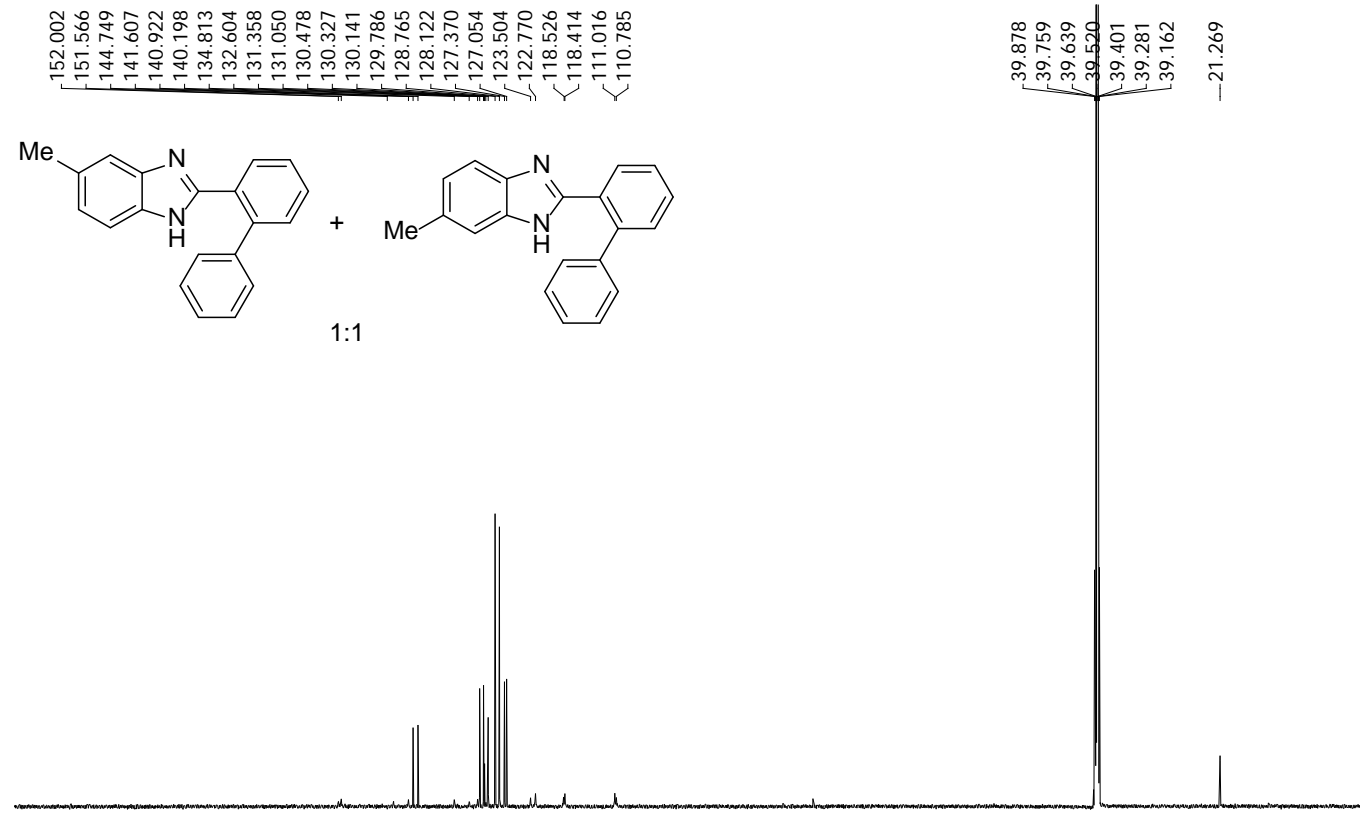

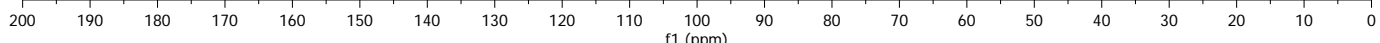

Figure S41. ${ }^{13} \mathrm{C}$ NMR spectrum of 2-([1,1'-Biphenyl]-2-yl)-5-methyl-1H-benzo[d]imidazole and 2-([1,1'-Biphenyl]-2-yl)-6-methyl-1H-benzo[d]imidazole (1a'). 


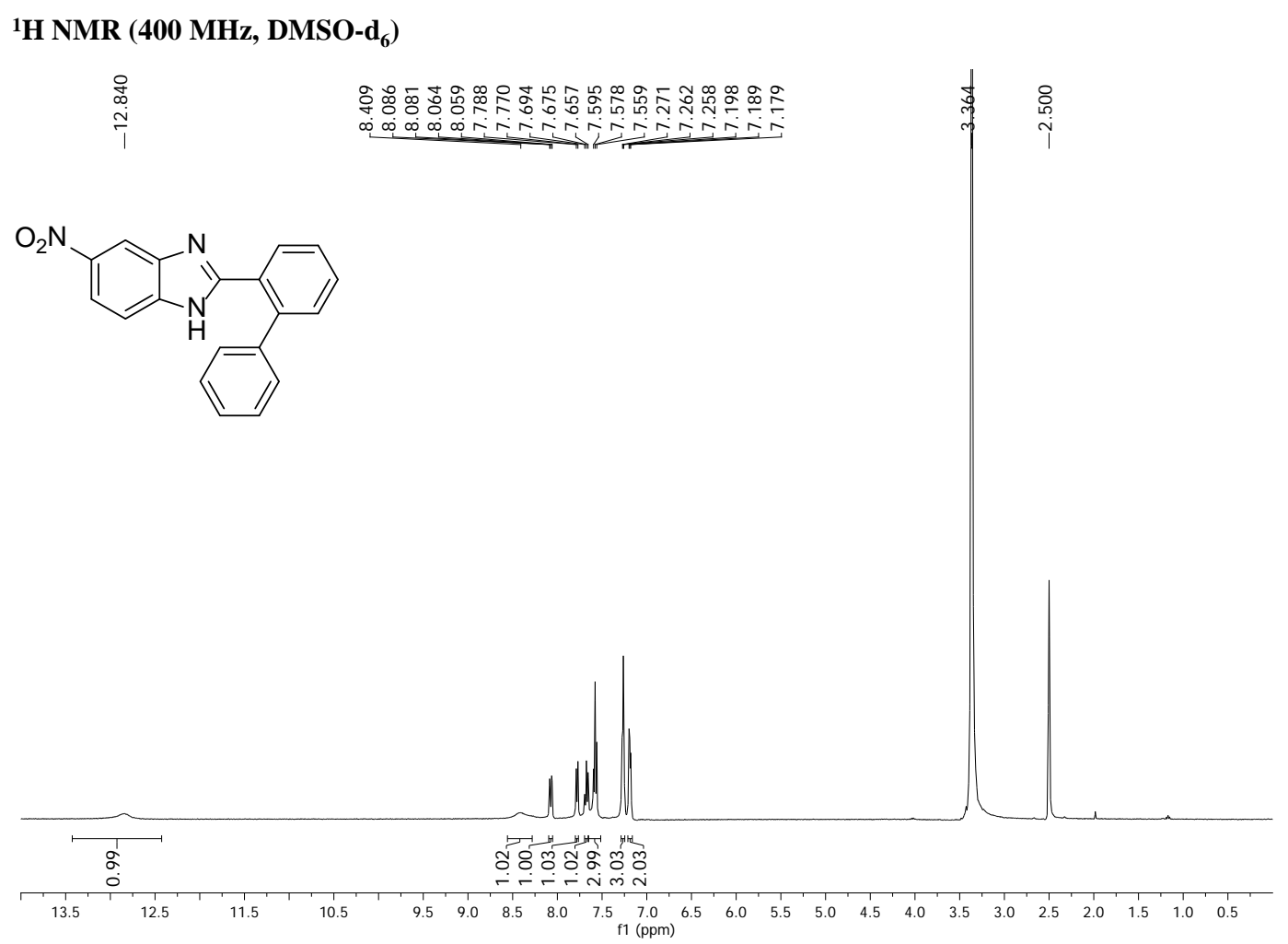

Figure S42. ${ }^{1} \mathrm{H}$ NMR spectrum of 2-([1,1'-Biphenyl]-2-yl)-5-nitro-1H-benzo[d]imidazole (1b').

${ }^{13}$ C NMR (175 MHz, DMSO-d 6 )

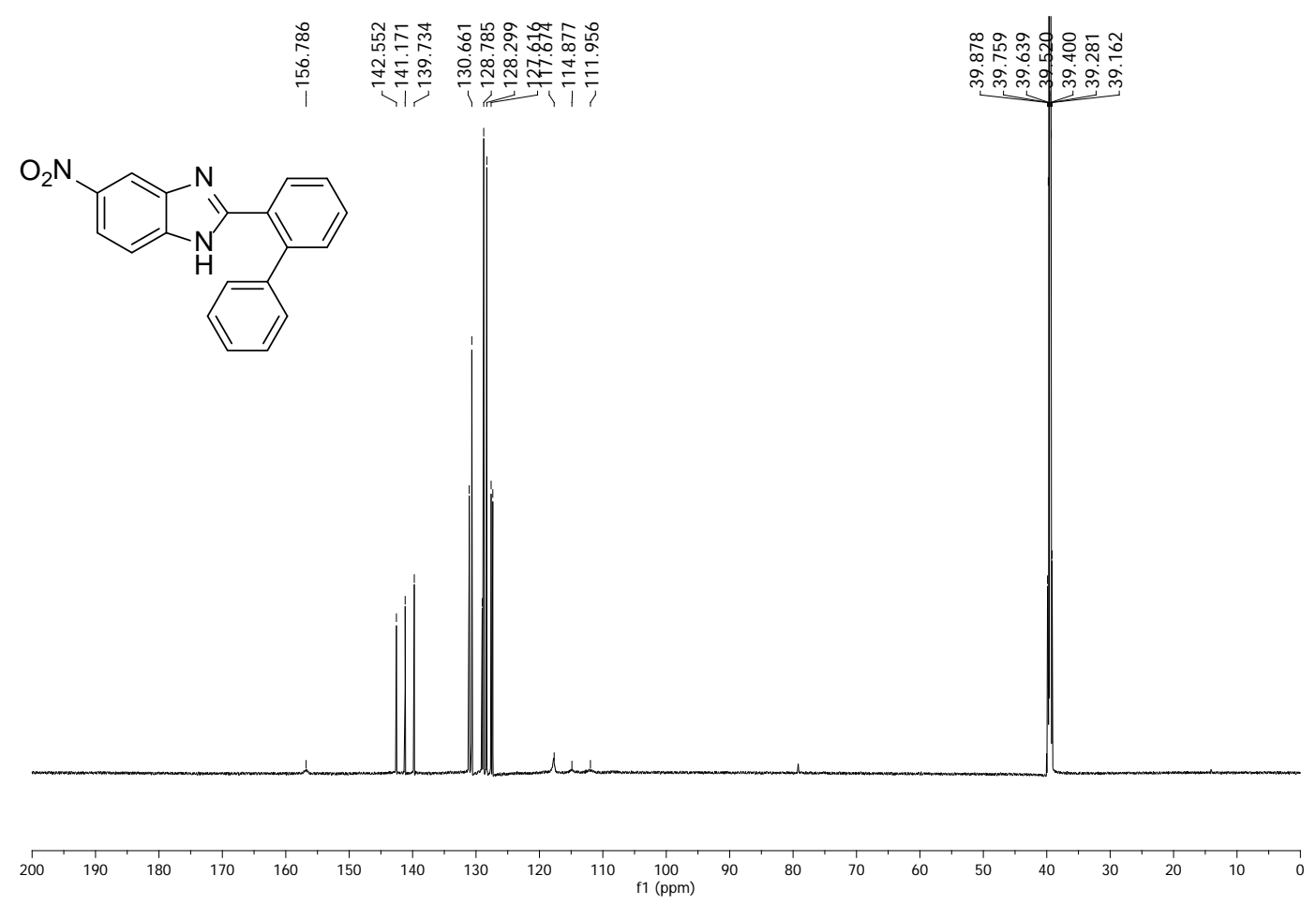

Figure S43. ${ }^{13} \mathrm{C}$ NMR spectrum of 2-([1,1'-Biphenyl]-2-yl)-5-nitro-1H-benzo[d]imidazole (1b') 
${ }^{1}$ H NMR (700 MHz, DMSO-d 6 )

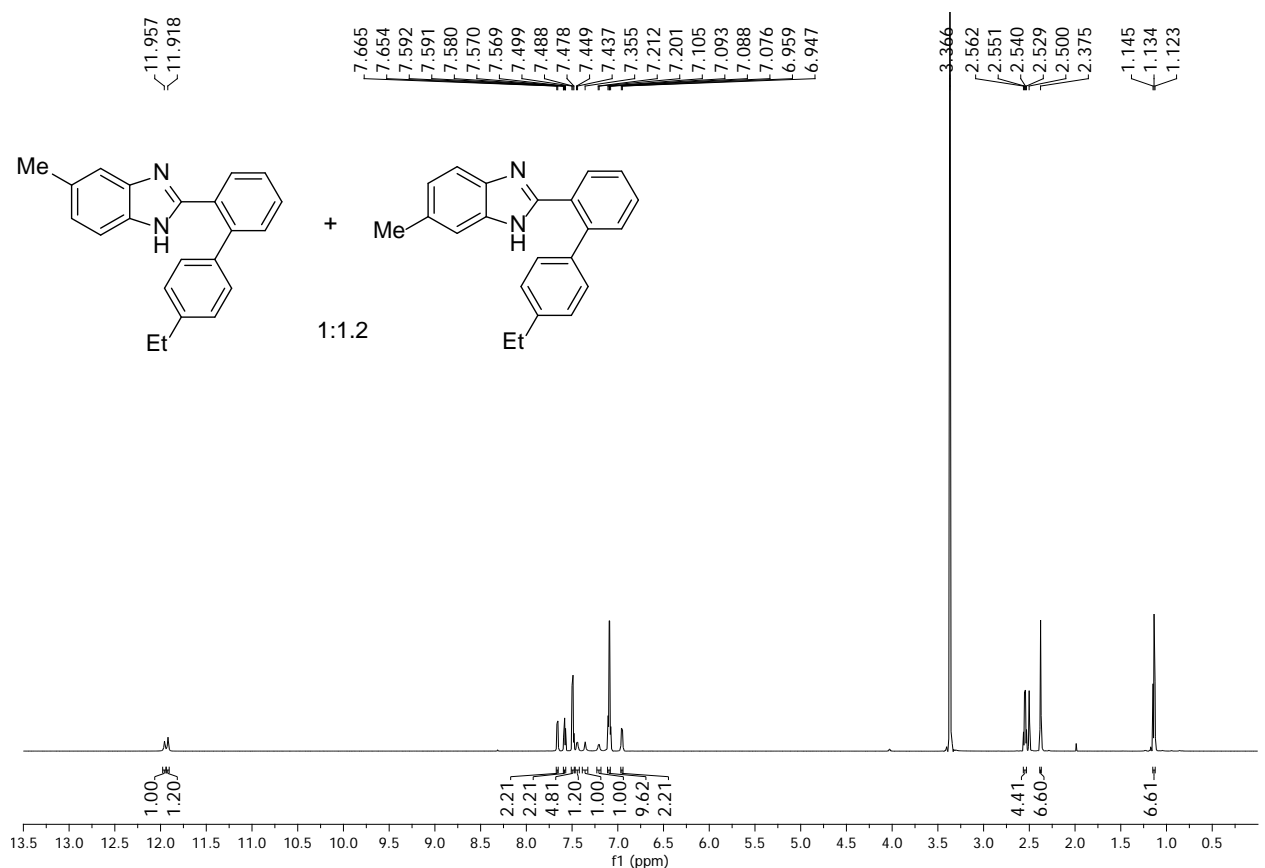

Figure S44. ${ }^{1} \mathrm{H}$ NMR spectrum of 2-(4'-Ethyl-[1,1'-biphenyl]-2-yl)-5-methyl-1Hbenzo[d]imidazole and 2-(4'-Ethyl-[1,1'-biphenyl]-2-yl)-6-methyl-1H-benzo[d]imidazole (1c').

${ }^{13}$ C NMR (175 MHz, DMSO-d $\left.)_{6}\right)$

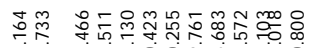

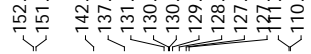
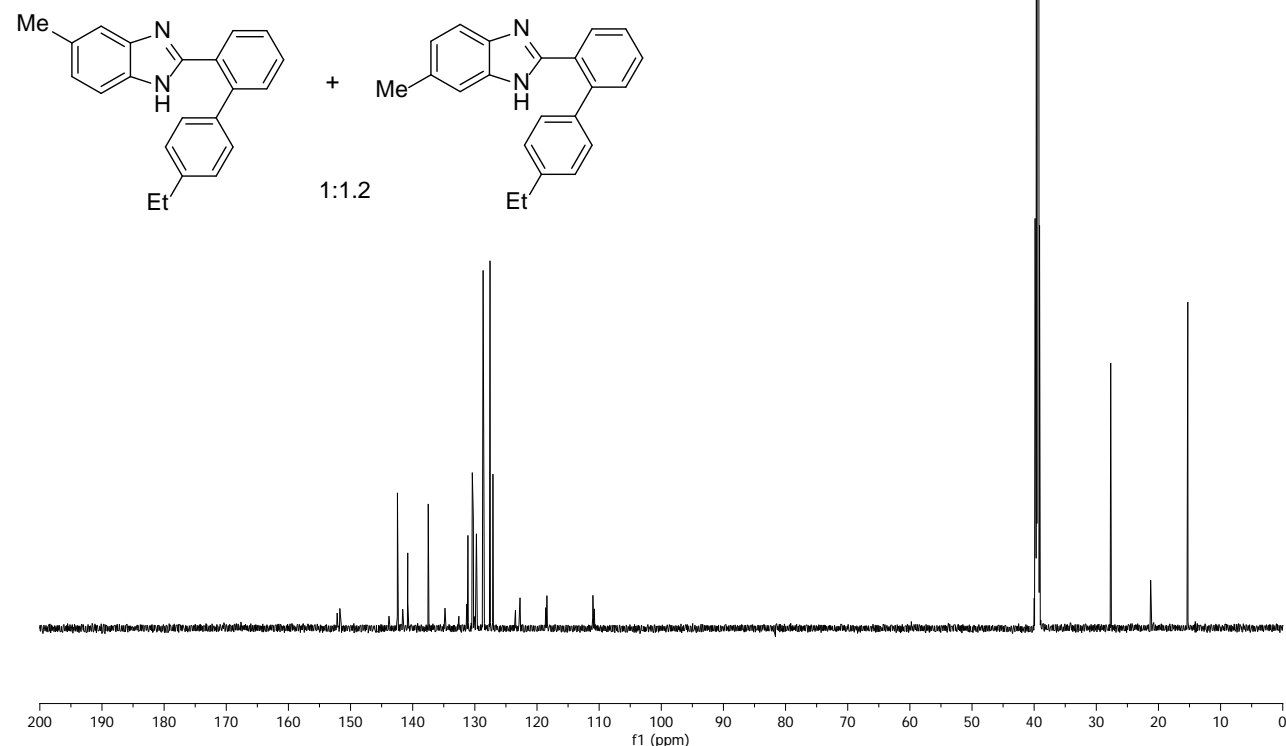

Figure S45. ${ }^{13} \mathrm{C}$ NMR spectrum of 2-(4'-Ethyl-[1,1'-biphenyl]-2-yl)-5-methyl-1Hbenzo[d]imidazole and 2-(4'-Ethyl-[1,1'-biphenyl]-2-yl)-6-methyl-1H-benzo[d]imidazole (1c'). 
${ }^{1} H_{\text {NMR (700 MHz, DMSO-d }}$ )

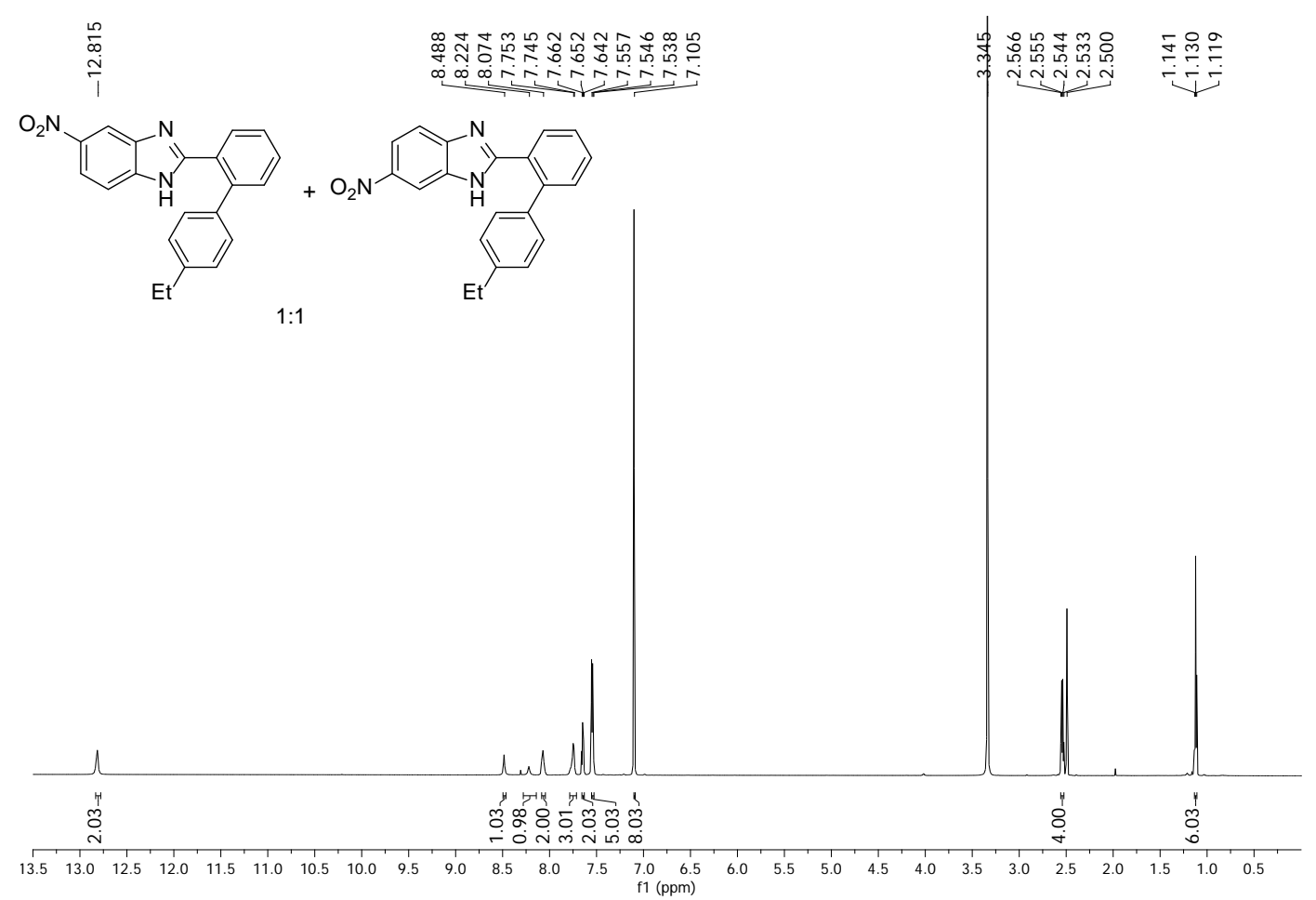

Figure S46. ${ }^{1} \mathrm{H}$ NMR spectrum of 2-(4'-Ethyl-[1,1'-biphenyl]-2-yl)-5-nitro-1Hbenzo[d]imidazole and 2-(4'-Ethyl-[1,1'-biphenyl]-2-yl)-6-nitro-1H-benzo[d]imidazole(1d').

${ }^{13}$ C NMR (175 MHz, DMSO-d ${ }_{6}$ )
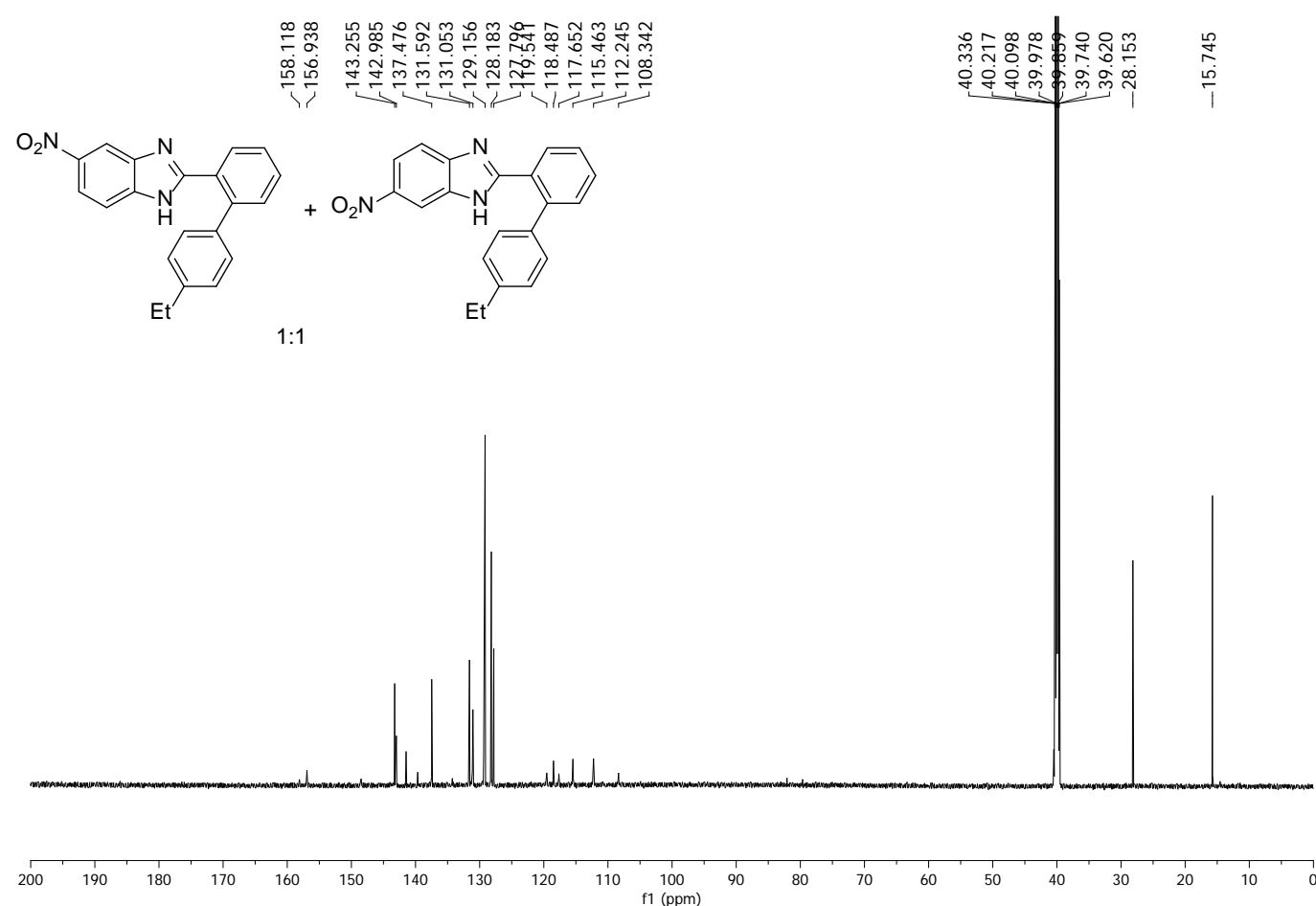

Figure S47. ${ }^{13} \mathrm{C}$ NMR spectrum of 2-(4'-Ethyl-[1,1'-biphenyl]-2-yl)-5-nitro-1Hbenzo[d]imidazole and 2-(4'-Ethyl-[1,1'-biphenyl]-2-yl)-6-nitro-1H-benzo[d]imidazole(1d'). 
${ }^{1}$ H NMR (700 MHz, DMSO-d 6 )

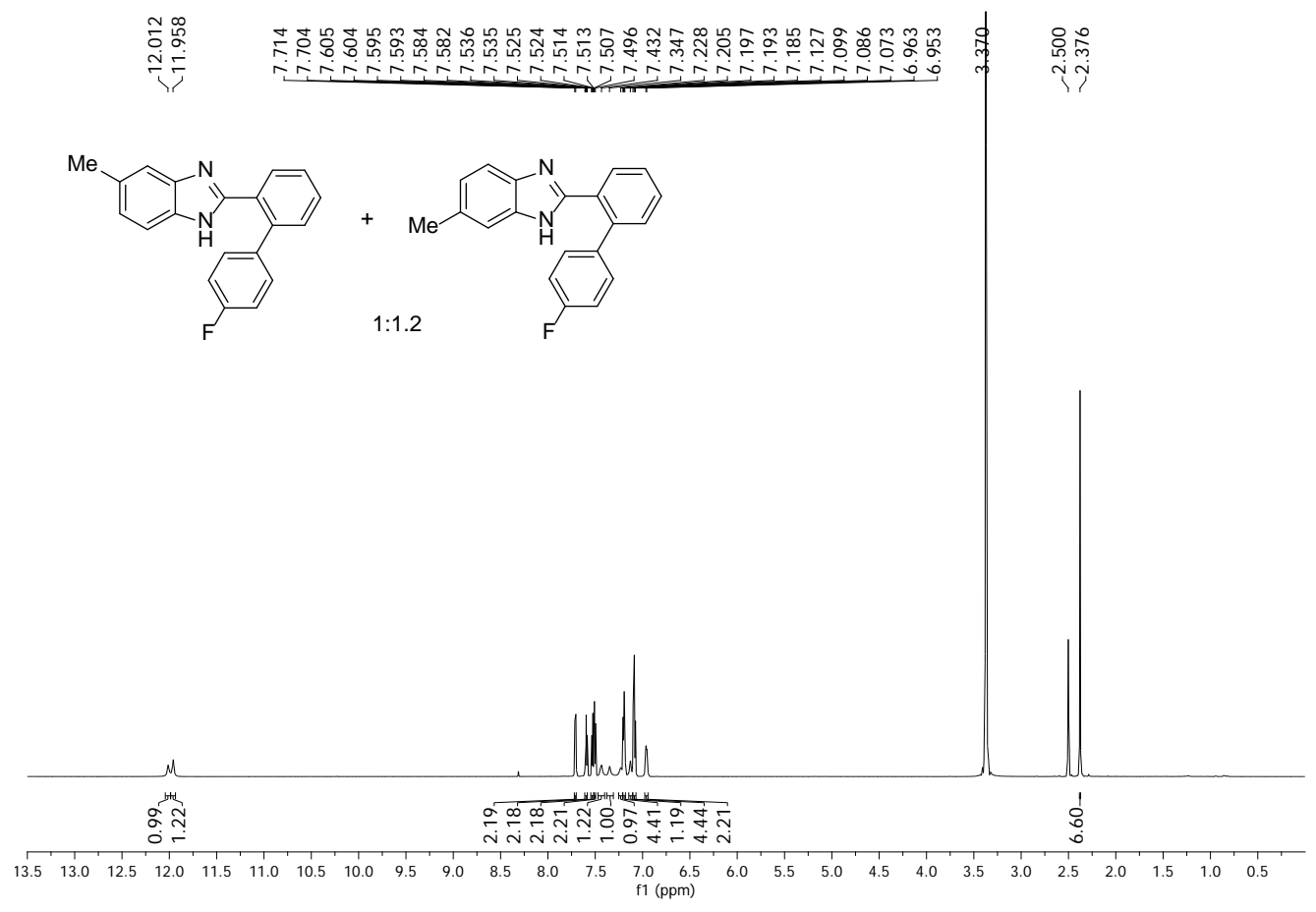

Figure S48. ${ }^{1} \mathrm{H}$ NMR spectrum of 2-(4'-Fluoro-[1,1'-biphenyl]-2-yl)-5-methyl-1Hbenzo[d]imidazole and 2-(4'-Fluoro-[1,1'-biphenyl]-2-yl)-6-methyl-1Hbenzo[d]imidazole(1e').

${ }^{13}$ C NMR (100 MHz, DMSO-d $)$

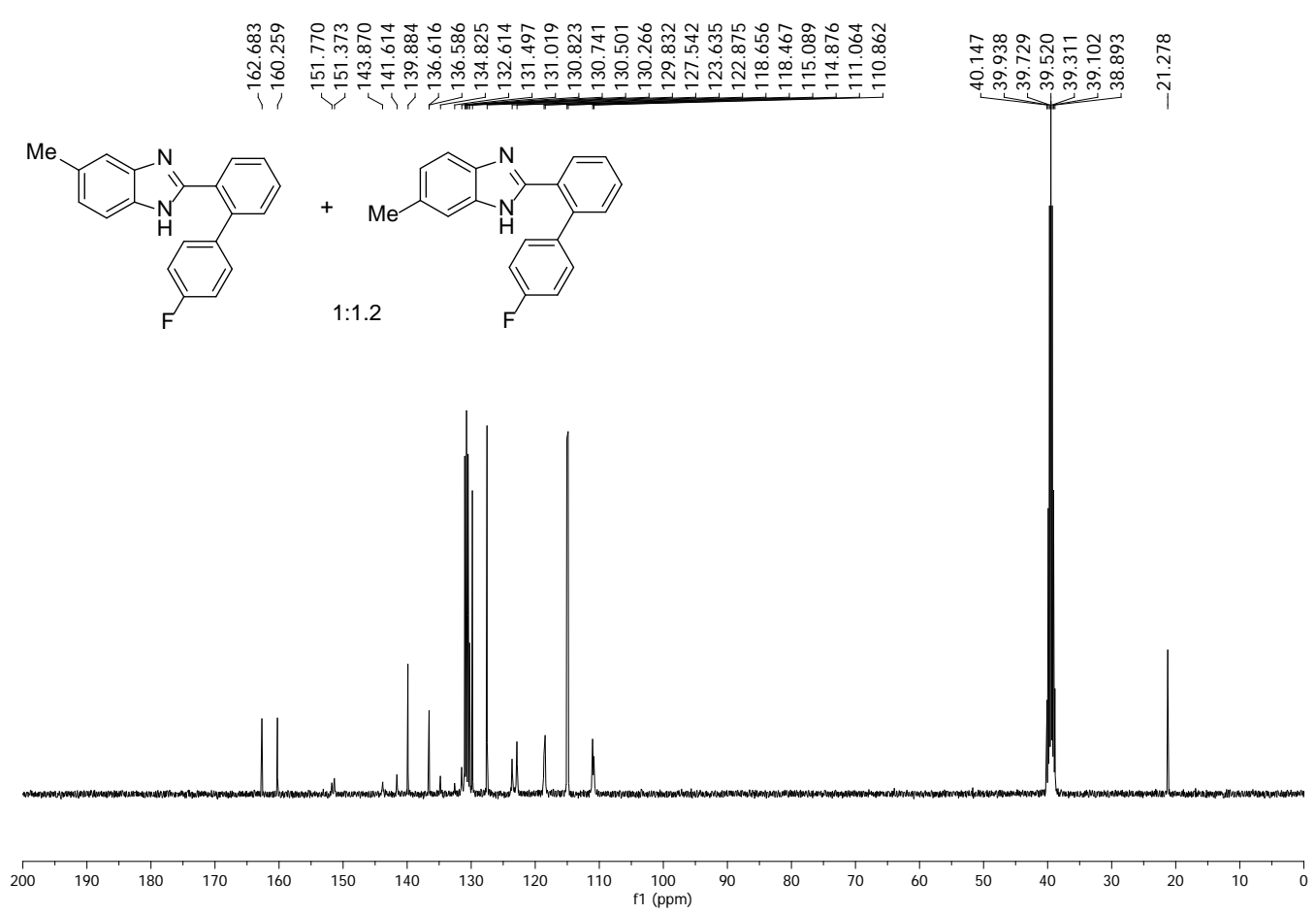

Figure S49. ${ }^{13} \mathrm{C}$ NMR spectrum of 2-(4'-Fluoro-[1,1'-biphenyl]-2-yl)-5-methyl-1Hbenzo[d]imidazole and 2-(4'-Fluoro-[1,1'-biphenyl]-2-yl)-6-methyl-1Hbenzo[d]imidazole(1e'). 
${ }^{1}$ H NMR (400 MHz, DMSO-d $\left.{ }_{6}\right)$

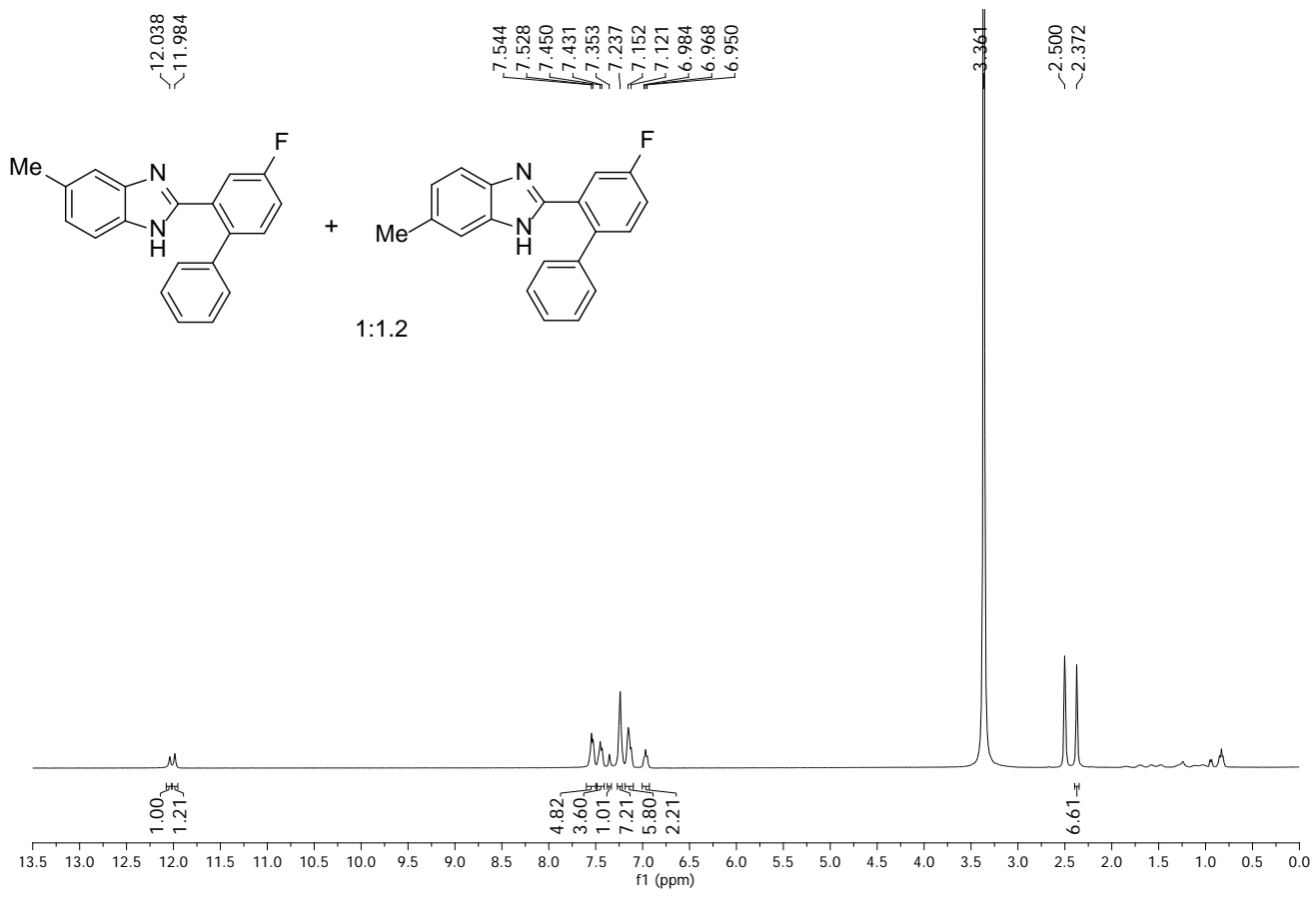

Figure S50. ${ }^{1} \mathrm{H}$ NMR spectrum of 2-(4-Fluoro-[1,1'-biphenyl]-2-yl)-5-methyl-1Hbenzo[d]imidazole and 2-(4-Fluoro-[1,1'-biphenyl]-2-yl)-6-methyl-1H-benzo[d]imidazole (1f').

${ }^{13}$ C NMR (100 MHz, DMSO-d $\left.{ }_{6}\right)$

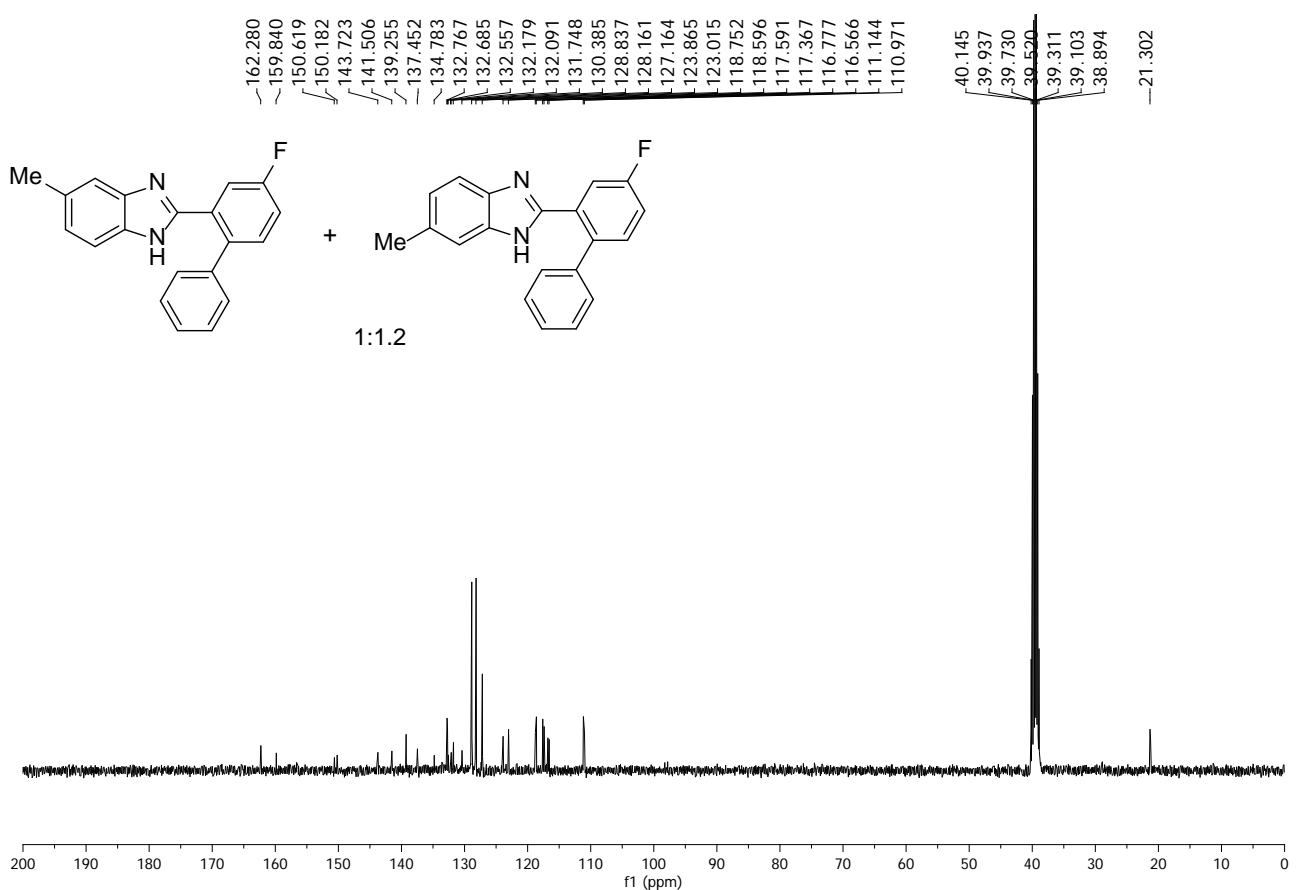

Figure S51. ${ }^{13} \mathrm{C}$ NMR spectrum of 2-(4-Fluoro-[1,1'-biphenyl]-2-yl)-5-methyl-1Hbenzo[d]imidazole and 2-(4-Fluoro-[1,1'-biphenyl]-2-yl)-6-methyl-1H-benzo[d]imidazole (1f'). 
${ }^{1}$ H NMR (700 MHz, DMSO-d ${ }_{6}$ )

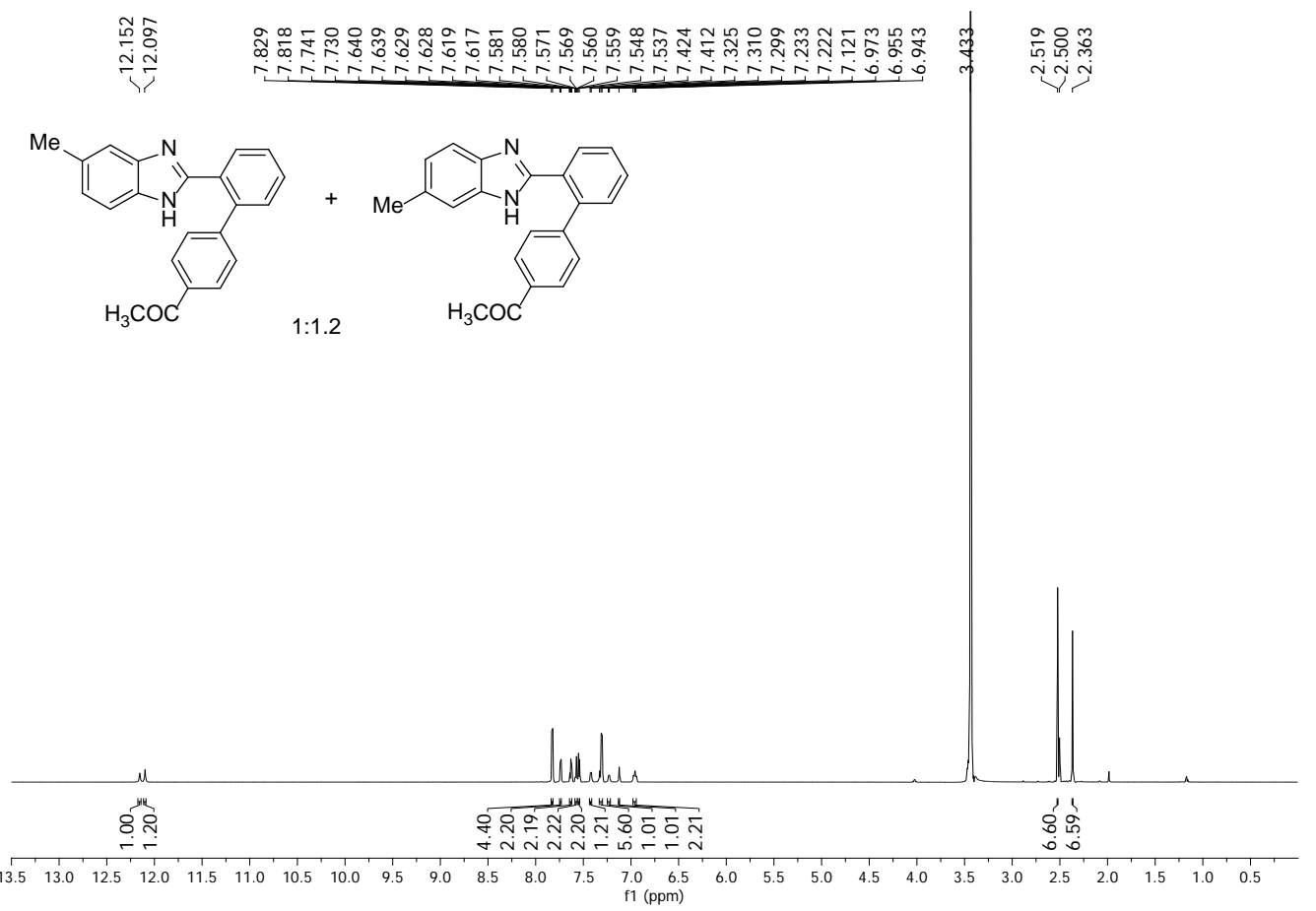

Figure S52. ${ }^{1} \mathrm{H}$ NMR spectrum of 1-(2'-(5-Methyl-1H-benzo[d]imidazol-2-yl)-[1,1'biphenyl]-4-yl)ethan-1-one and 1-(2'-(6-Methyl-1H-benzo[d]imidazol-2-yl)-[1,1'-biphenyl]4-yl)ethan-1-one (19').

${ }^{13}$ C NMR (175 MHz, DMSO-d 6 )

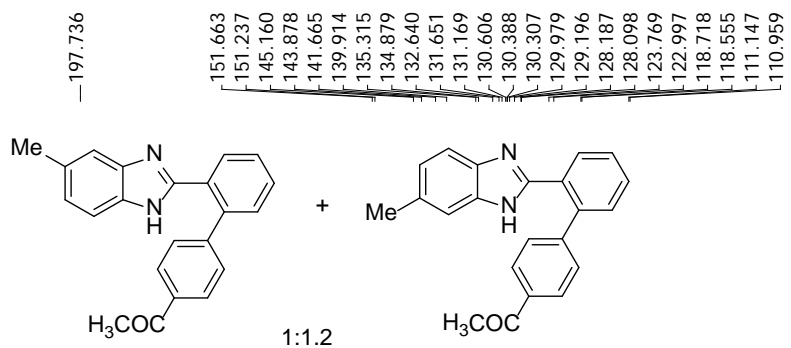

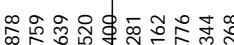

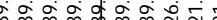
m mm ñ

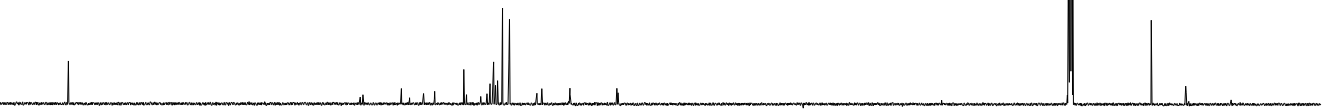

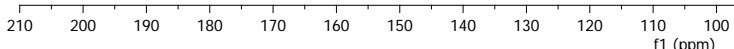

Figure S53. ${ }^{13} \mathrm{C}$ NMR spectrum of 1-(2'-(5-Methyl-1H-benzo[d]imidazol-2-yl)-[1,1'biphenyl]-4-yl)ethan-1-one and 1-(2'-(6-Methyl-1H-benzo[d]imidazol-2-yl)-[1,1'-biphenyl]4-yl)ethan-1-one (19') 
${ }^{1} \mathrm{H}$ NMR (700 MHz, $\mathrm{CDCl}_{3}$ )

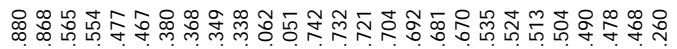

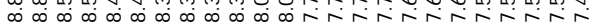<smiles>c1ccc2c(c1)nc1c3ccccc3c3ccccc3n21</smiles>

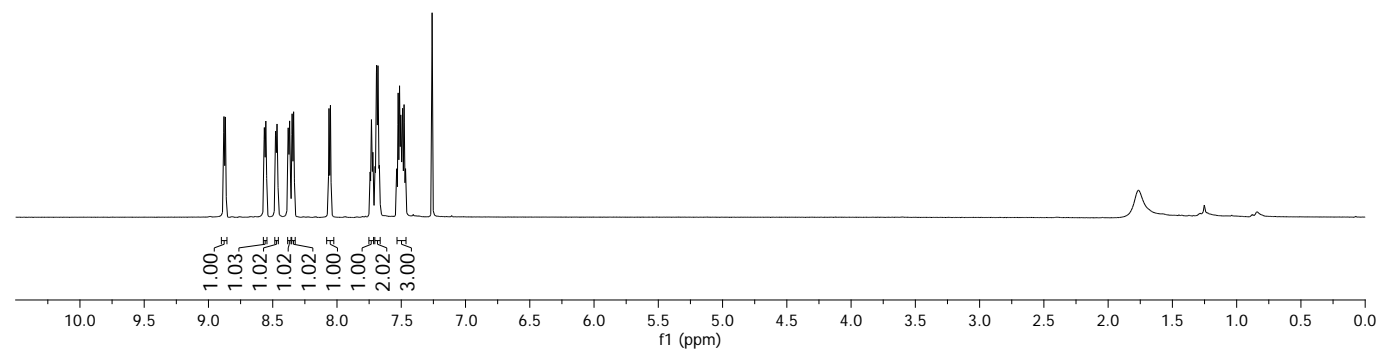

Figure S54. ${ }^{1} \mathrm{H}$ NMR spectrum of Benzo[4,5]imidazo[1,2-f]phenanthridine (2a).

${ }^{1} \mathrm{H}$ NMR (700 MHz, $\mathrm{CDCl}_{3}$ )

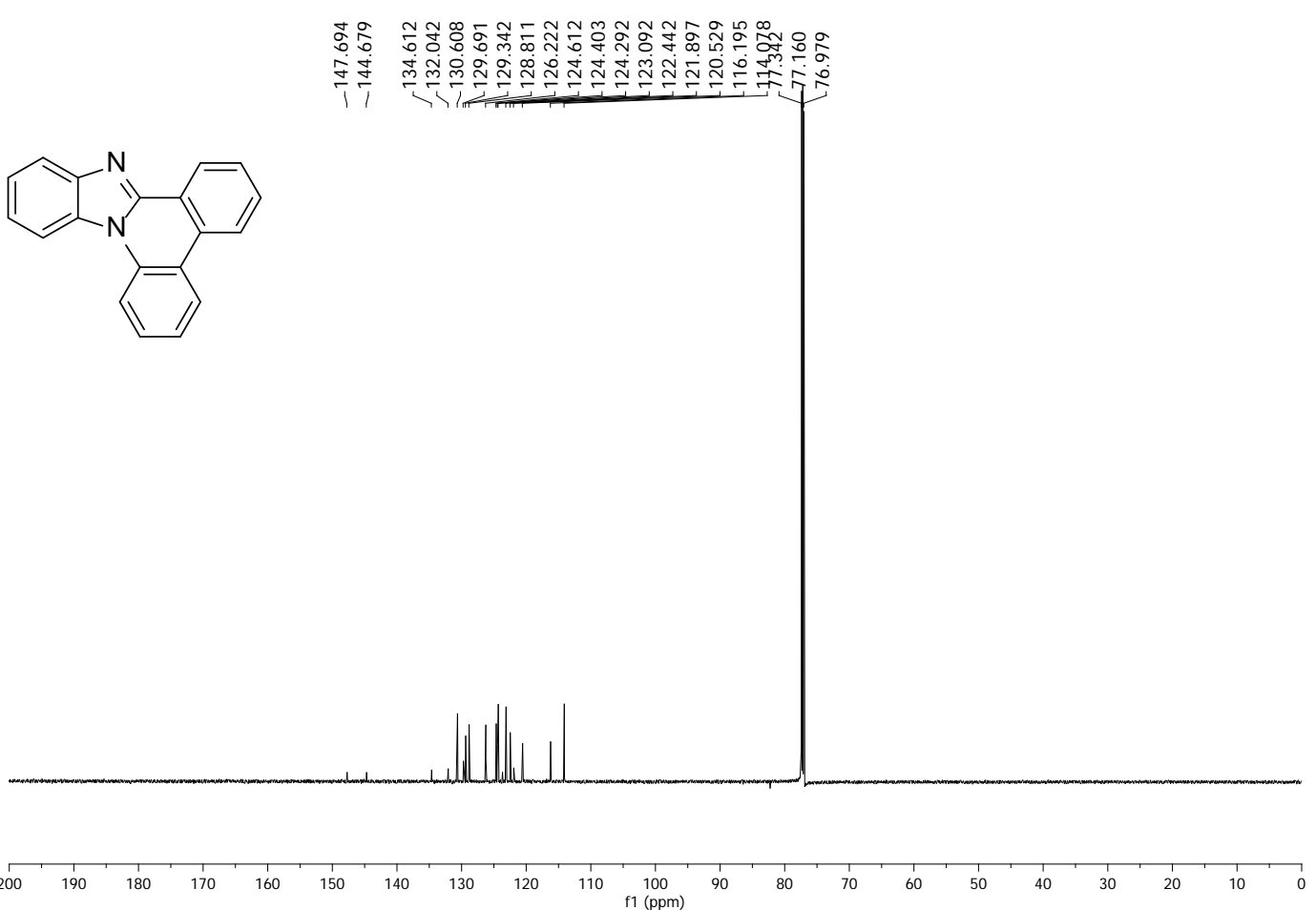

Figure S55. ${ }^{13} \mathrm{C}$ NMR spectrum of Benzo[4,5]imidazo[1,2-f]phenanthridine (2a). 
${ }^{1} \mathrm{H}$ NMR (700 MHz, $\left.\mathrm{CDCl}_{3}\right)$

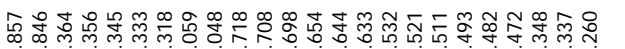

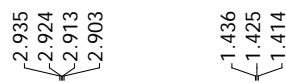

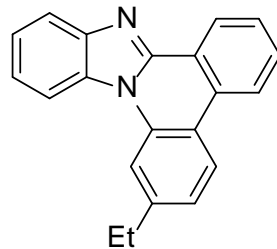

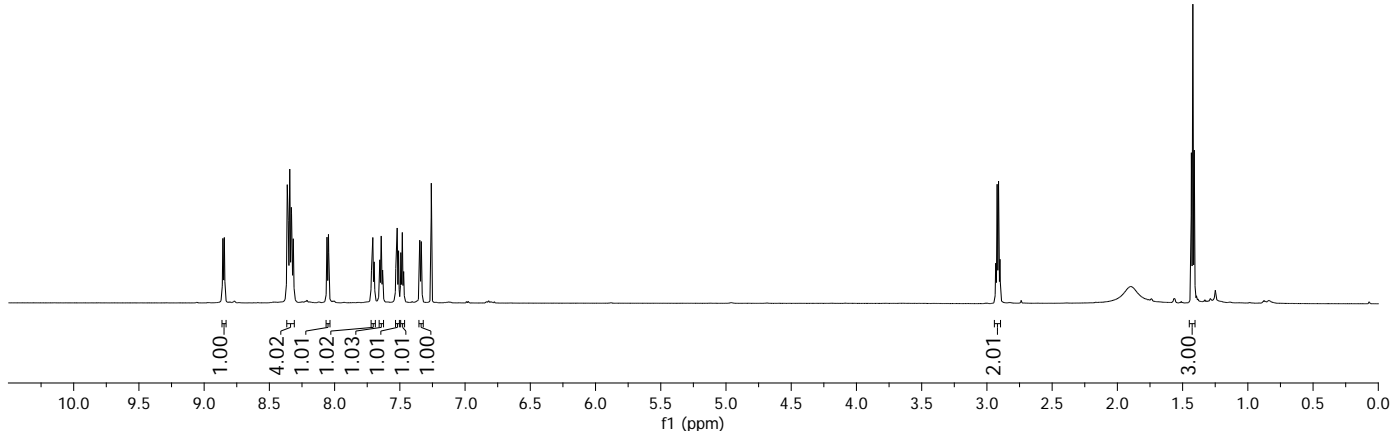

Figure S56. ${ }^{1} \mathrm{H}$ NMR spectrum of 2-Ethylbenzo[4,5]imidazo[1,2-f]phenanthridine (2b). ${ }^{13} \mathrm{C}$ NMR (175 MHz, $\mathrm{CDCl}_{3}$ )

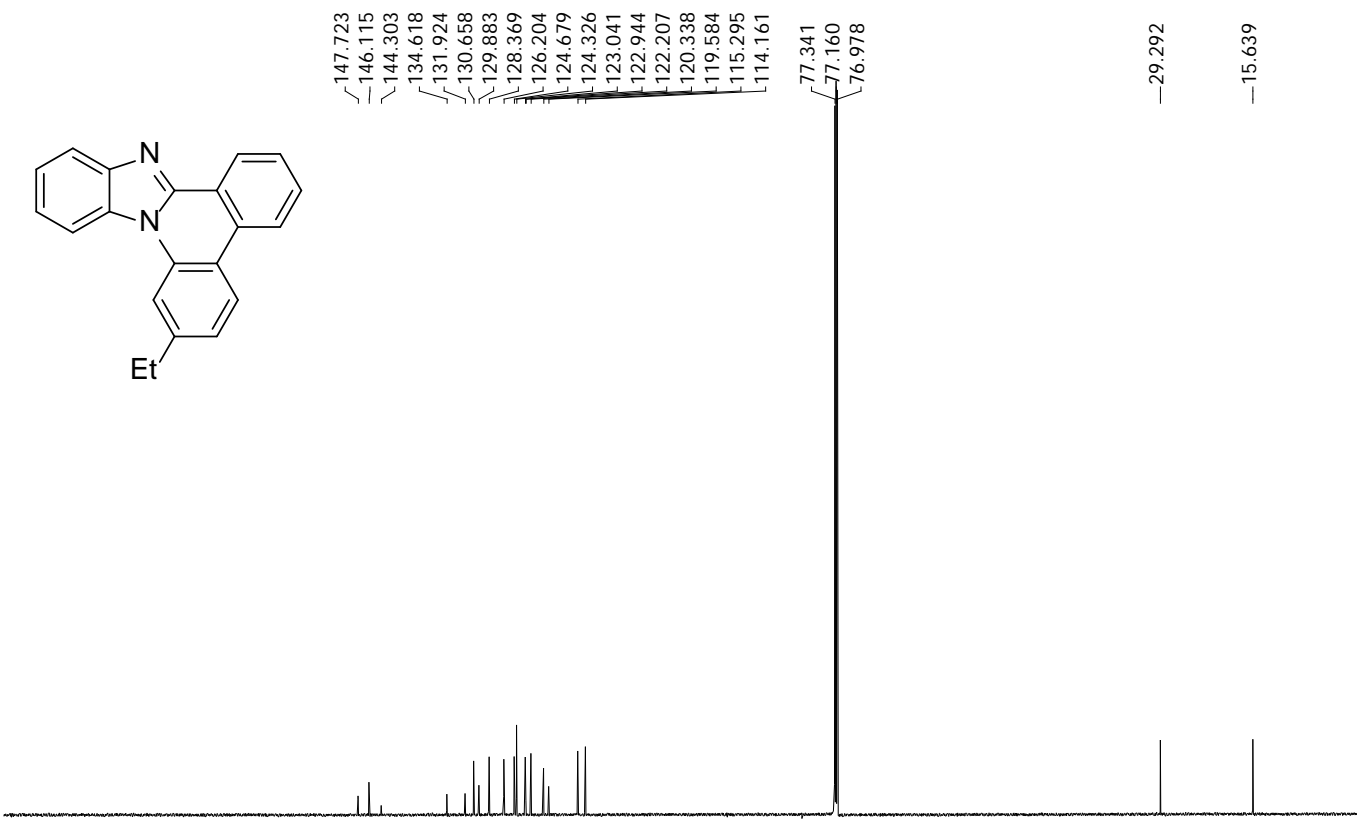

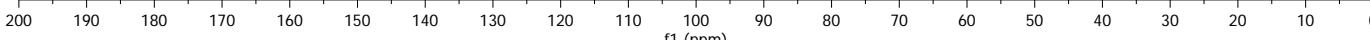

Figure S57. ${ }^{13} \mathrm{C}$ NMR spectrum of 2-Ethylbenzo[4,5]imidazo[1,2-f]phenanthridine (2b). 


\section{${ }^{1} \mathrm{H}$ NMR (700 MHz, $\left.\mathrm{CDCl}_{3}\right)$}

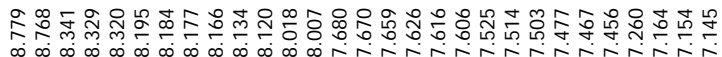

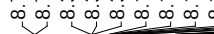<smiles>Fc1ccc2c(c1)c1ccccc1n1c3ccccc3nc21</smiles>

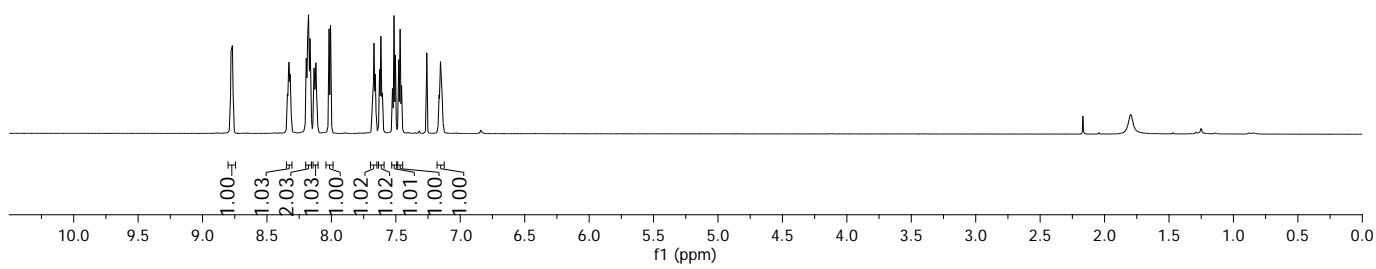

Figure S58. ${ }^{1} \mathrm{H}$ NMR spectrum of 2-Fluorobenzo[4,5]imidazo[1,2-f]phenanthridine (2c). ${ }^{13} \mathrm{C}$ NMR (175 MHz, $\left.\mathrm{CDCl}_{3}\right)$

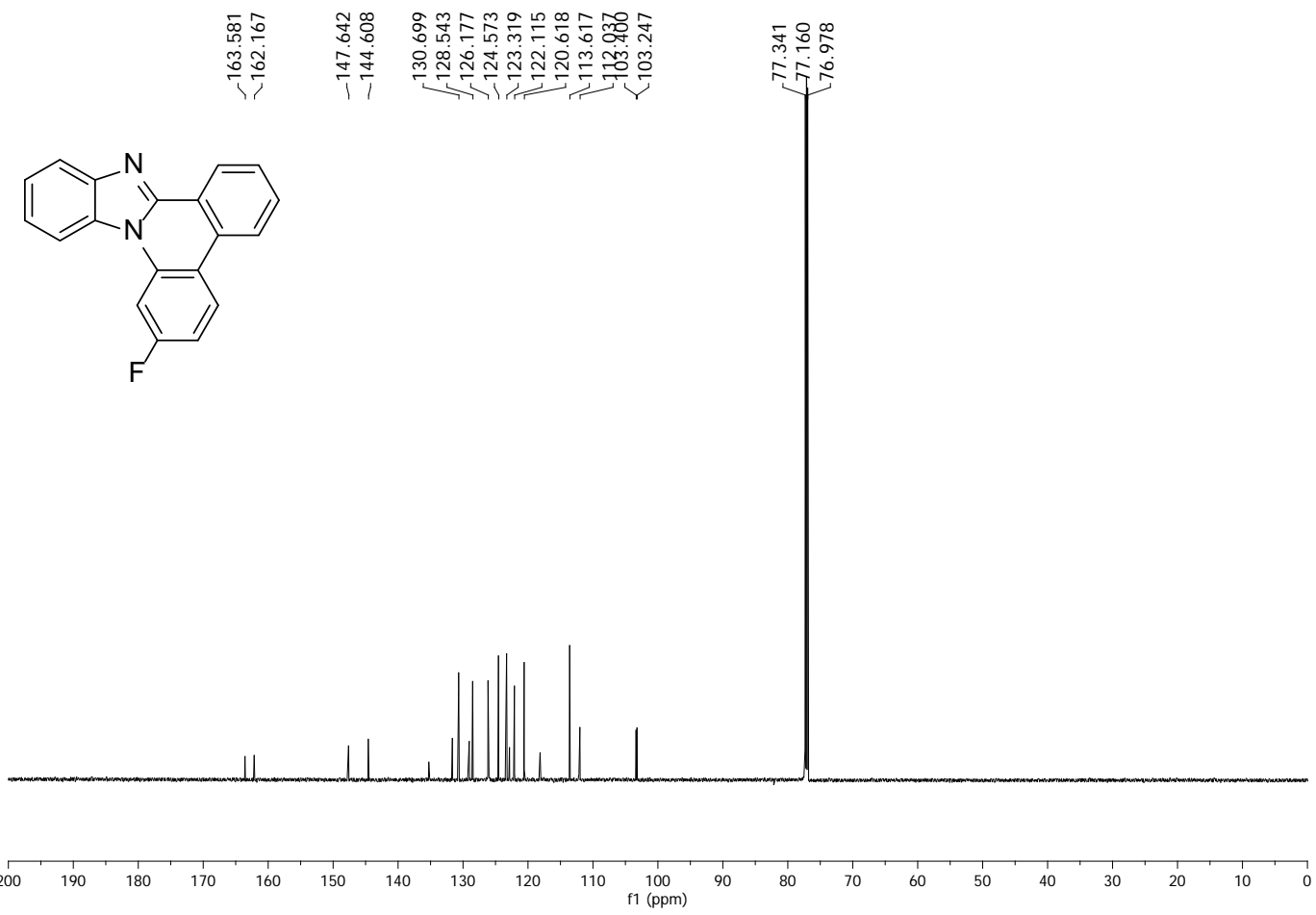

Figure S59. ${ }^{13} \mathrm{C}$ NMR spectrum of 2-Fluorobenzo[4,5]imidazo[1,2-f]phenanthridine (2c). 
${ }^{1} \mathrm{H}$ NMR (700 MHz, $\mathrm{CDCl}_{3}$ )

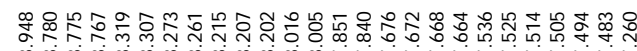

舟<smiles>CC(=O)c1ccc2c3ccccc3c3nc4ccccc4n3c2c1</smiles>

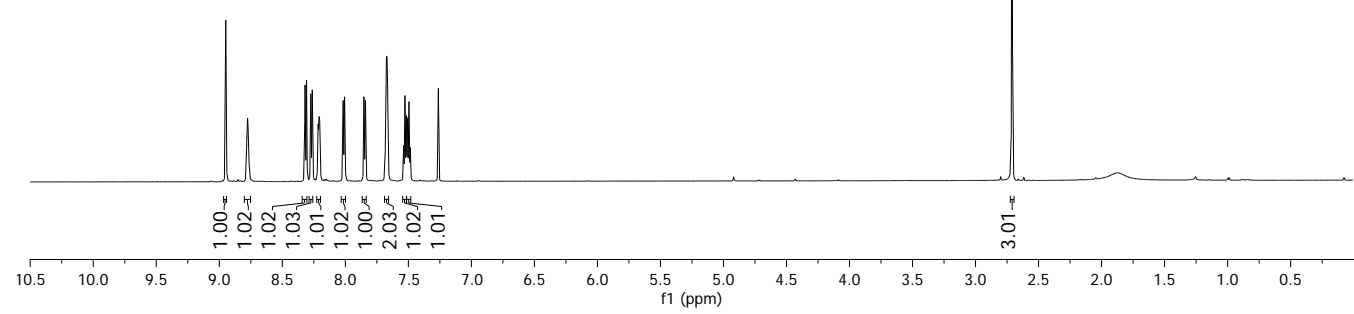

Figure S60. ${ }^{1} \mathrm{H}$ NMR spectrum of 1-(Benzo[4,5]imidazo[1,2-f]phenanthridin-2-yl)ethan-1one (2d).

${ }^{13} \mathrm{C}$ NMR (100 MHz, $\left.\mathrm{CDCl}_{3}\right)$

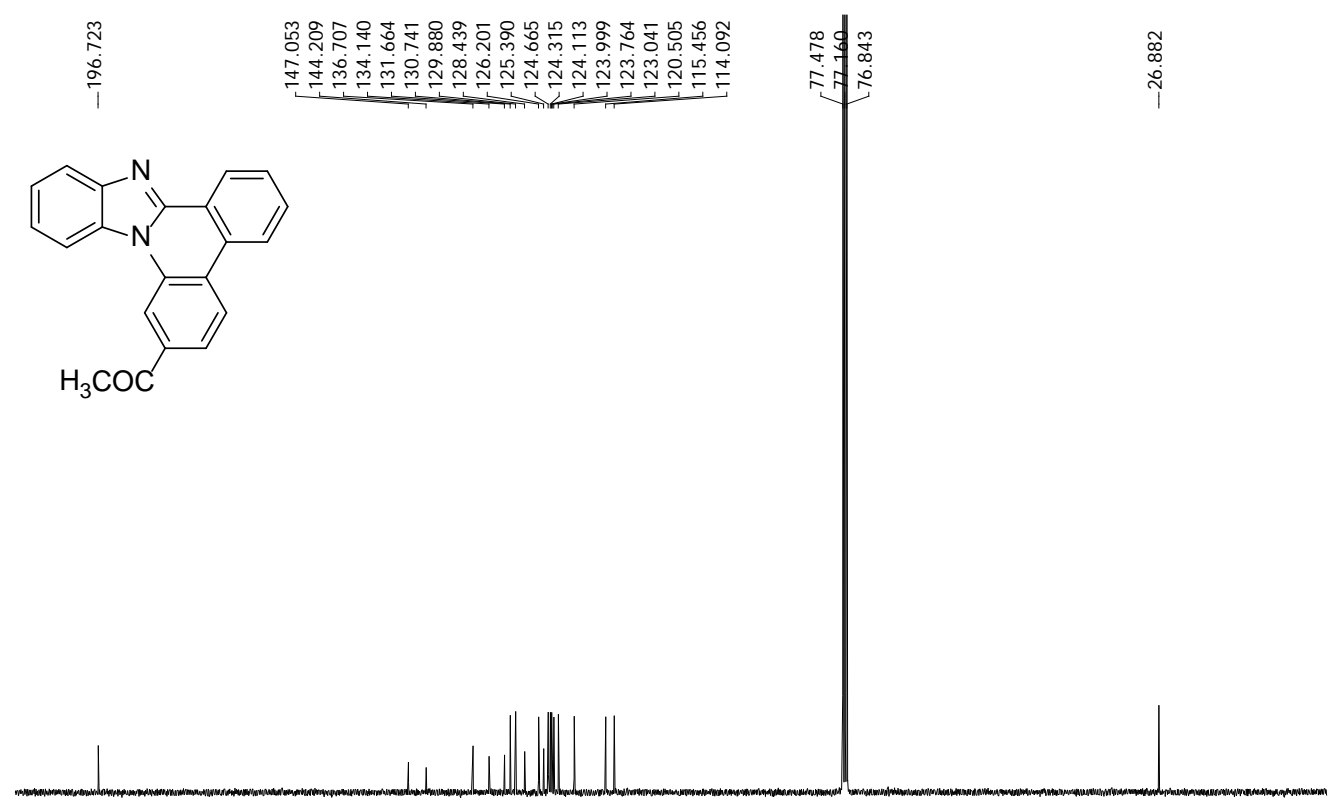

$\begin{array}{llllllllllllllllllllllll}\mid & 190 & 190 & 180 & 170 & 160 & 150 & 140 & 130 & 120 & \underset{f 1}{1}(\mathrm{ppm}) & 100 & 90 & 80 & 70 & 60 & 50 & 40 & 30 & 20 & 10 & 0\end{array}$

Figure S61. ${ }^{13} \mathrm{C}$ NMR spectrum of 1-(Benzo[4,5]imidazo[1,2-f]phenanthridin-2-yl)ethan-1one (2d). 
${ }^{1} \mathrm{H}$ NMR (700 MHz, $\left.\mathrm{CDCl}_{3}\right)$

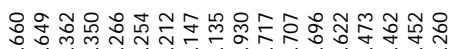

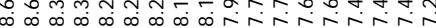<smiles></smiles>

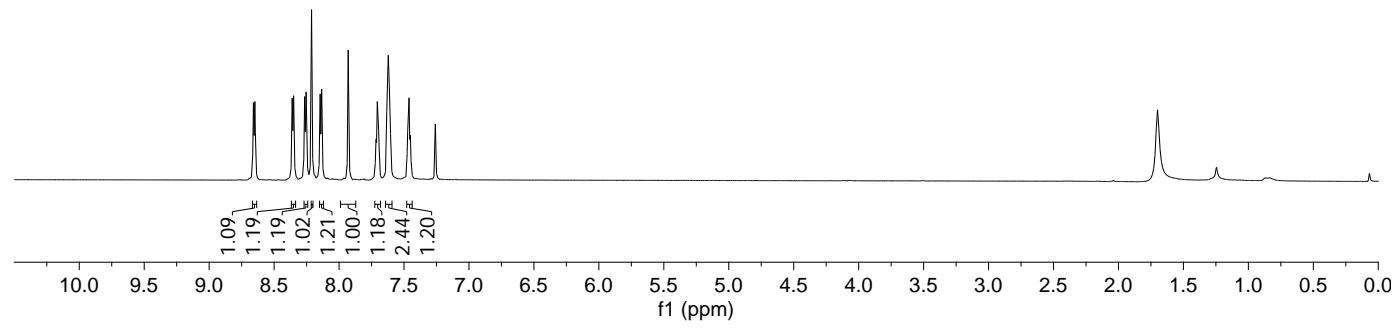

Figure S62. ${ }^{1} \mathrm{H}$ NMR spectrum of 11,12-Dichlorobenzo[4,5]imidazo[1,2-f]phenanthridine (2e).

${ }^{13} \mathrm{C}$ NMR (175 MHz, $\mathrm{CDCl}_{3}$ )

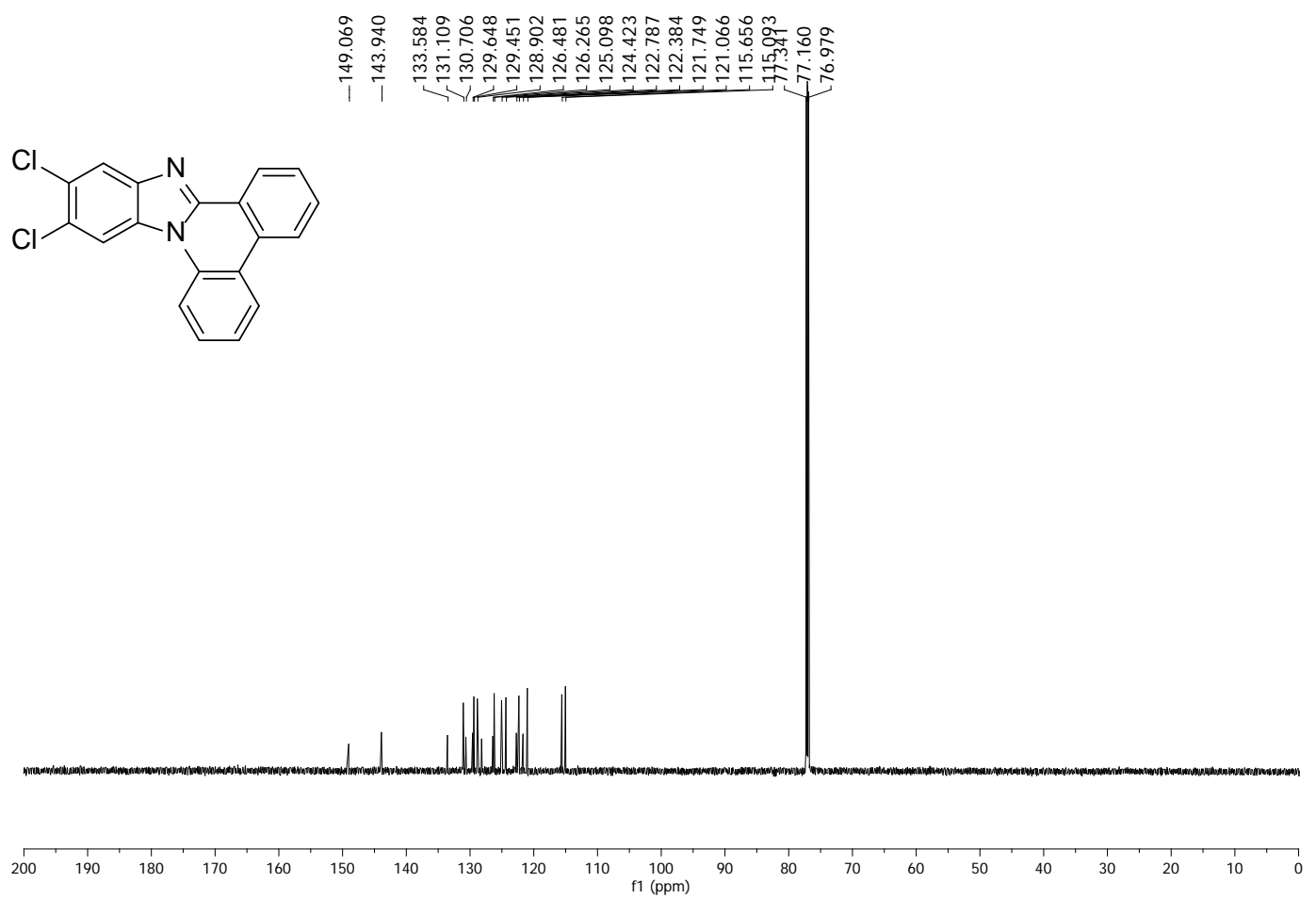

Figure S63. ${ }^{13} \mathrm{C}$ NMR spectrum of 11,12-Dichlorobenzo[4,5]imidazo[1,2-f]phenanthridine (2e) 
${ }^{1} \mathrm{H}$ NMR (700 MHz, $\left.\mathrm{CDCl}_{3}\right)$

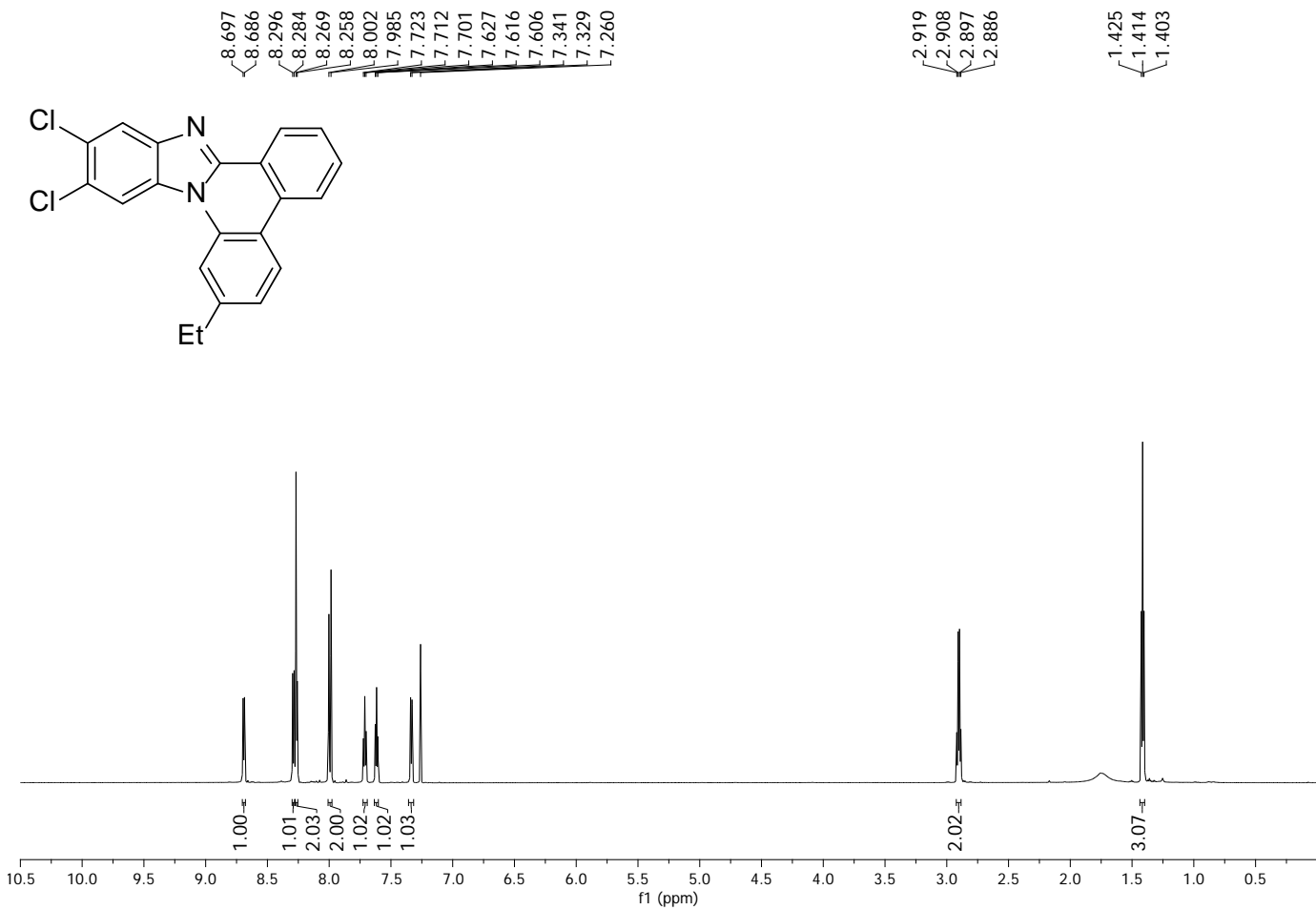

Figure S64. ${ }^{1} \mathrm{H}$ NMR spectrum of 11,12-Dichloro-2-ethylbenzo[4,5]imidazo[1,2f]phenanthridine (2f).

${ }^{1} \mathrm{H}$ NMR (700 MHz, $\left.\mathrm{CDCl}_{3}\right)$

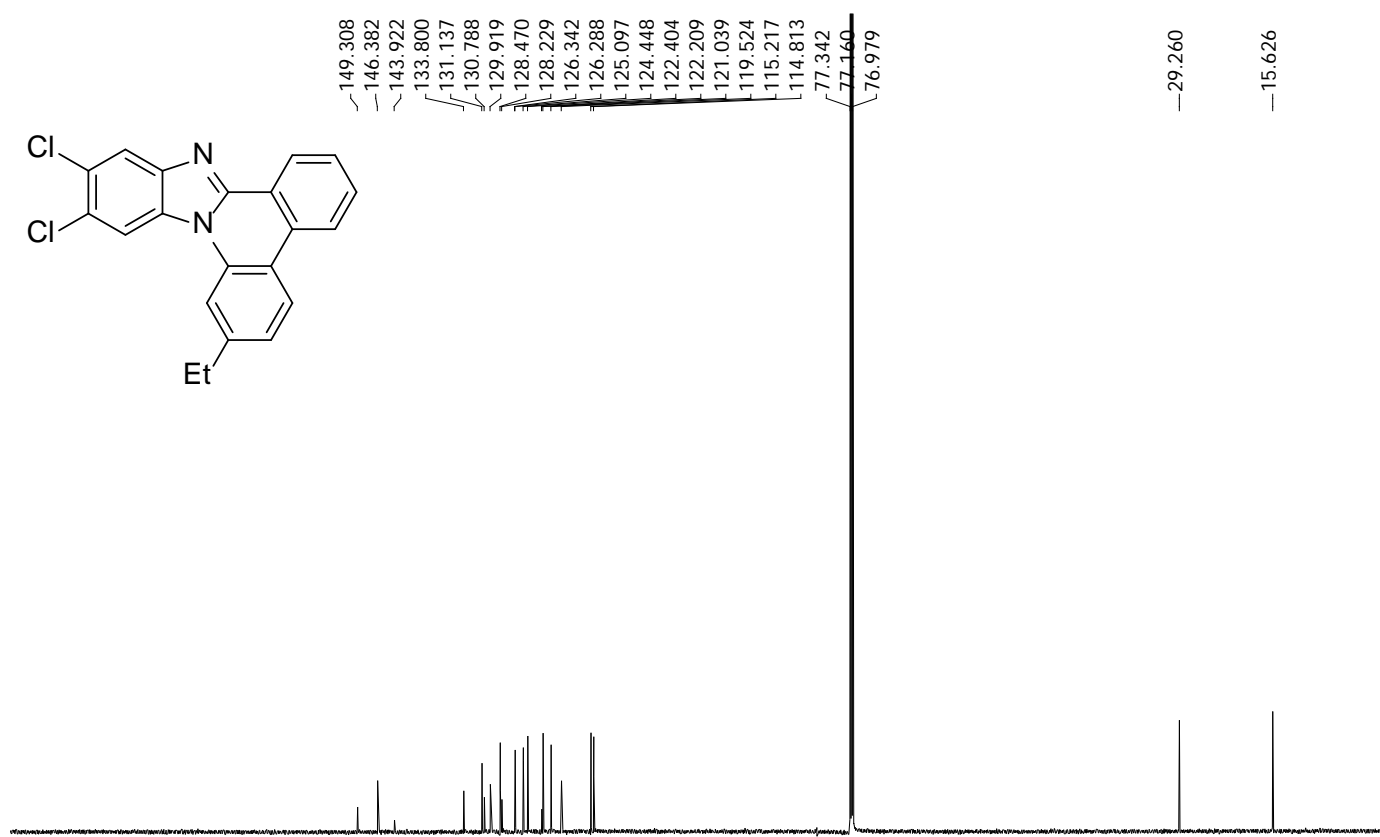

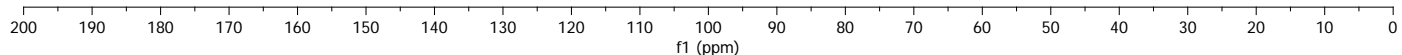

Figure S65. ${ }^{13} \mathrm{C}$ NMR spectrum of 11,12-Dichloro-2-ethylbenzo[4,5]imidazo[1,2f]phenanthridine (2f). 


\section{${ }^{1} \mathrm{H}$ NMR (700 MHz, $\left.\mathrm{CDCl}_{3}\right)$}

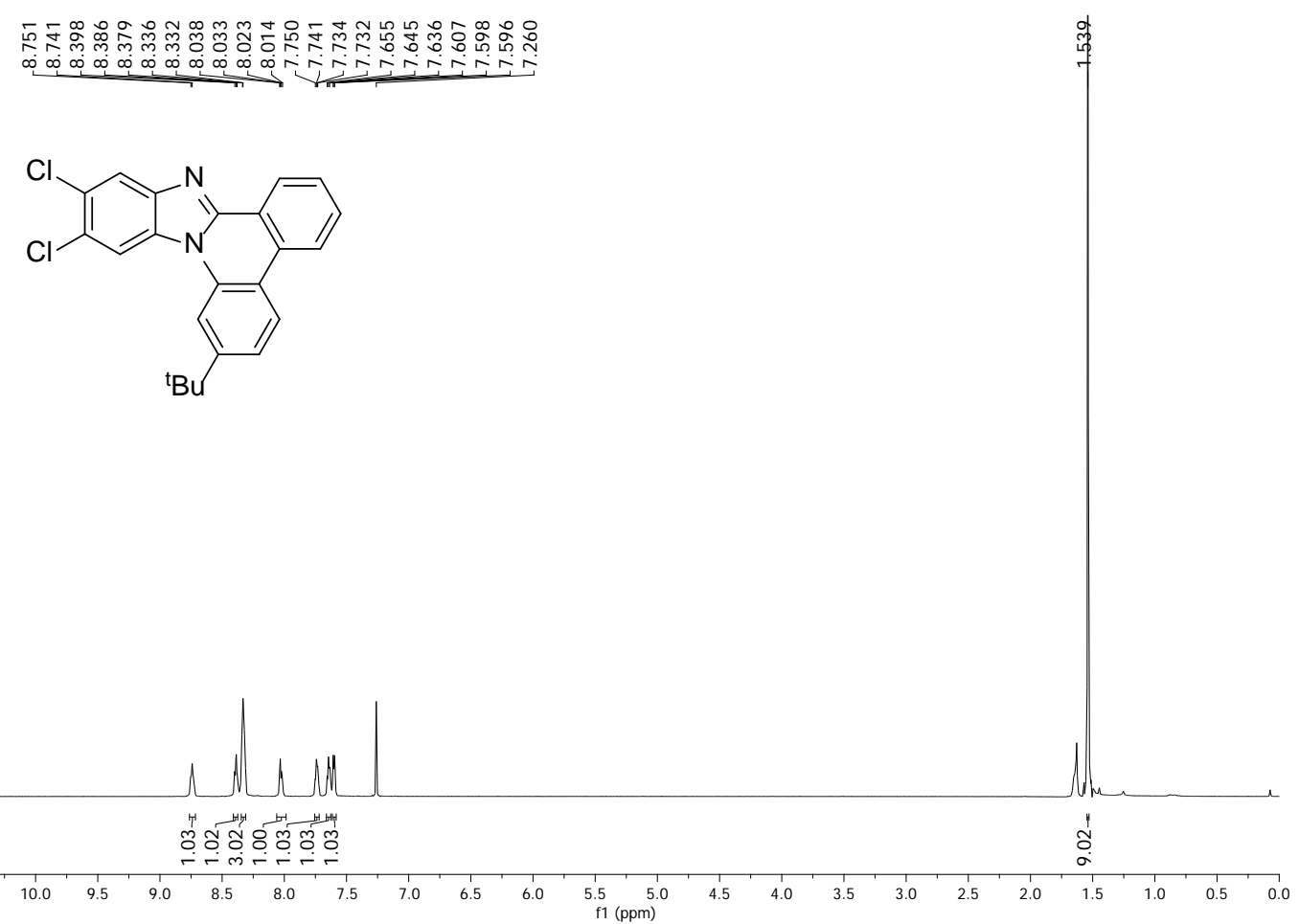

Figure S66. ${ }^{1} \mathrm{H}$ NMR spectrum of 2-(Tert-butyl)-11,12-dichlorobenzo[4,5]imidazo[1,2f]phenanthridine (2g).

${ }^{13} \mathrm{C}$ NMR (175 MHz, $\left.\mathrm{CDCl}_{3}\right)$

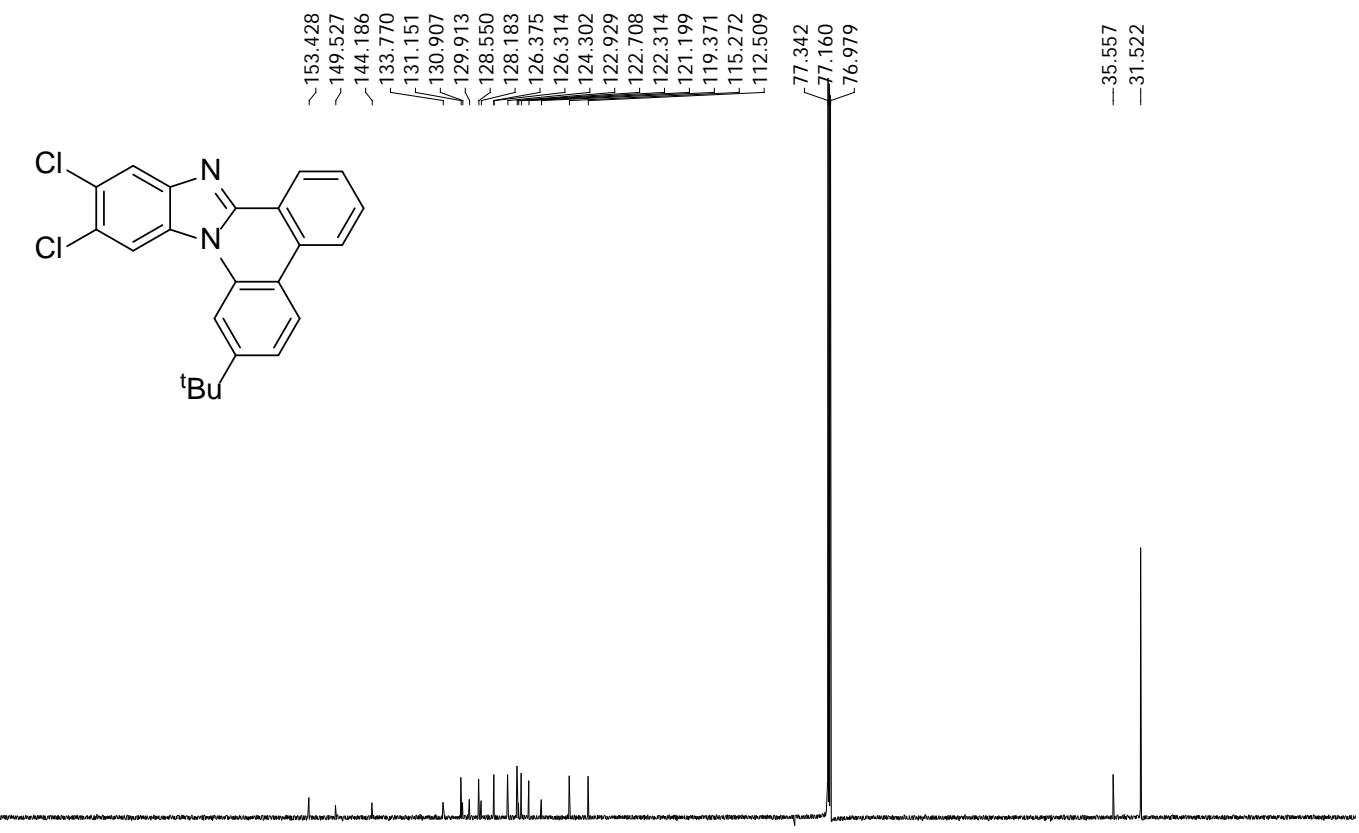

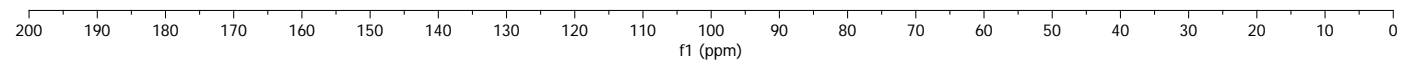

Figure S67. ${ }^{13} \mathrm{C}$ NMR spectrum of 2-(Tert-butyl)-11,12-dichlorobenzo[4,5]imidazo[1,2f]phenanthridine (2g). 
${ }^{1} \mathrm{H}$ NMR (400 MHz, $\mathrm{CDCl}_{3}+$ TFA-D)

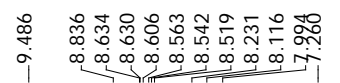

(l)

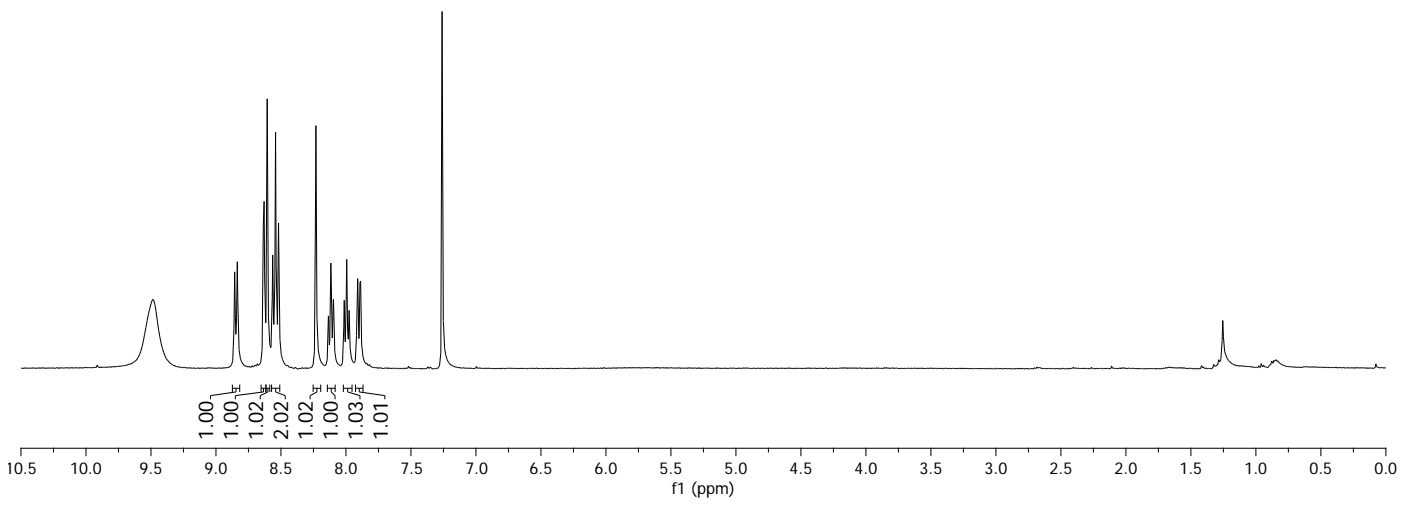

Figure S68. ${ }^{1} \mathrm{H}$ NMR spectrum of 3,11,12-Trichlorobenzo[4,5]imidazo[1,2-f]phenanthridine (2h).
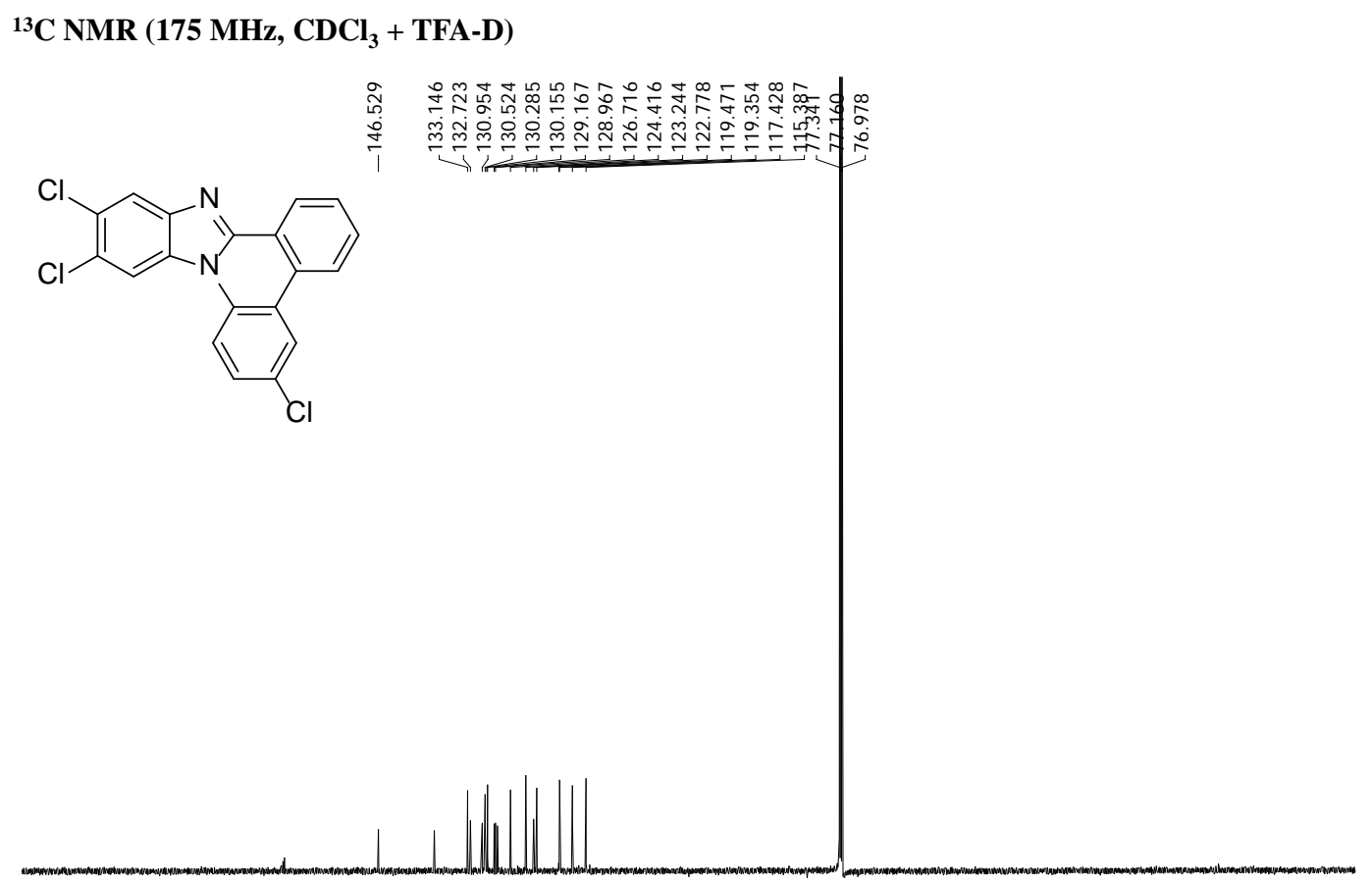

$\begin{array}{llllllllllllllllllllll} & 200 & 190 & 180 & 170 & 160 & 150 & 140 & 130 & 120 & 110 & 100 & 90 & 80 & 70 & 60 & 50 & 40 & 30 & 20 & 10 & 0\end{array}$

Figure S69. ${ }^{13} \mathrm{C}$ NMR spectrum of 3,11,12-Trichlorobenzo[4,5]imidazo[1,2-f]phenanthridine (2h). 
${ }^{1} \mathrm{H}$ NMR (700 MHz, $\mathrm{CDCl}_{3}+$ TFA-D)
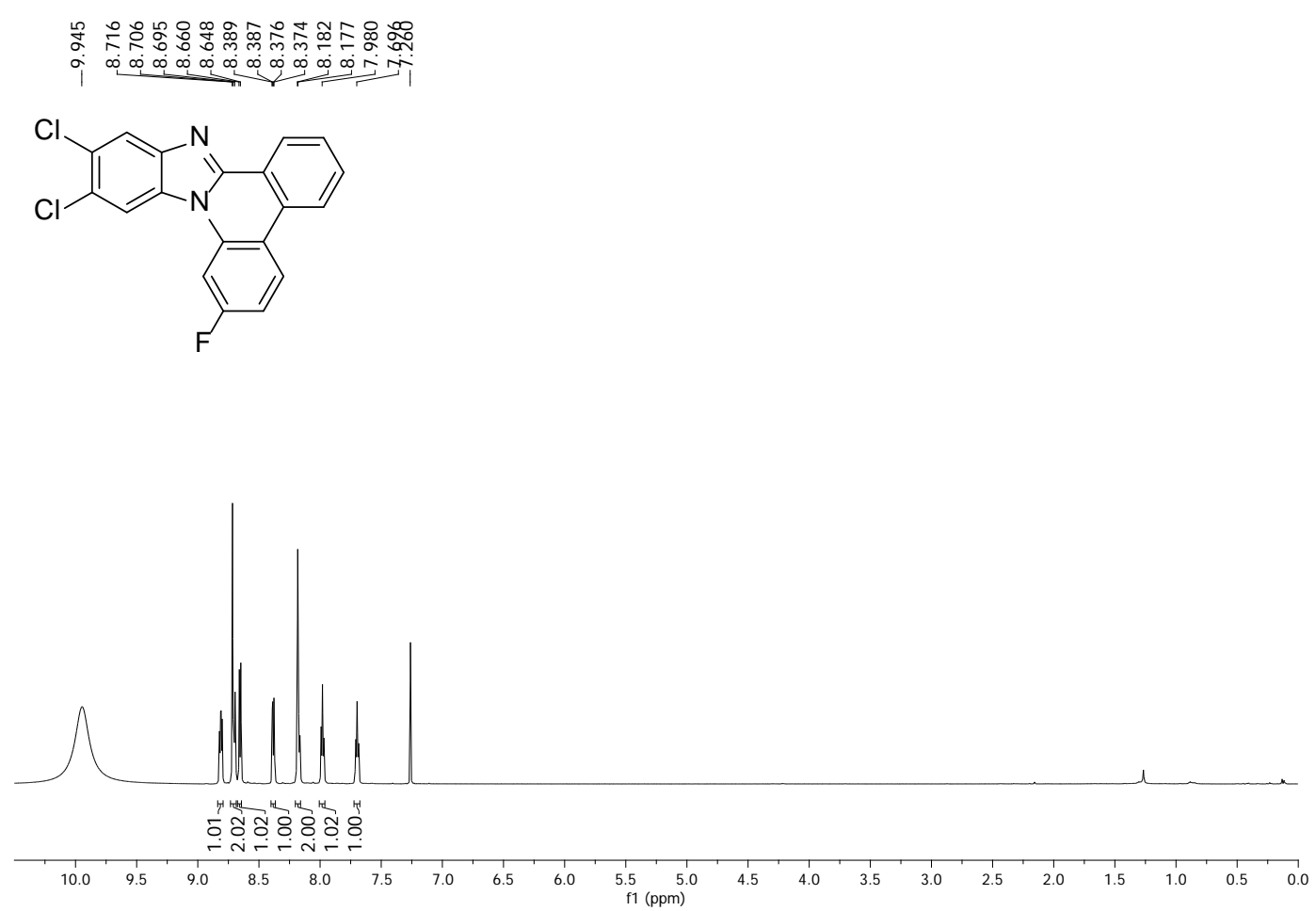

Figure S70. ${ }^{1} \mathrm{H}$ NMR spectrum of 11,12-Dichloro-2-fluorobenzo[4,5]imidazo[1,2f]phenanthridine (2i).

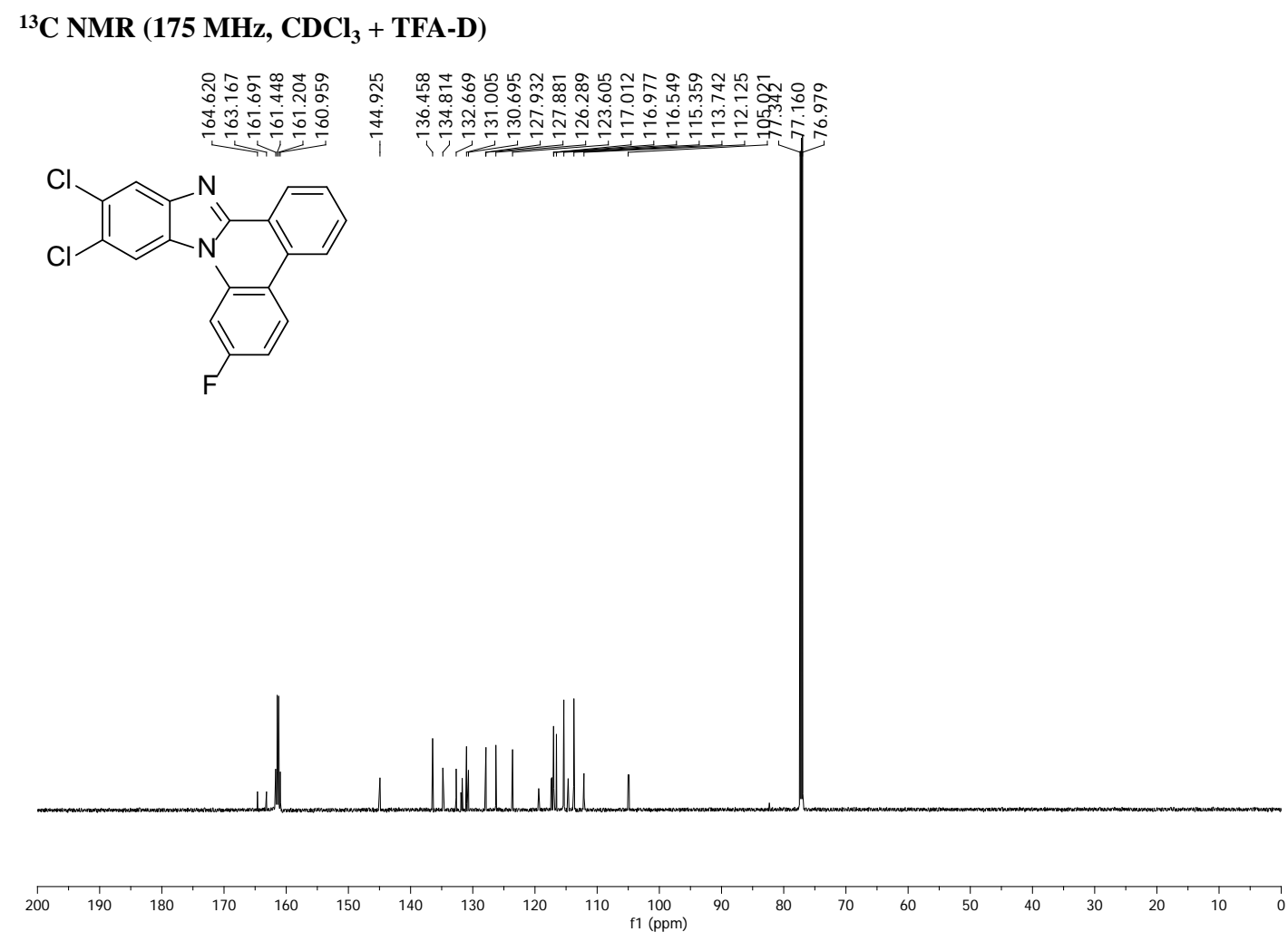

Figure S71. ${ }^{13} \mathrm{C}$ NMR spectrum of 11,12-Dichloro-2-fluorobenzo[4,5]imidazo[1,2f]phenanthridine (2i). 


\section{${ }^{1} \mathrm{H}$ NMR (400 MHz, $\left.\mathrm{CDCl}_{3}\right)$}
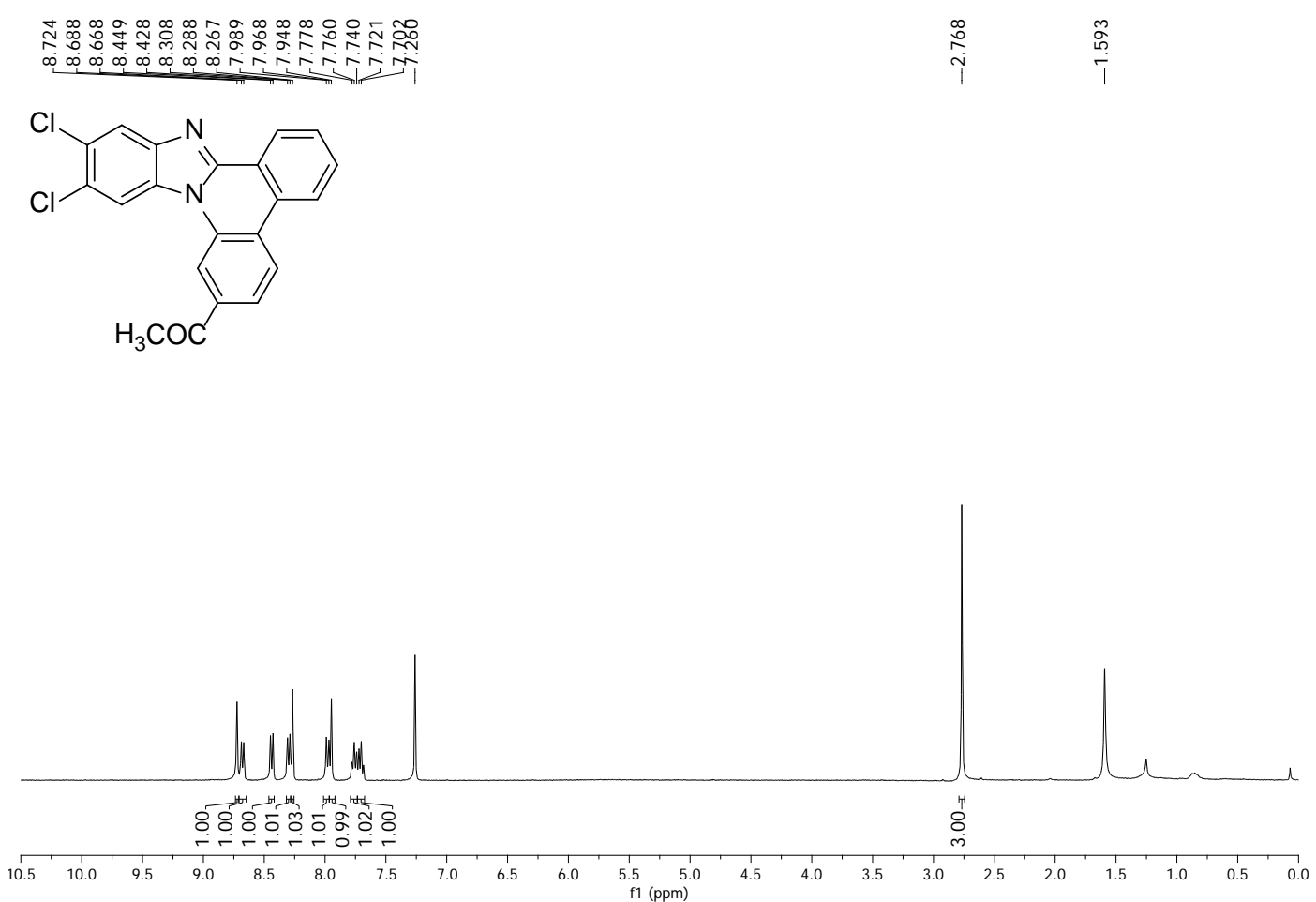

Figure S72. ${ }^{1} \mathrm{H}$ NMR spectrum of 1-(11,12-Dichlorobenzo[4,5]imidazo[1,2-f]phenanthridin2-yl)ethan-1-one (2j).

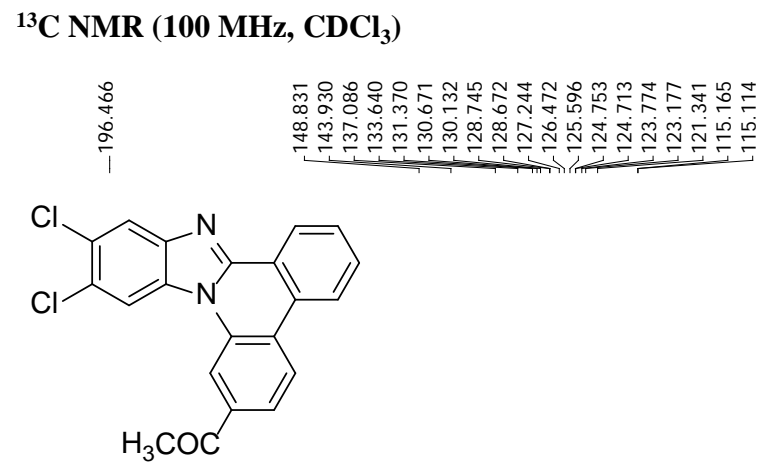

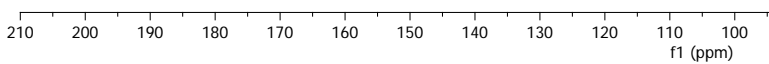

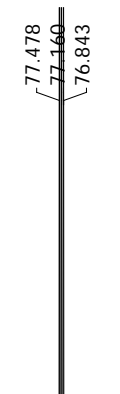

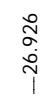

Figure S73. ${ }^{13} \mathrm{C}$ NMR spectrum of 1-(11,12-Dichlorobenzo[4,5]imidazo[1,2-f]phenanthridin2-yl)ethan-1-one (2j). 


\section{${ }^{1} \mathrm{H}$ NMR (700 MHz, $\mathrm{CDCl}_{3}$ )}

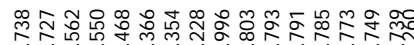

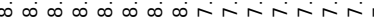

(l)

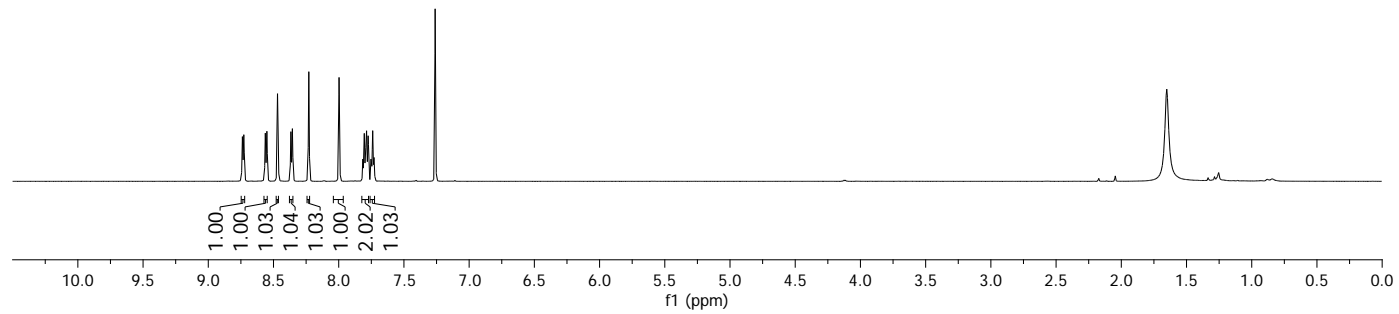

Figure S74. ${ }^{1} \mathrm{H}$ NMR spectrum of 11,12-Dichloro-2-(trifluoromethyl)benzo[4,5]imidazo[1,2f]phenanthridine (2k).

${ }^{13} \mathrm{C}$ NMR (175 MHz, $\mathrm{CDCl}_{3}$ )
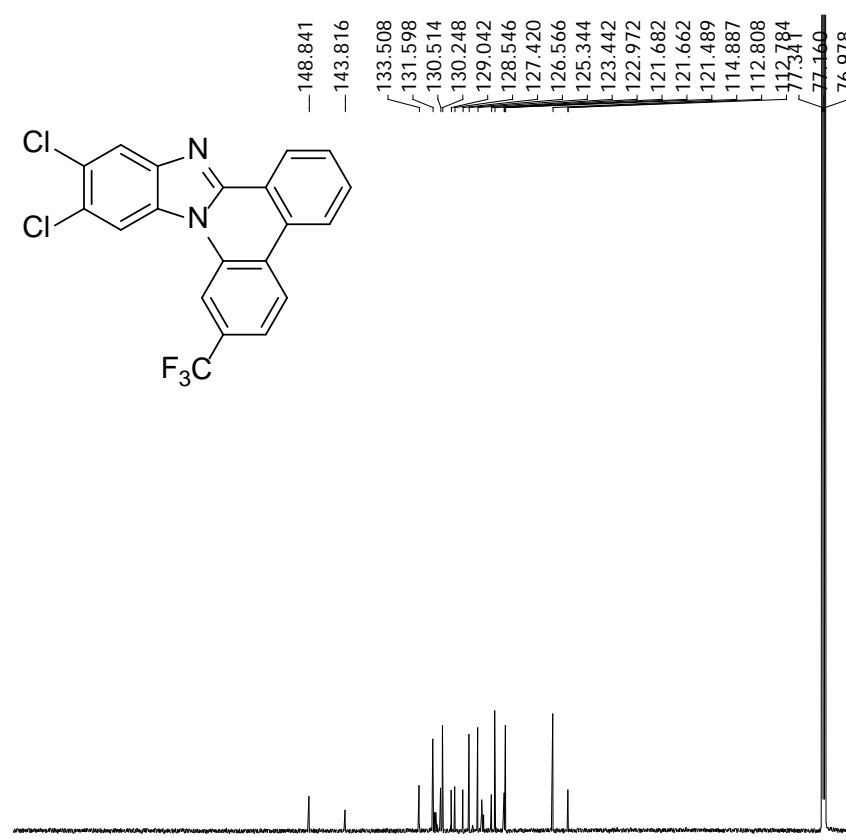

190

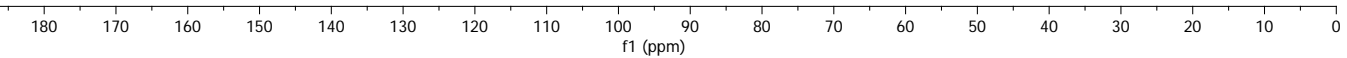

Figure S75. ${ }^{13} \mathrm{C}$ NMR spectrum of 11,12-Dichloro-2(trifluoromethyl)benzo[4,5]imidazo[1,2-f]phenanthridine (2k). 


\section{${ }^{1} \mathrm{H}$ NMR (700 MHz, $\mathrm{CDCl}_{3}$ )}

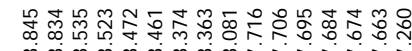

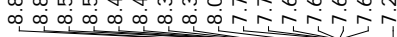<smiles>Cc1cc2nc3c4ccccc4c4ccccc4n3c2cc1C</smiles>

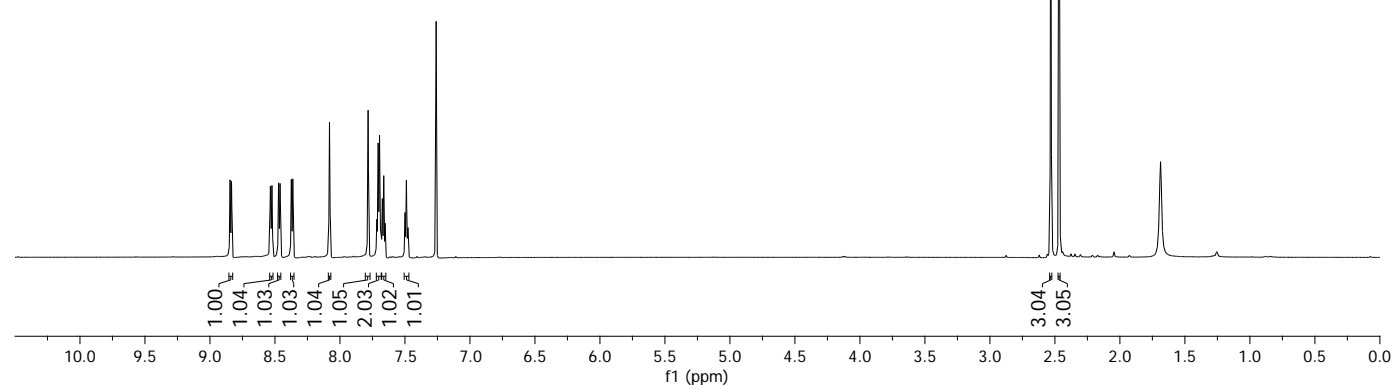

Figure S76. ${ }^{1} \mathrm{H}$ NMR spectrum of 11,12-Dimethylbenzo[4,5]imidazo[1,2-f]phenanthridine (2l).

${ }^{13} \mathrm{C}$ NMR (175 MHz, $\left.\mathrm{CDCl}_{3}\right)$

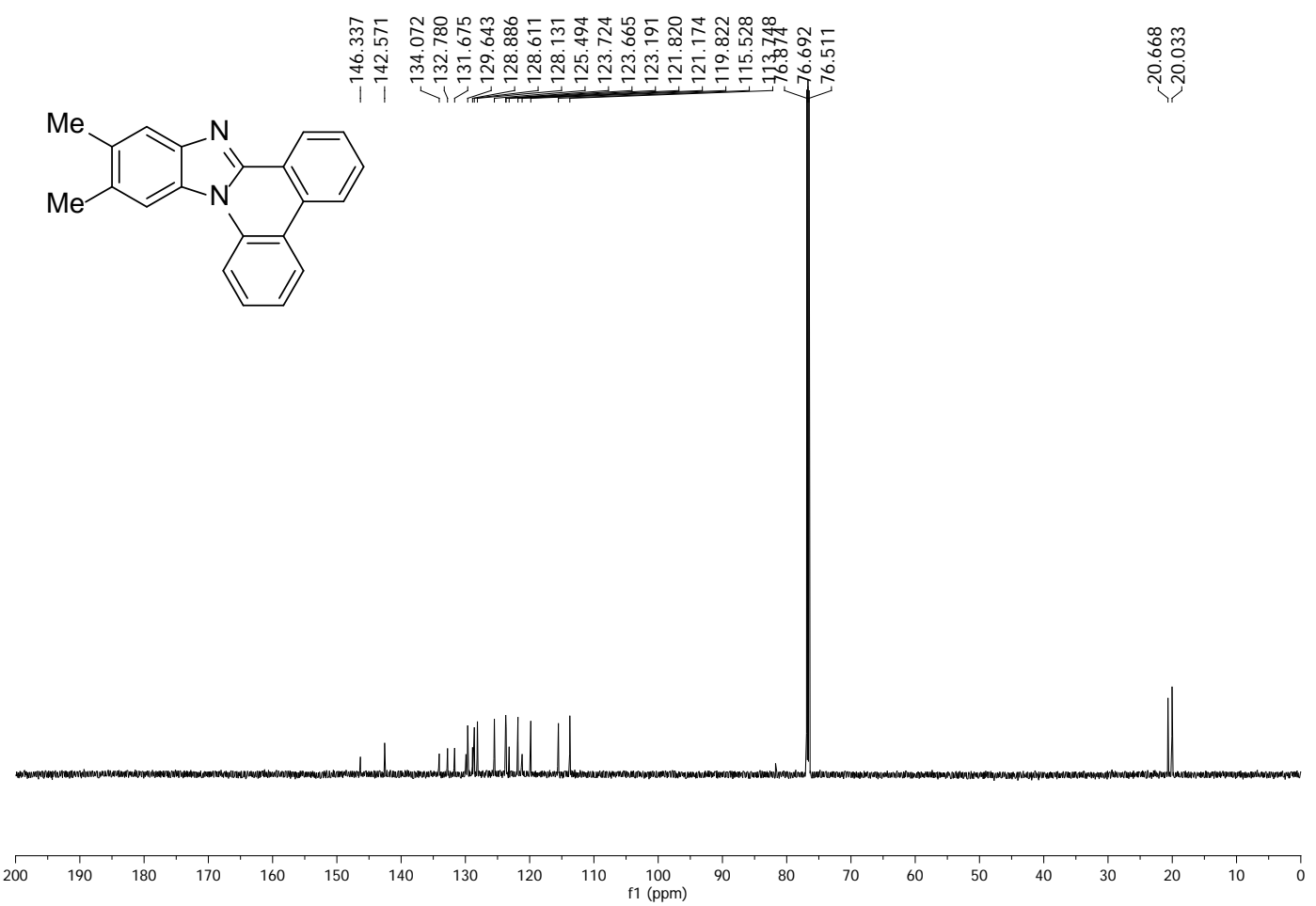

Figure S77. ${ }^{13} \mathrm{C}$ NMR spectrum of 11,12-Dimethylbenzo[4,5]imidazo[1,2-f]phenanthridine (2l). 
${ }^{1}$ H NMR (700 MHz, $\mathrm{CDCl}_{3}$ )

ఇ

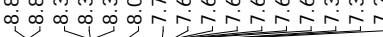

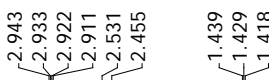

Melll

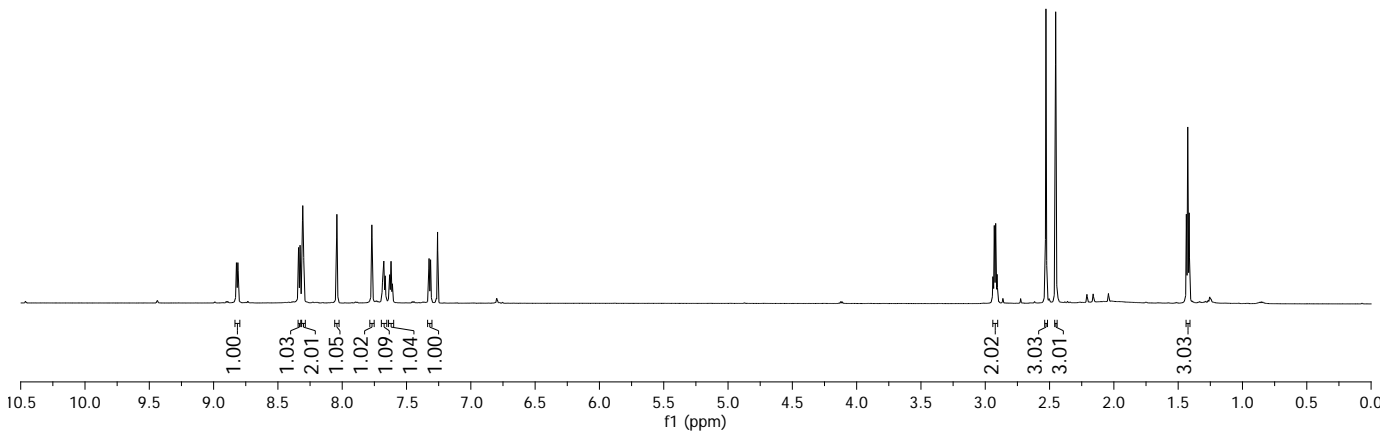

Figure S78. ${ }^{1} \mathrm{H}$ NMR spectrum of 2-Ethyl-11,12-dimethylbenzo[4,5]imidazo[1,2f]phenanthridine (2m).

${ }^{13} \mathrm{C}$ NMR (100 MHz, $\mathrm{CDCl}_{3}$ )<smiles>CCc1ccc2c3ccccc3c3nc4cc(C)c(C)cc4n3c2c1</smiles>

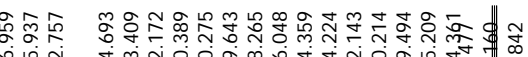

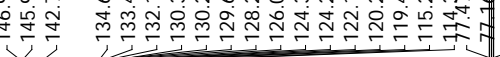

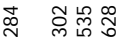

จิ तิำ
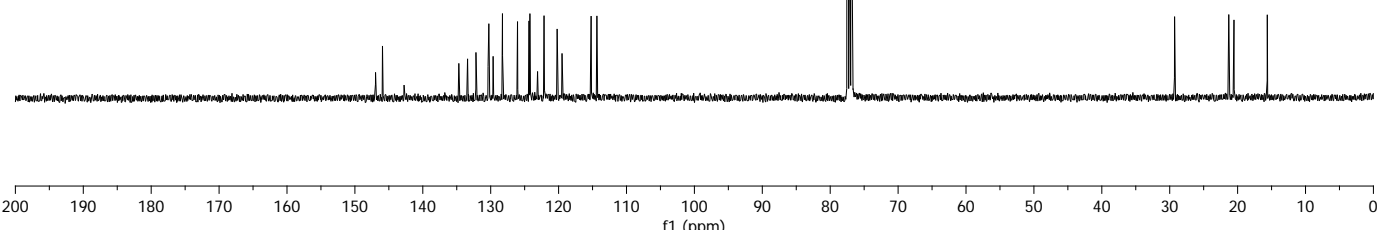

Figure S79. ${ }^{13} \mathrm{C}$ NMR spectrum of 2-Ethyl-11,12-dimethylbenzo[4,5]imidazo[1,2f]phenanthridine (2m). 


\section{${ }^{1} \mathrm{H}$ NMR (700 MHz, $\left.\mathrm{CDCl}_{3}\right)$}

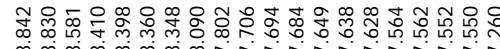

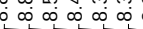<smiles>Cc1cc2nc3c4ccccc4c4ccc(C(C)(C)C)cc4n3c2cc1C</smiles>

${ }^{\mathrm{t}} \mathrm{Bu}$

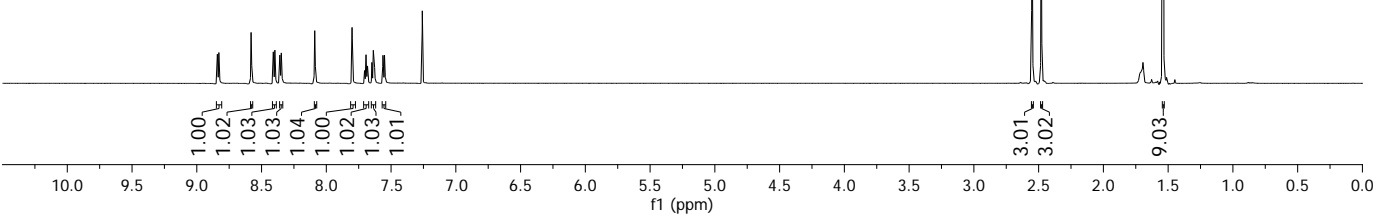

Figure S80. ${ }^{1} \mathrm{H}$ NMR spectrum of 2-(Tert-butyl)-11,12-dimethylbenzo[4,5]imidazo[1,2f]phenanthridine (2n).

${ }^{13} \mathrm{C}$ NMR (175 MHz, $\mathrm{CDCl}_{3}$ )

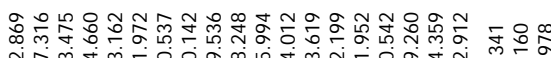

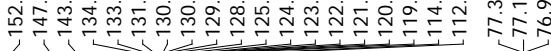<smiles>Cc1cc2nc3c4ccccc4c4ccc(C(C)(C)C)cc4n3c2cc1C</smiles>

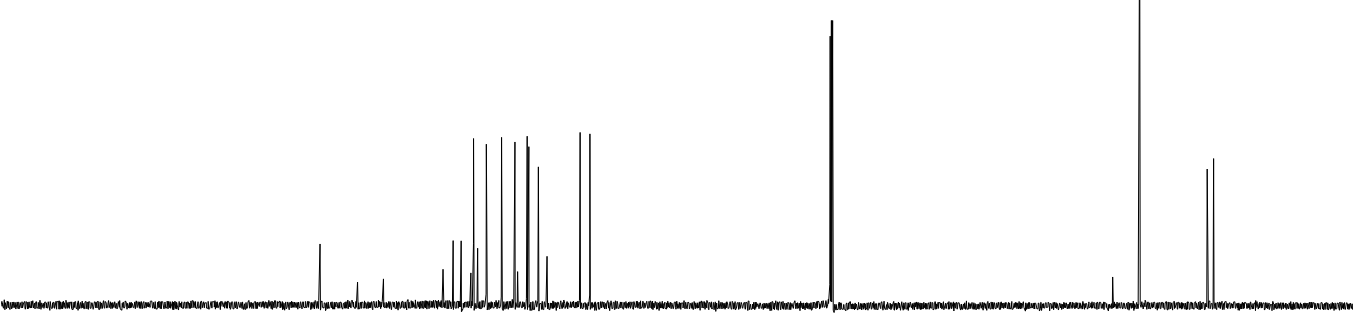

\begin{tabular}{lllllllllllllllllllllllllll}
\hline 200 & 190 & 180 & 170 & 160 & 150 & 140 & 130 & 120 & 110 & 100 & 90 & 80 & 70 & 60 & 50 & 40 & 30 & 20 & 10 & 0
\end{tabular}

Figure S81. ${ }^{13} \mathrm{C}$ NMR spectrum of 2-(Tert-butyl)-11,12-dimethylbenzo[4,5]imidazo[1,2f]phenanthridine (2n). 


\section{${ }^{1} \mathrm{H}$ NMR (700 MHz, $\mathrm{CDCl}_{3}$ )}

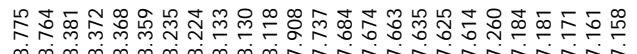

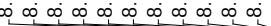<smiles>Cc1cc2nc3c4ccccc4c4ccc(F)cc4n3c2cc1C</smiles>

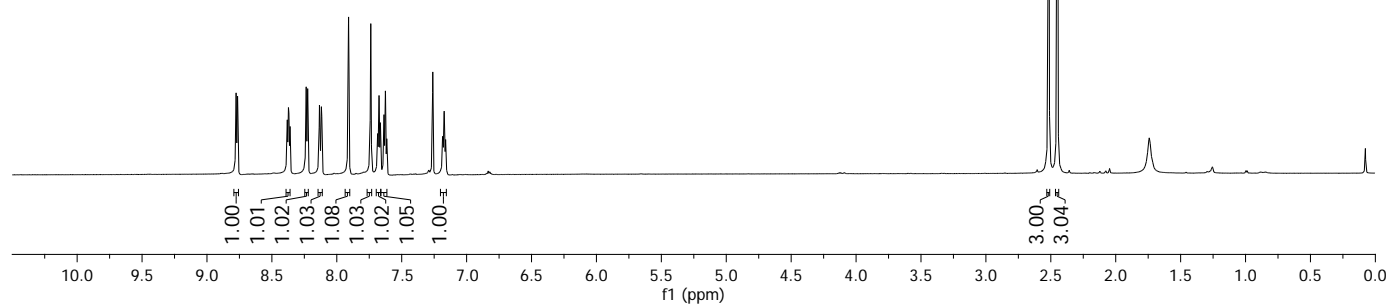

Figure S82. ${ }^{1} \mathrm{H}$ NMR spectrum of 2-Fluoro-11,12-dimethylbenzo[4,5]imidazo[1,2f]phenanthridine (2o).

${ }^{13} \mathrm{C}$ NMR (175 MHz, $\mathrm{CDCl}_{3}$ )
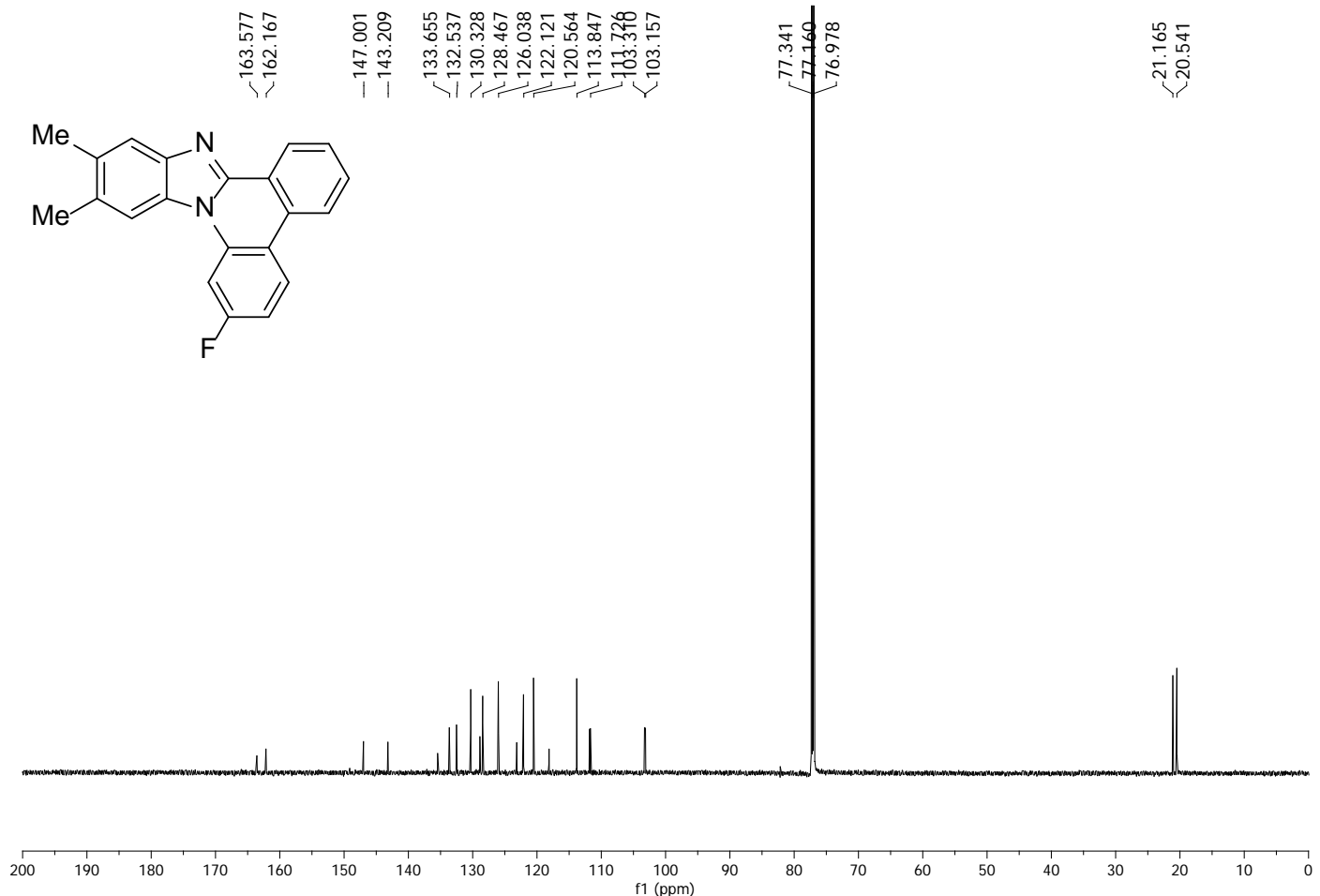

Figure S83. ${ }^{13} \mathrm{C}$ NMR spectrum of 2-Fluoro-11,12-dimethylbenzo[4,5]imidazo[1,2f]phenanthridine (2o). 
${ }^{1} \mathrm{H}$ NMR (700 MHz, $\mathrm{CDCl}_{3}$ )

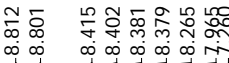

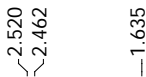<smiles>Cc1cc2nc3c4ccccc4c4cc(Cl)ccc4n3c2cc1C</smiles>

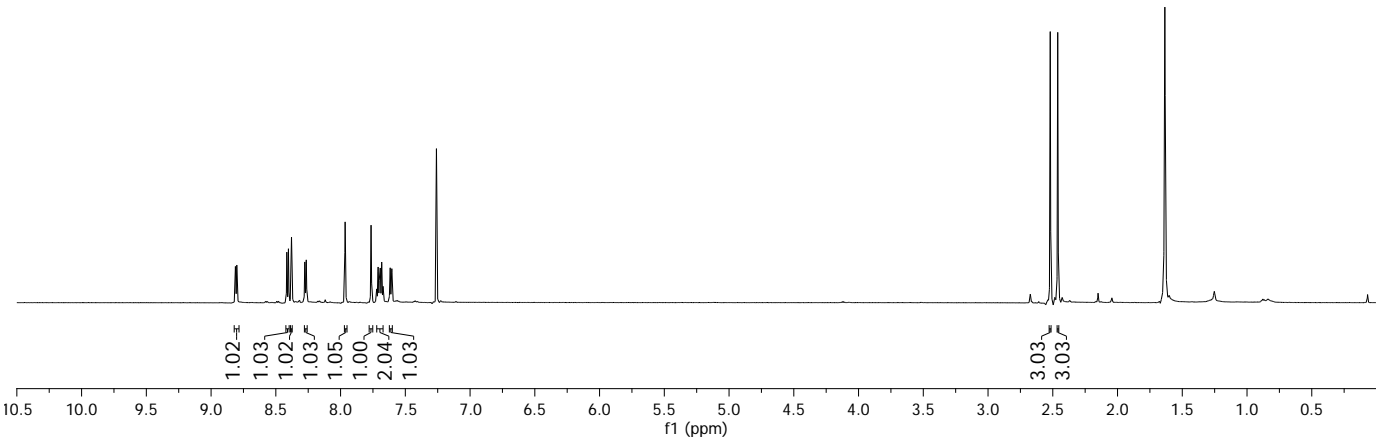

Figure S84. ${ }^{1} \mathrm{H}$ NMR spectrum of 3-Chloro-11,12-dimethylbenzo[4,5]imidazo[1,2f]phenanthridine (2p).

${ }^{13} \mathrm{C}$ NMR (175 MHz, $\mathrm{CDCl}_{3}$

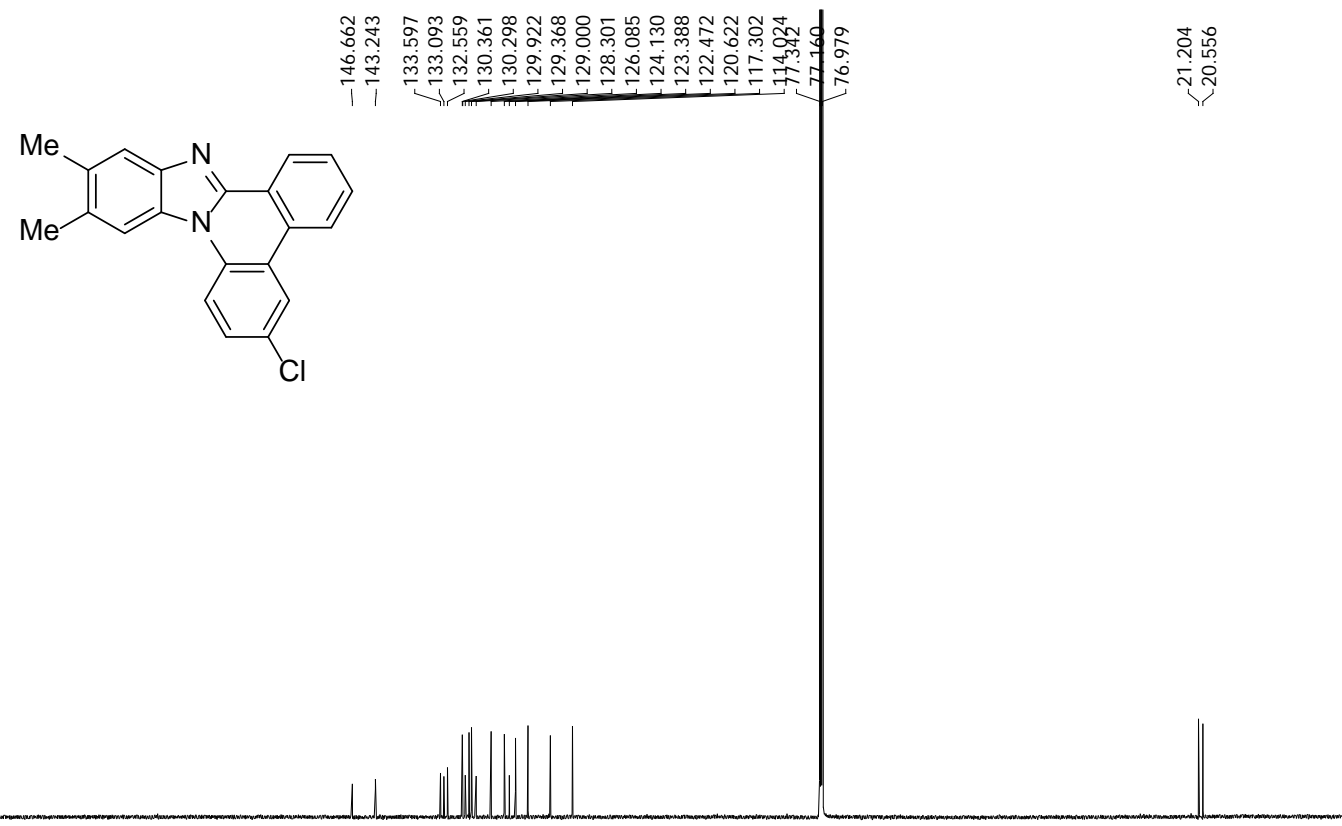

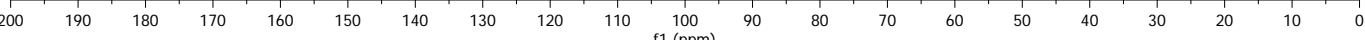

Figure S85. ${ }^{13} \mathrm{C}$ NMR spectrum of 3-Chloro-11,12-dimethylbenzo[4,5]imidazo[1,2f]phenanthridine (2p). 
${ }^{1} \mathrm{H}$ NMR (700 MHz, $\left.\mathrm{CDCl}_{3}\right)$

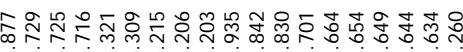

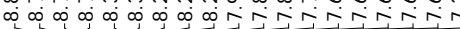

(c)

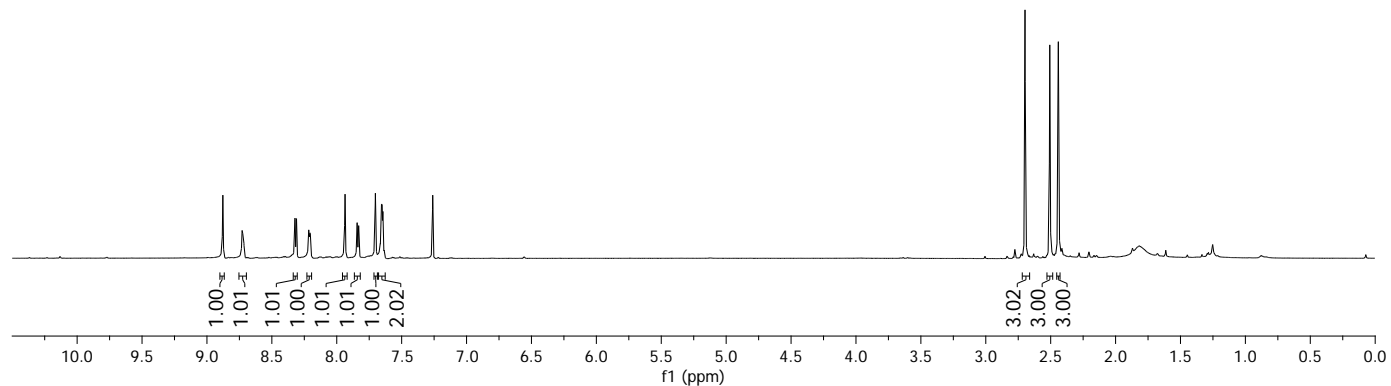

Figure S86. ${ }^{1} \mathrm{H}$ NMR spectrum of 1-(11,12-Dimethylbenzo[4,5]imidazo[1,2-f]phenanthridin2-yl)ethan-1-one (2q).

${ }^{13} \mathrm{C}$ NMR (175 MHz, $\left.\mathrm{CDCl}_{3}\right)$<smiles>CC(=O)c1ccc2c3ccccc3c3nc4cc(C)c(C)cc4n3c2c1</smiles>

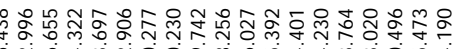

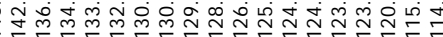

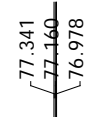

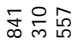

ํㅏㄹ

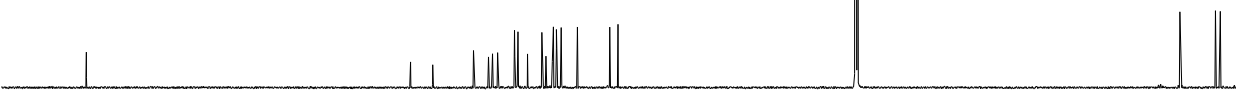

Figure S87. ${ }^{13} \mathrm{C}$ NMR spectrum of 1-(11,12-Dimethylbenzo[4,5]imidazo[1,2f]phenanthridin-2-yl)ethan-1-one (2q). 
${ }^{1} \mathrm{H}$ NMR (400 MHz, $\mathrm{CDCl}_{3}+$ TFA-D)

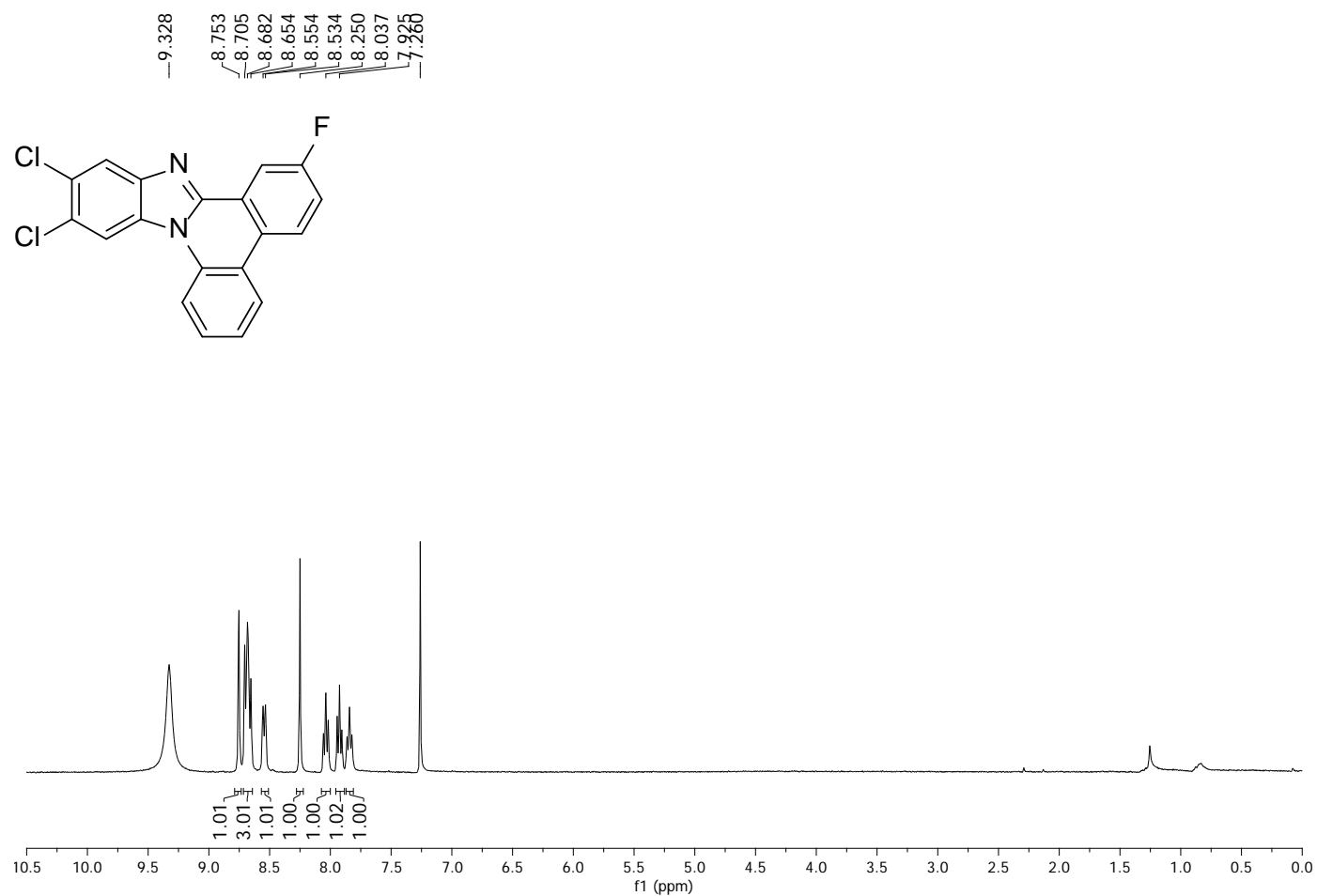

Figure S88. ${ }^{1} \mathrm{H}$ NMR spectrum of 11,12-Dichloro-7-fluorobenzo[4,5]imidazo[1,2f]phenanthridine (2r).

${ }^{13} \mathrm{C}$ NMR (100 MHz, $\mathrm{CDCl}_{3}+$ TFA-D)

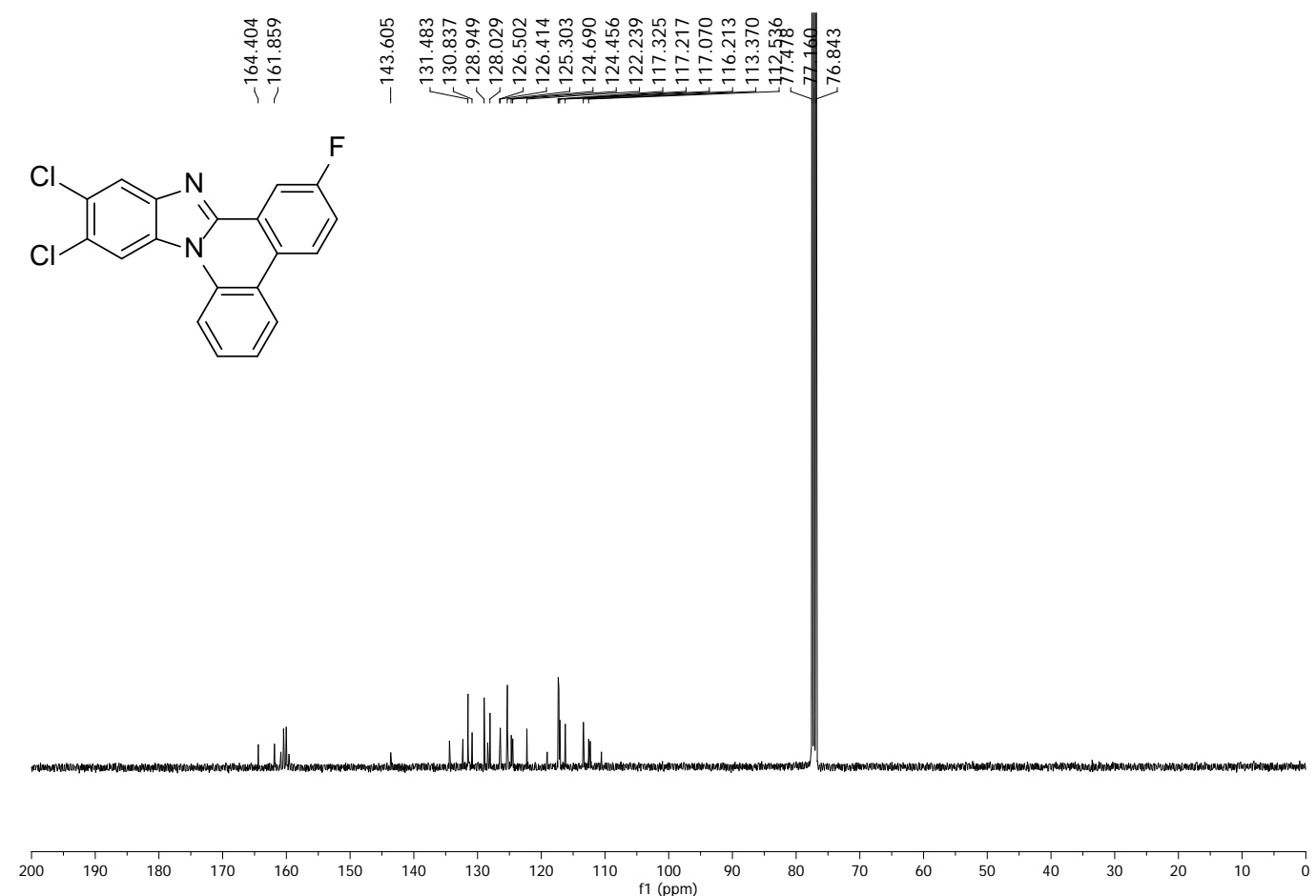

Figure S89. ${ }^{13} \mathrm{C}$ NMR spectrum of 11,12-Dichloro-7-fluorobenzo[4,5]imidazo[1,2f]phenanthridine (2r). 
${ }^{1} \mathrm{H}$ NMR (700 MHz, $\left.\mathrm{CDCl}_{3}\right)$

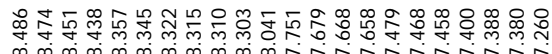

ic

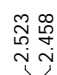<smiles></smiles>

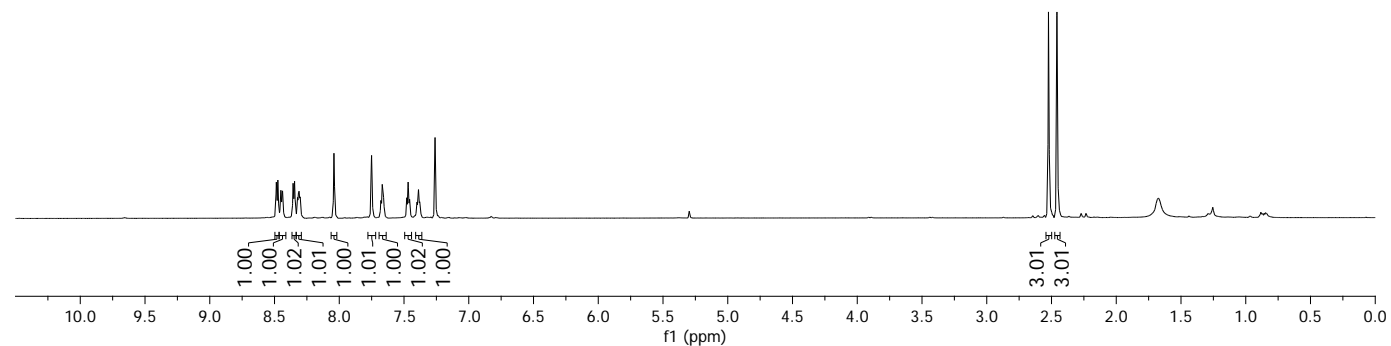

Figure S90. ${ }^{1} \mathrm{H}$ NMR spectrum of 7-Fluoro-11,12-dimethylbenzo[4,5]imidazo[1,2f]phenanthridine (2s).

${ }^{13} \mathrm{C}$ NMR (175 MHz, $\mathrm{CDCl}_{3}$ )

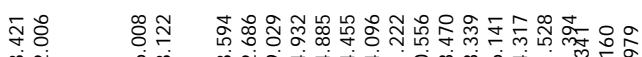

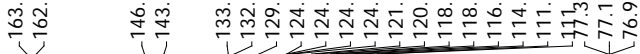

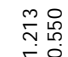

तิ<smiles></smiles>
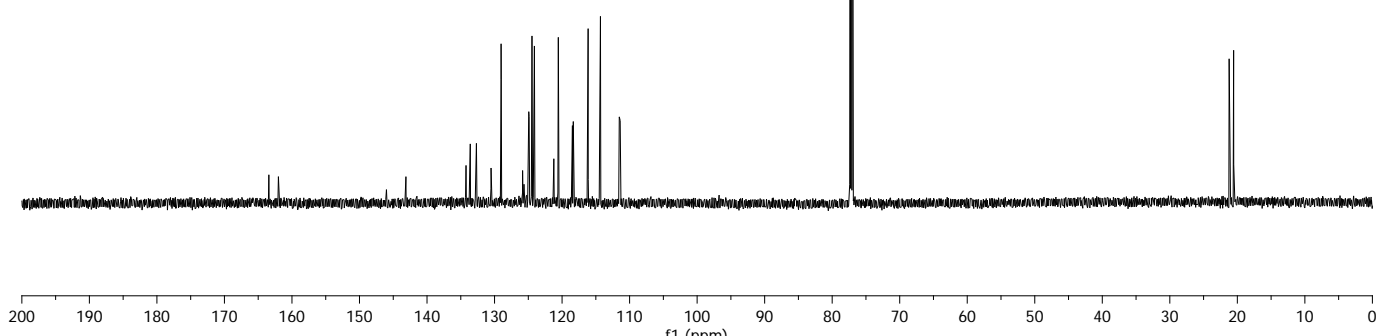

Figure S91. ${ }^{13} \mathrm{C}$ NMR spectrum of 7-Fluoro-11,12-dimethylbenzo[4,5]imidazo[1,2f]phenanthridine (2s). 
${ }^{1}$ H NMR (700 MHz, $\mathrm{CDCl}_{3}$ )

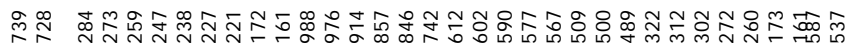

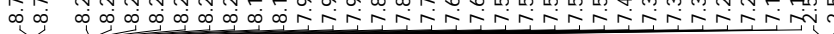

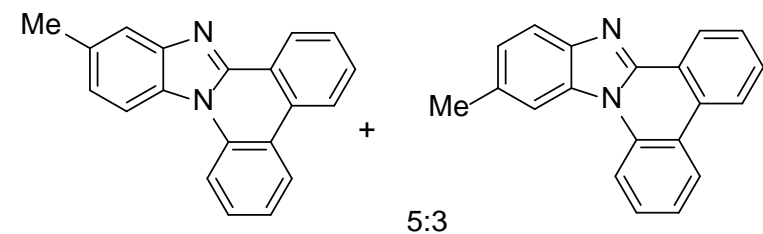

$5: 3$

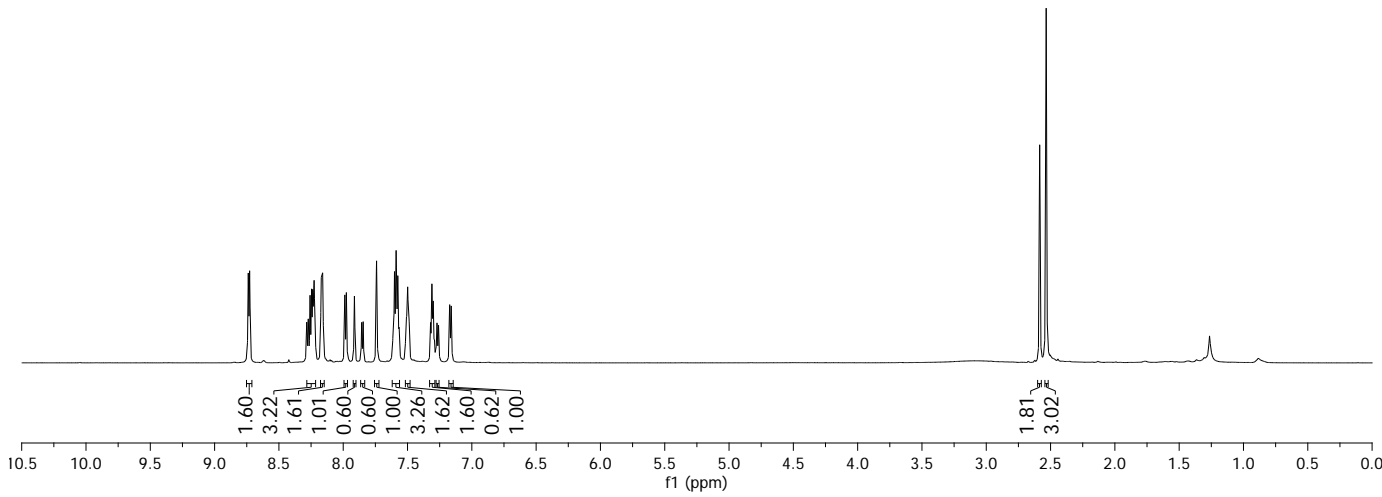

Figure S92. ${ }^{1} \mathrm{H}$ NMR spectrum of 11-methylbenzo[4,5]imidazo[1,2-f]phenanthridine and 12methylbenzo[4,5]imidazo[1,2-f]phenanthridine (2a').

${ }^{13} \mathrm{C}$ NMR (175 MHz, $\mathrm{CDCl}_{3}$ )
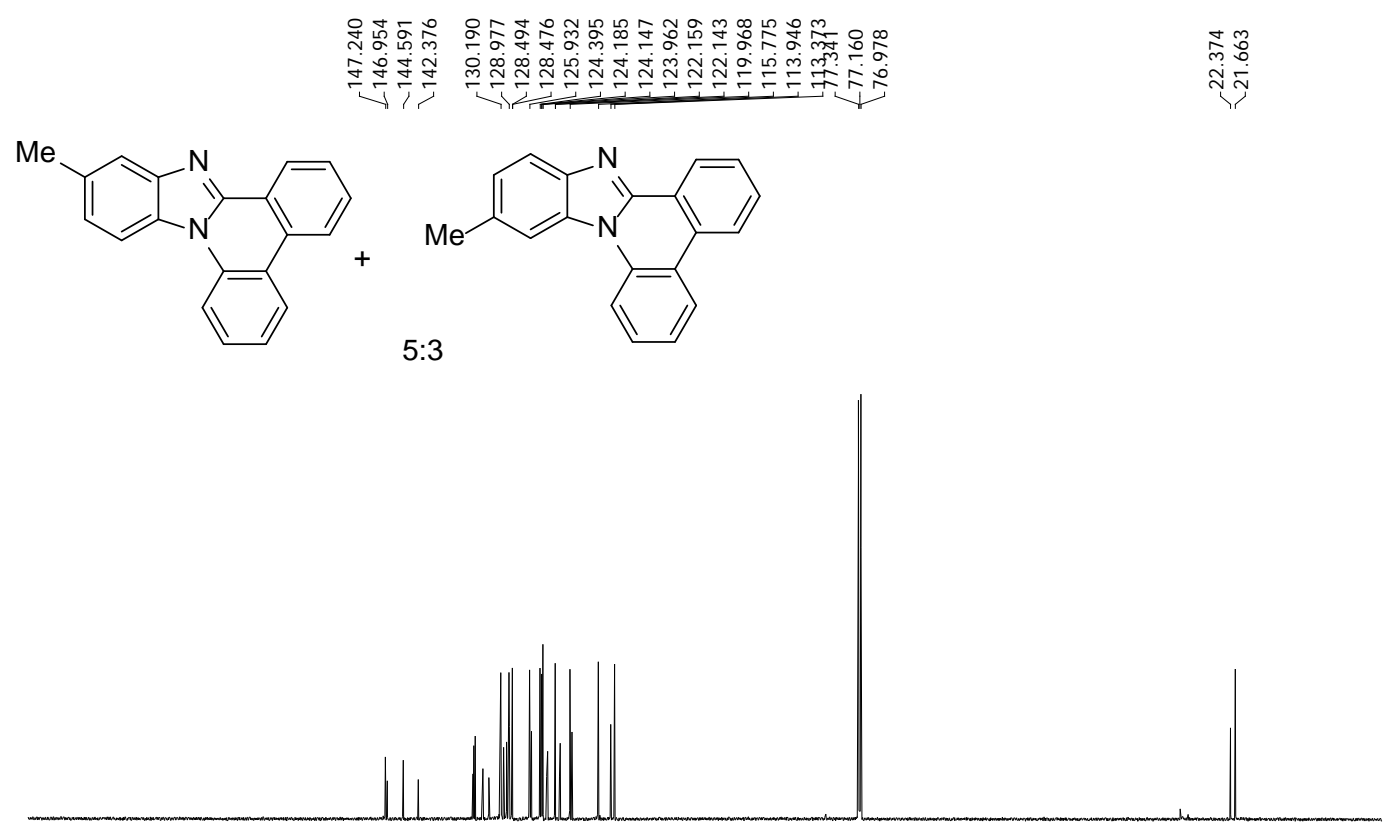

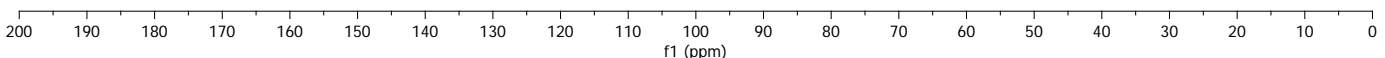

Figure S93. ${ }^{13} \mathrm{C}$ NMR spectrum of 11-methylbenzo[4,5]imidazo[1,2-f]phenanthridine and 12-methylbenzo[4,5]imidazo[1,2-f]phenanthridine (2a'). 
${ }^{1} \mathrm{H}$ NMR (400 MHz, $\left.\mathrm{CDCl}_{3}\right)$

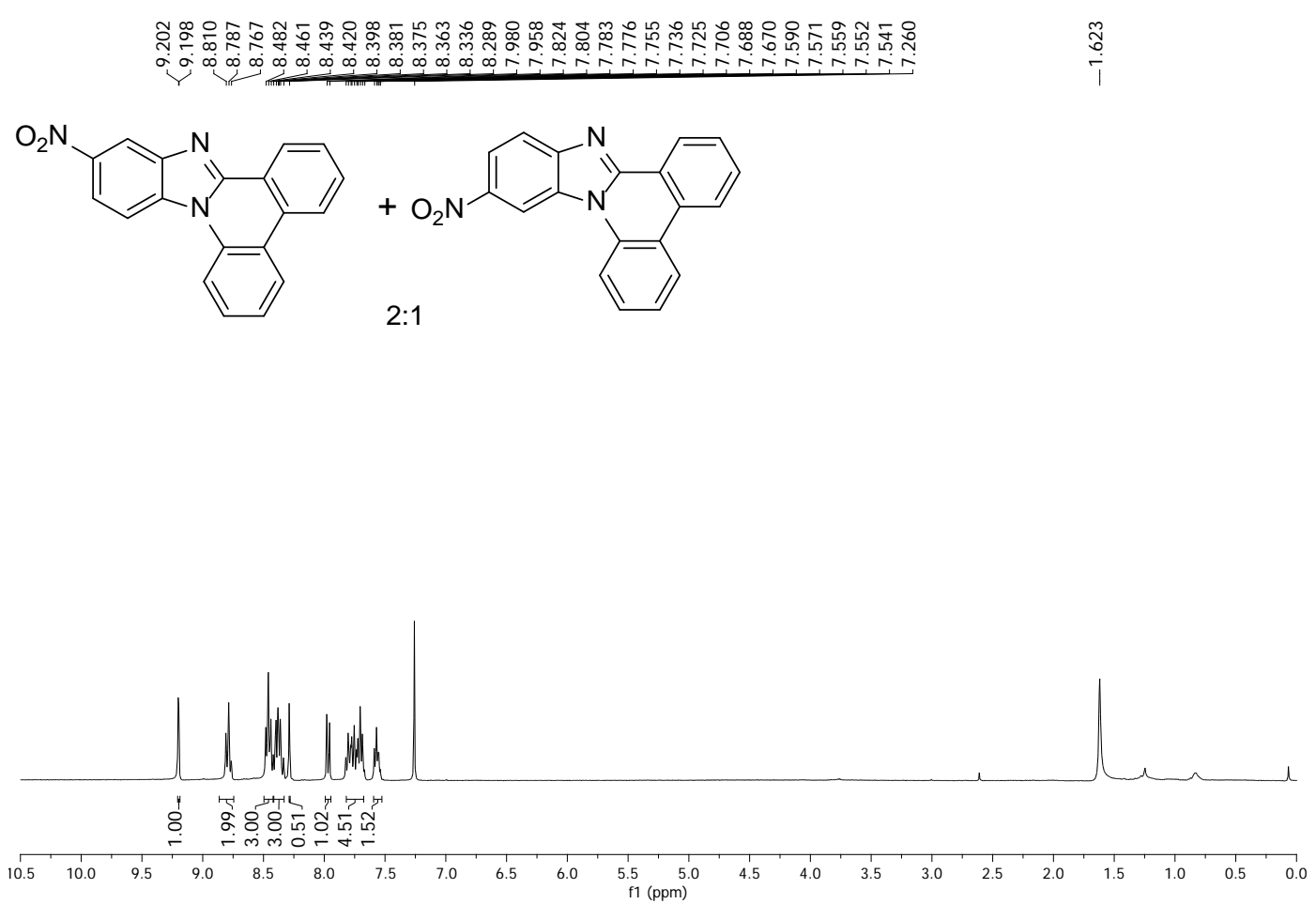

Figure S94. ${ }^{1} \mathrm{H}$ NMR spectrum of 11-nitrobenzo[4,5]imidazo[1,2-f]phenanthridine and 12nitrobenzo[4,5]imidazo[1,2-f]phenanthridine (2b').

${ }^{13} \mathrm{C}$ NMR (100 MHz, $\left.\mathrm{CDCl}_{3}\right)$

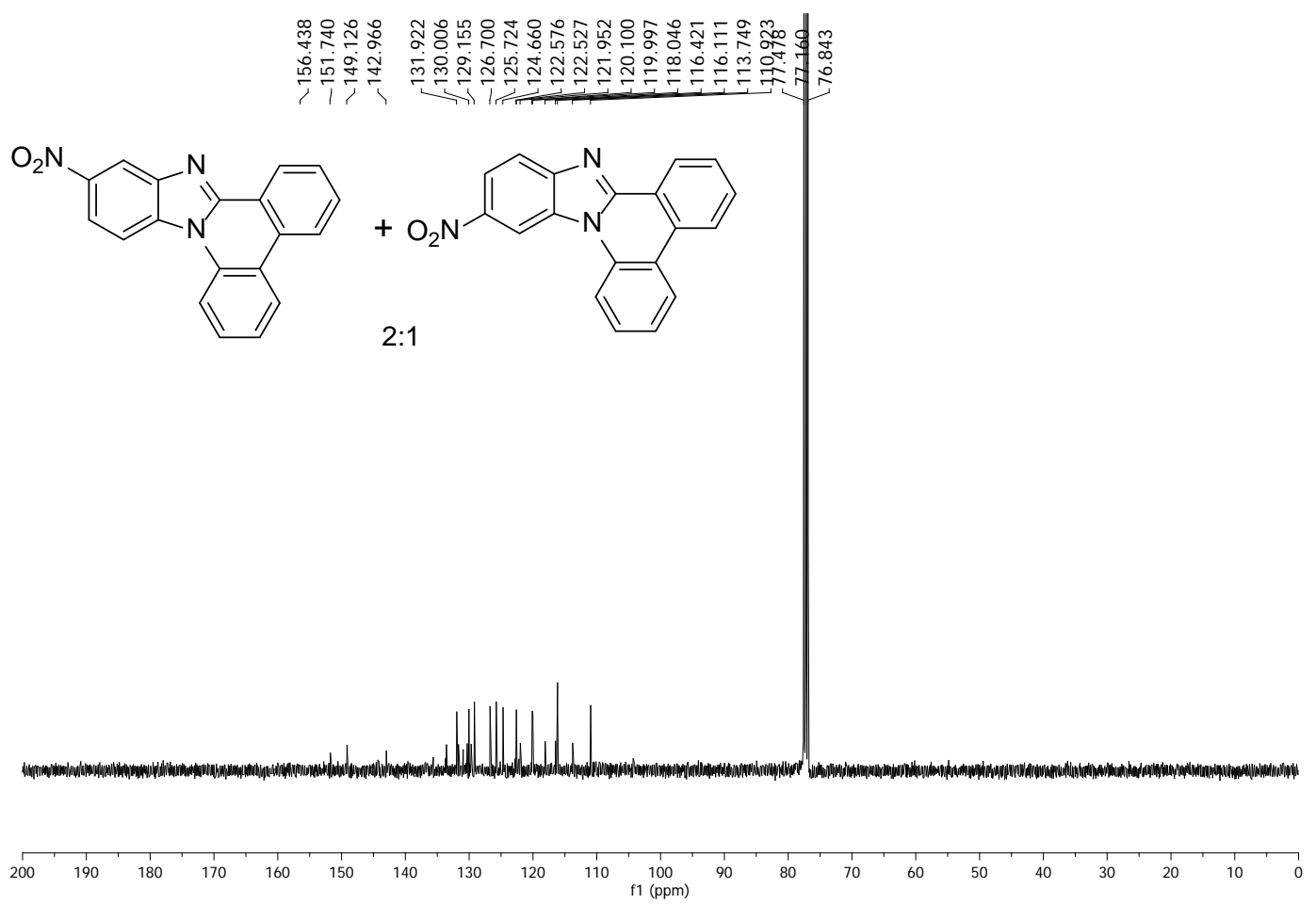

Figure S95. ${ }^{13} \mathrm{C}$ NMR spectrum of 11-nitrobenzo[4,5]imidazo[1,2-f]phenanthridine and 12nitrobenzo[4,5]imidazo[1,2-f]phenanthridine (2b'). 
${ }^{1} \mathrm{H}$ NMR (700 MHz, $\mathrm{CDCl}_{3}$ )

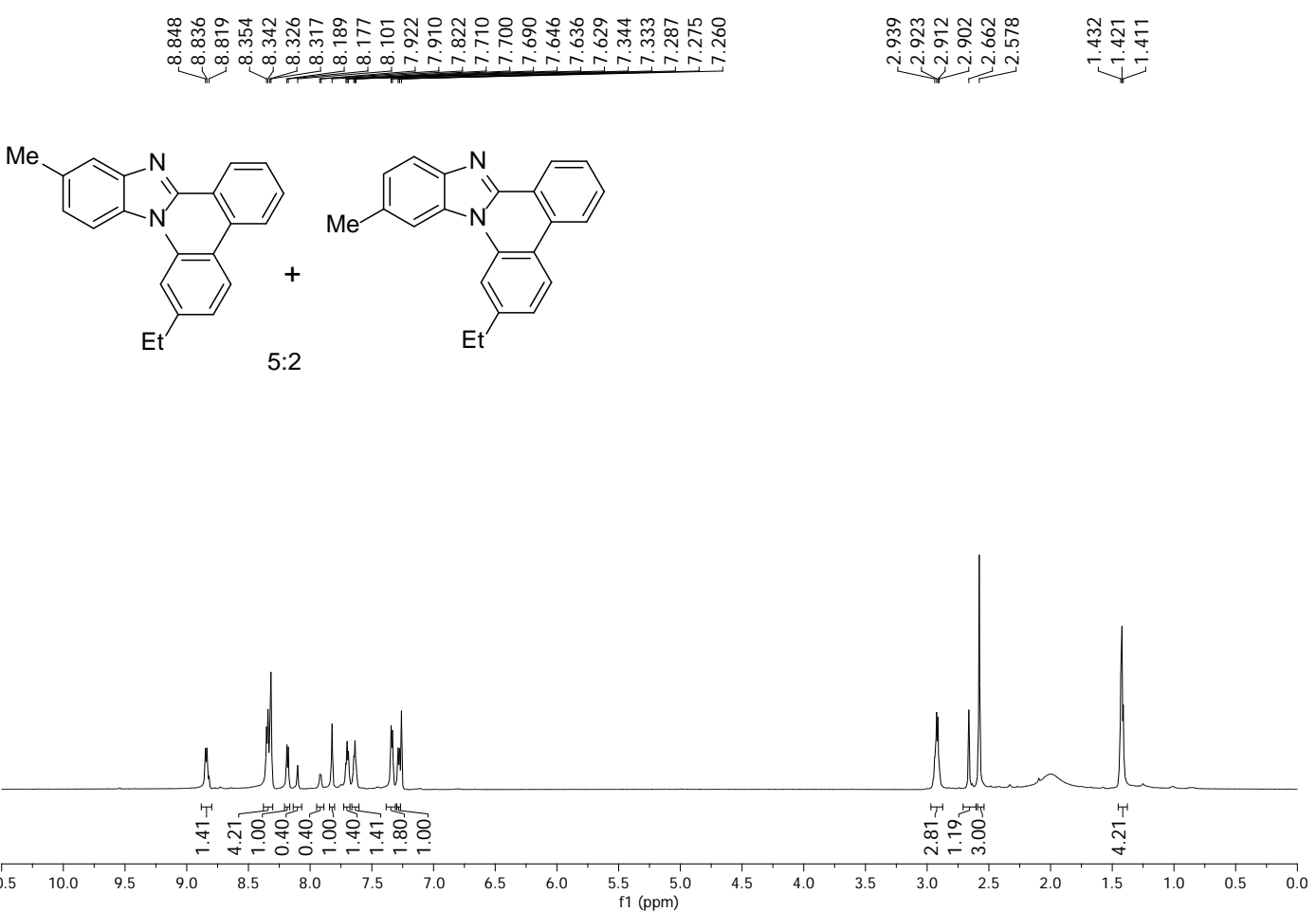

Figure S96. ${ }^{1} \mathrm{H}$ NMR spectrum of 2-Ethyl-11-methylbenzo[4,5]imidazo[1,2-f]phenanthridine and 2-ethyl-12-methylbenzo[4,5]imidazo[1,2-f]phenanthridine (2c').

${ }^{13} \mathrm{C}$ NMR (175 MHz, $\mathrm{CDCl}_{3}$ )

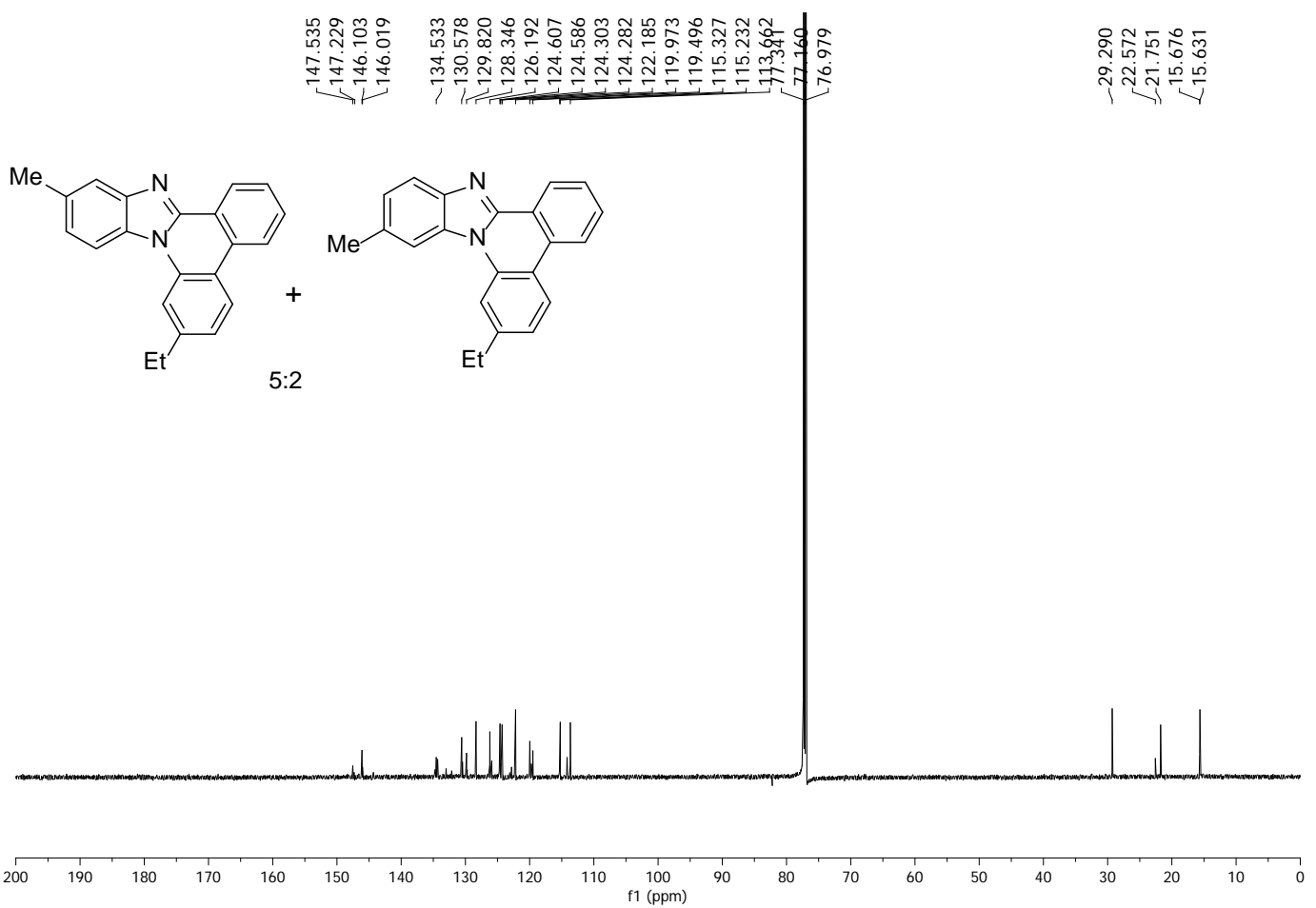

Figure S97. ${ }^{13} \mathrm{C}$ NMR spectrum of 2-Ethyl-11-methylbenzo[4,5]imidazo[1,2f]phenanthridine and 2-ethyl-12-methylbenzo[4,5]imidazo[1,2-f]phenanthridine (2c'). 
${ }^{1} \mathrm{H}$ NMR (400 MHz, $\mathrm{CDCl}_{3}$ )

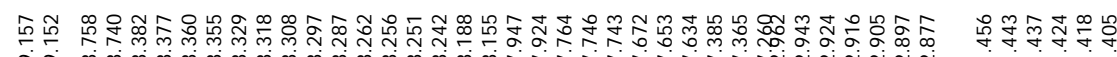

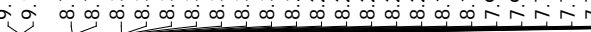

(nt)

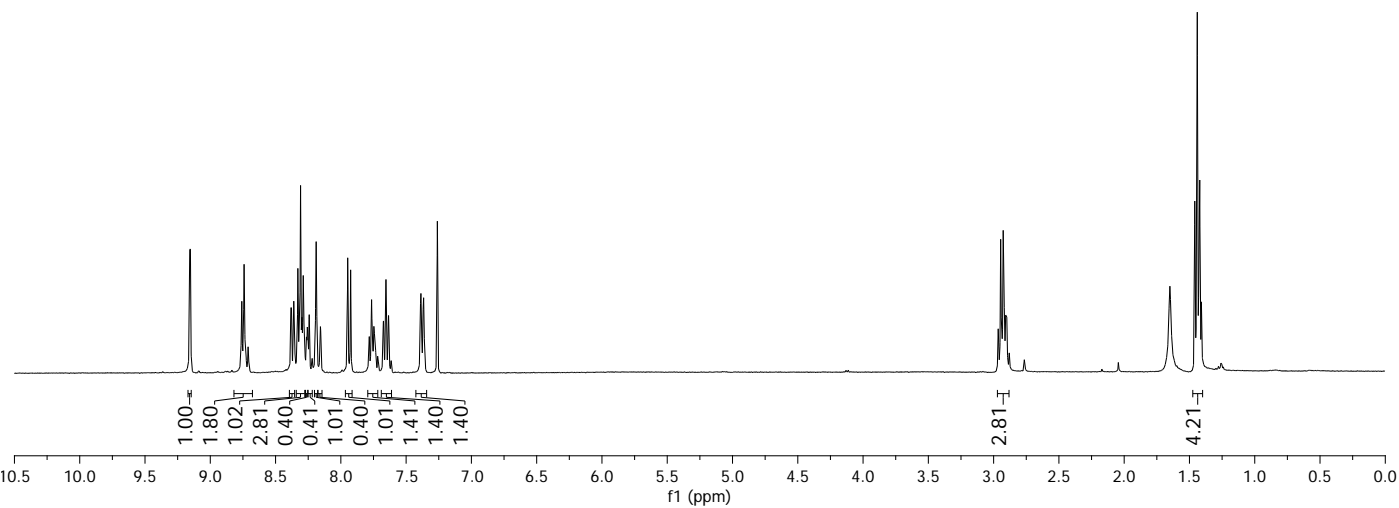

Figure S98. ${ }^{1} \mathrm{H}$ NMR spectrum of 2-Ethyl-11-nitrobenzo[4,5]imidazo[1,2-f]phenanthridine and 2-Ethyl-12-nitrobenzo[4,5]imidazo[1,2-f]phenanthridine (2d').

${ }^{13} \mathrm{C}$ NMR (175 MHz, $\mathrm{CDCl}_{3}$ )

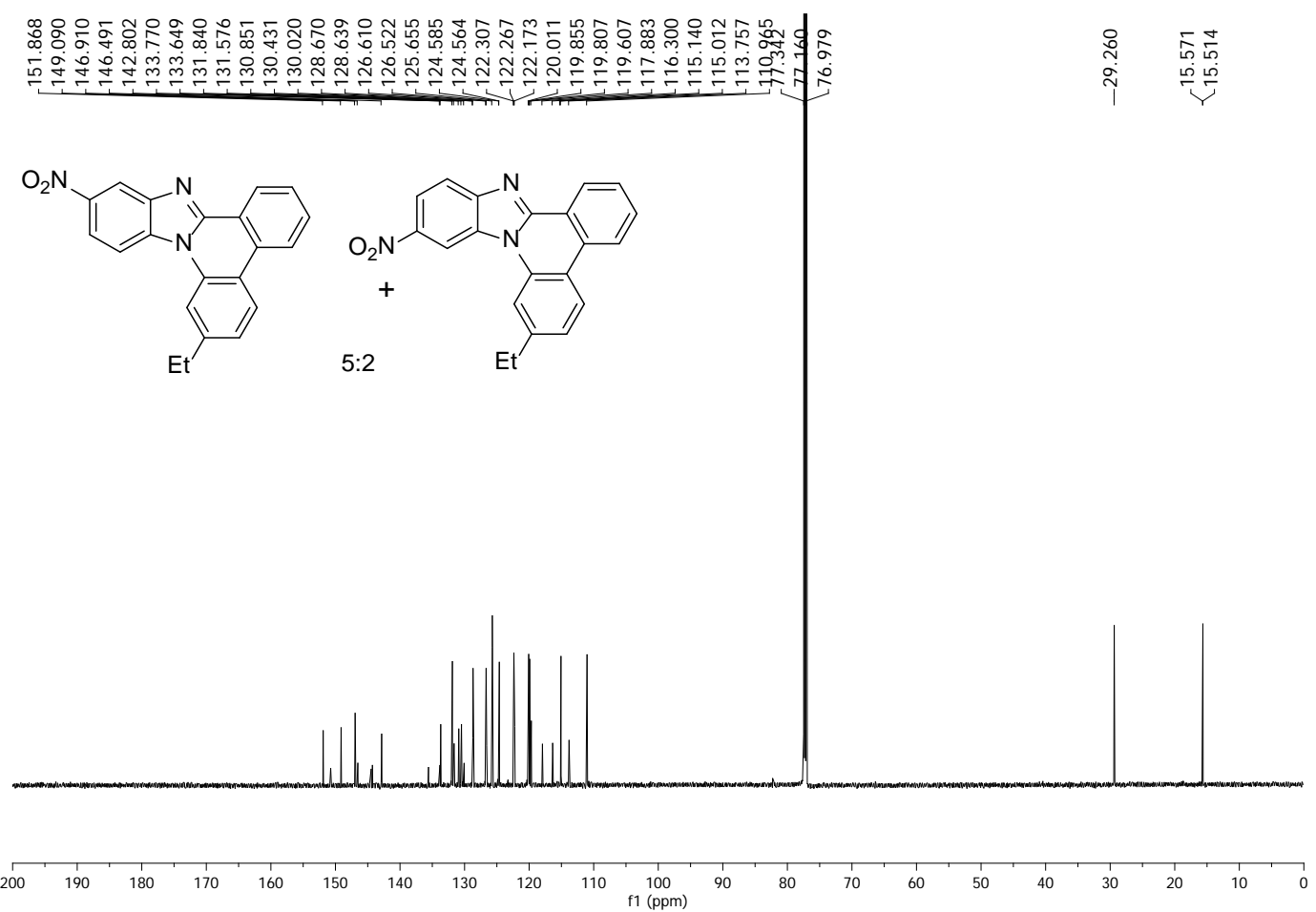

Figure S99. ${ }^{13} \mathrm{C}$ NMR spectrum of 2-Ethyl-11-nitrobenzo[4,5]imidazo[1,2-f]phenanthridine and 2-Ethyl-12-nitrobenzo[4,5]imidazo[1,2-f]phenanthridine (2d'). 


\section{${ }^{1} \mathrm{H}$ NMR (700 MHz, $\mathrm{CDCl}_{3}$ )}

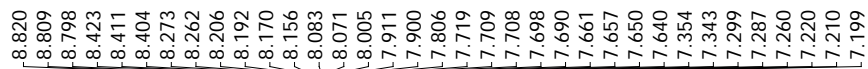
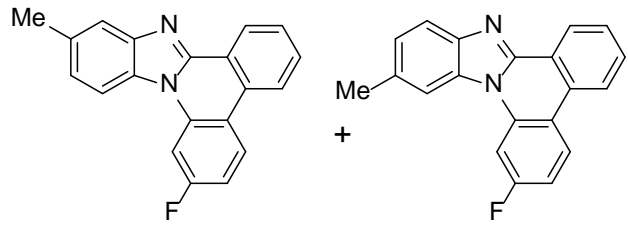

2:1

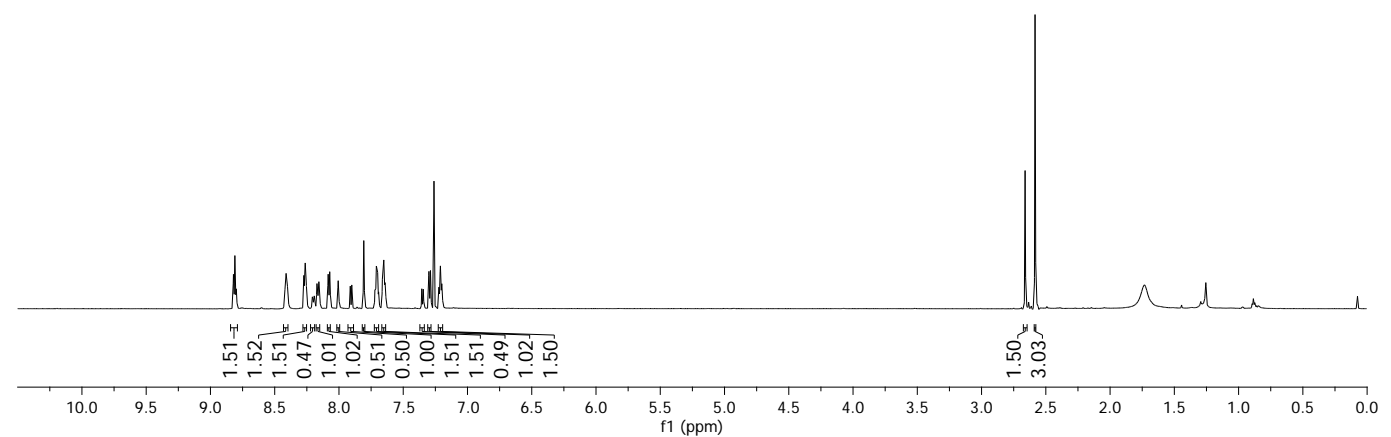

Figure S100. ${ }^{1} \mathrm{H}$ NMR spectrum of 2-Fluoro-11-methylbenzo[4,5]imidazo[1,2f]phenanthridine and 2-Fluoro-12-methylbenzo[4,5]imidazo[1,2-f]phenanthridine (2e').

${ }^{13} \mathrm{C}$ NMR (175 MHz, $\left.\mathrm{CDCl}_{3}\right)$

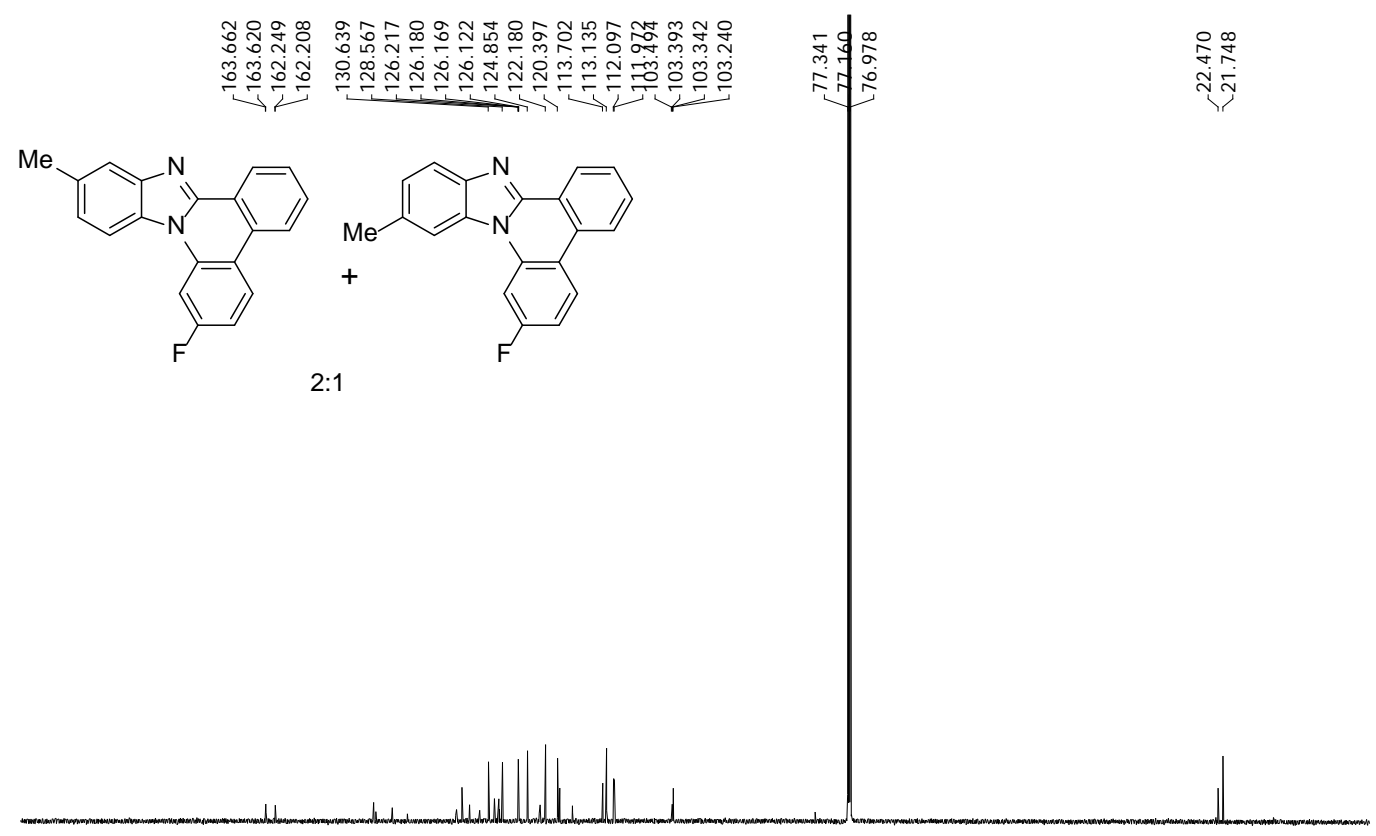

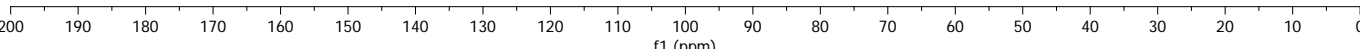

Figure S101. ${ }^{13} \mathrm{C}$ NMR spectrum of 2-Fluoro-11-methylbenzo[4,5]imidazo[1,2f]phenanthridine and 2-Fluoro-12-methylbenzo[4,5]imidazo[1,2-f]phenanthridine (2e'). 
${ }^{1} \mathrm{H}$ NMR (700 MHz, $\mathrm{CDCl}_{3}$ )

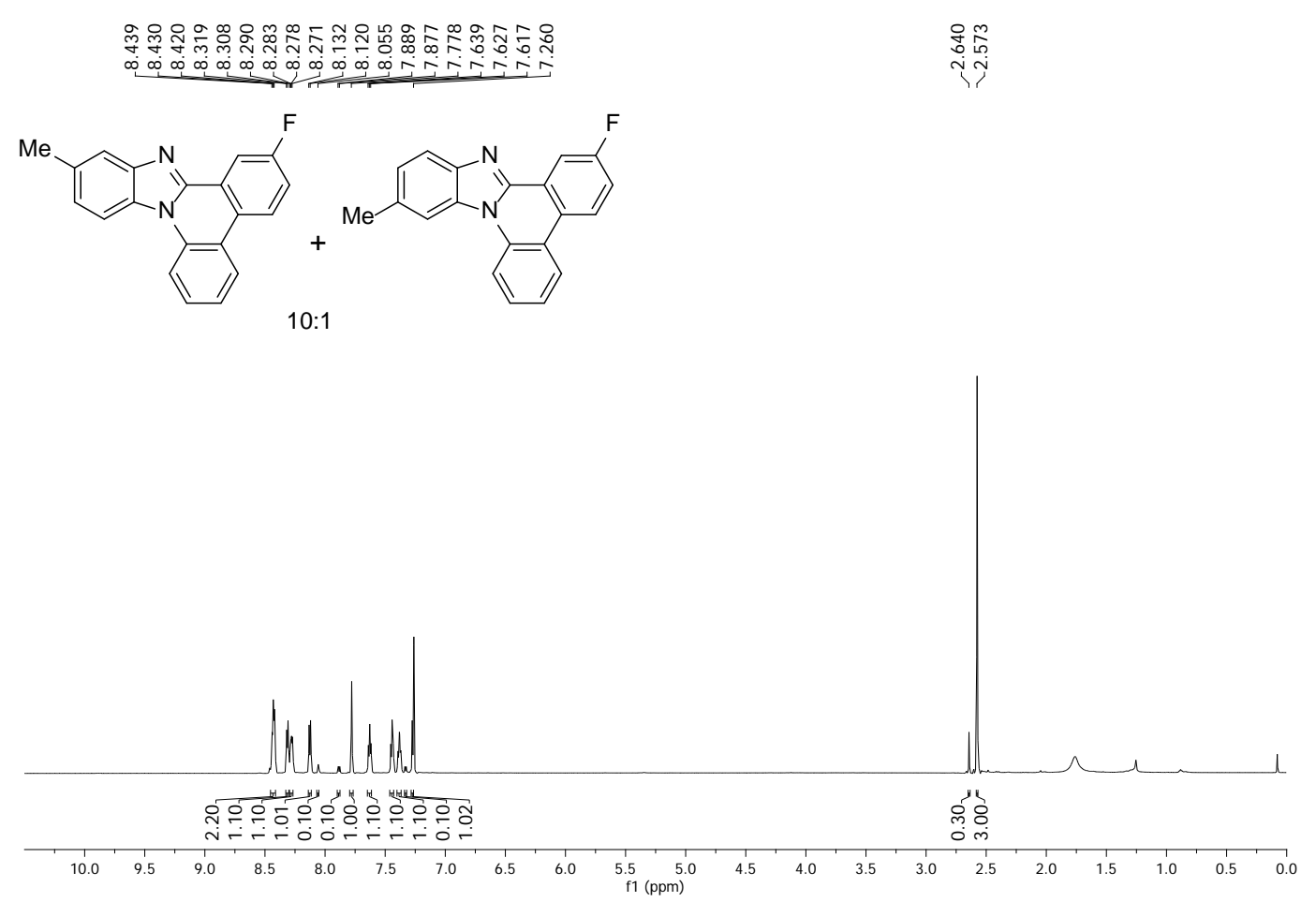

Figure S102. ${ }^{1} \mathrm{H}$ NMR spectrum of 7-Fluoro-11-methylbenzo[4,5]imidazo[1,2f]phenanthridine and 7-Fluoro-12-methylbenzo[4,5]imidazo[1,2-f]phenanthridine (2f').

${ }^{13} \mathrm{C}$ NMR (175 MHz, $\left.\mathrm{CDCl}_{3}\right)$

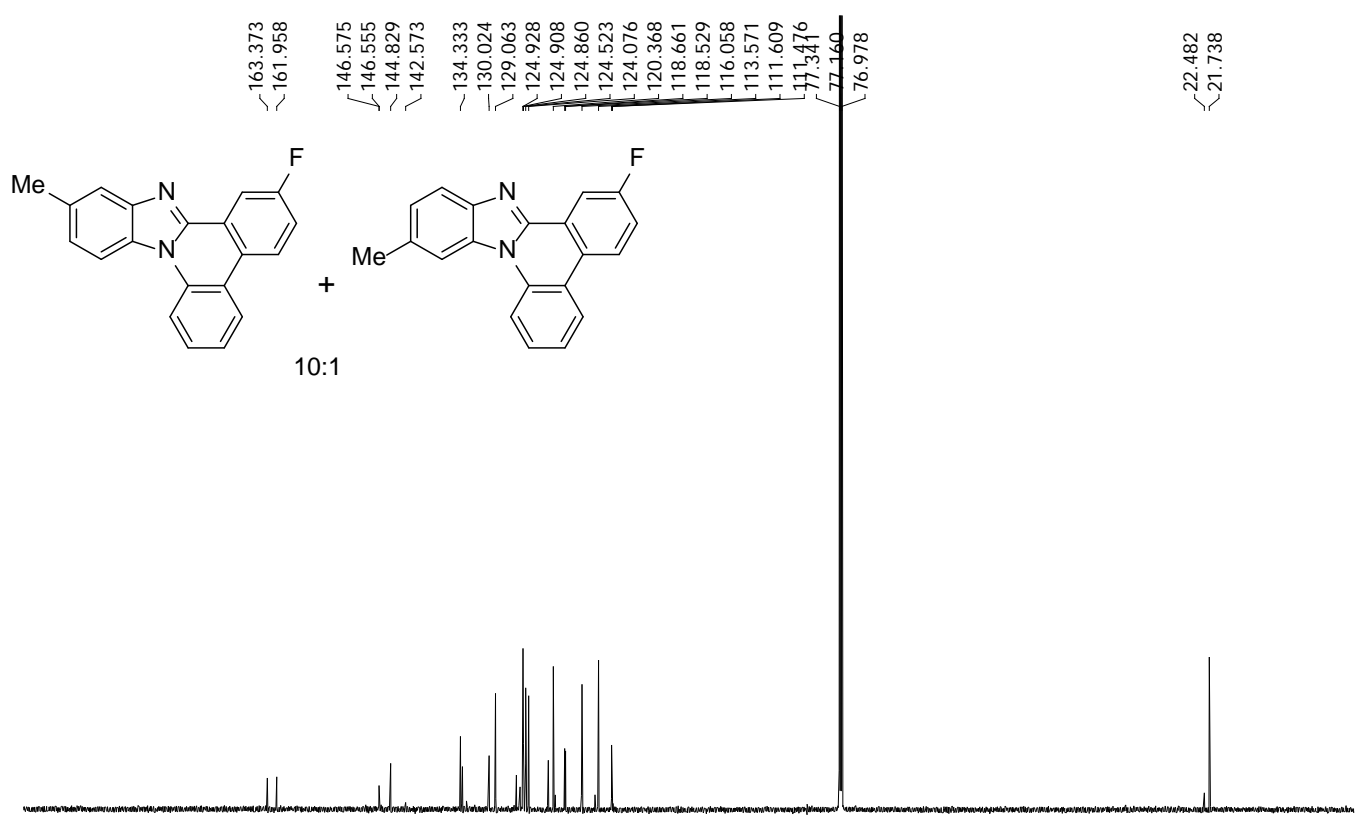

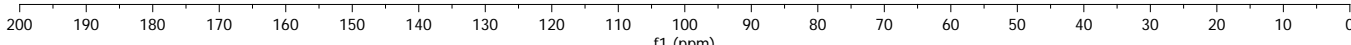

Figure S103. ${ }^{13} \mathrm{C}$ NMR spectrum of 7-Fluoro-11-methylbenzo[4,5]imidazo[1,2f]phenanthridine and 7-Fluoro-12-methylbenzo[4,5]imidazo[1,2-f]phenanthridine (2f'). 
${ }^{1} \mathrm{H}$ NMR (700 MHz, $\left.\mathrm{CDCl}_{3}\right)$

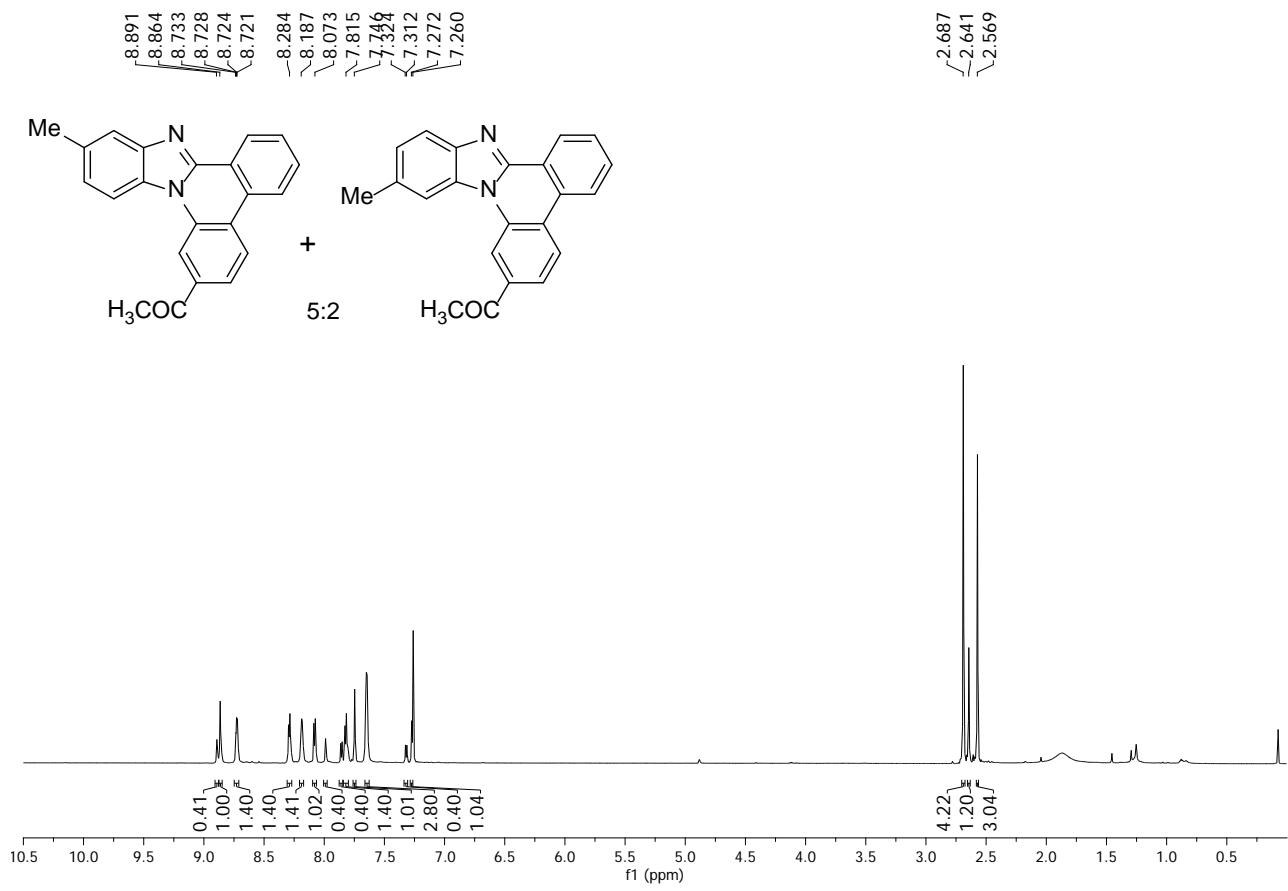

Figure S104. ${ }^{1} \mathrm{H}$ NMR spectrum of 1-(11-Methylbenzo[4,5]imidazo[1,2-f]phenanthridin-2yl)ethan-1-one and 1-(12-Methylbenzo[4,5]imidazo[1,2-f]phenanthridin-2-yl)ethan-1-one $\left(2 g^{\prime}\right)$.

${ }^{13} \mathrm{C}$ NMR (175 MHz, $\mathrm{CDCl}_{3}$ )

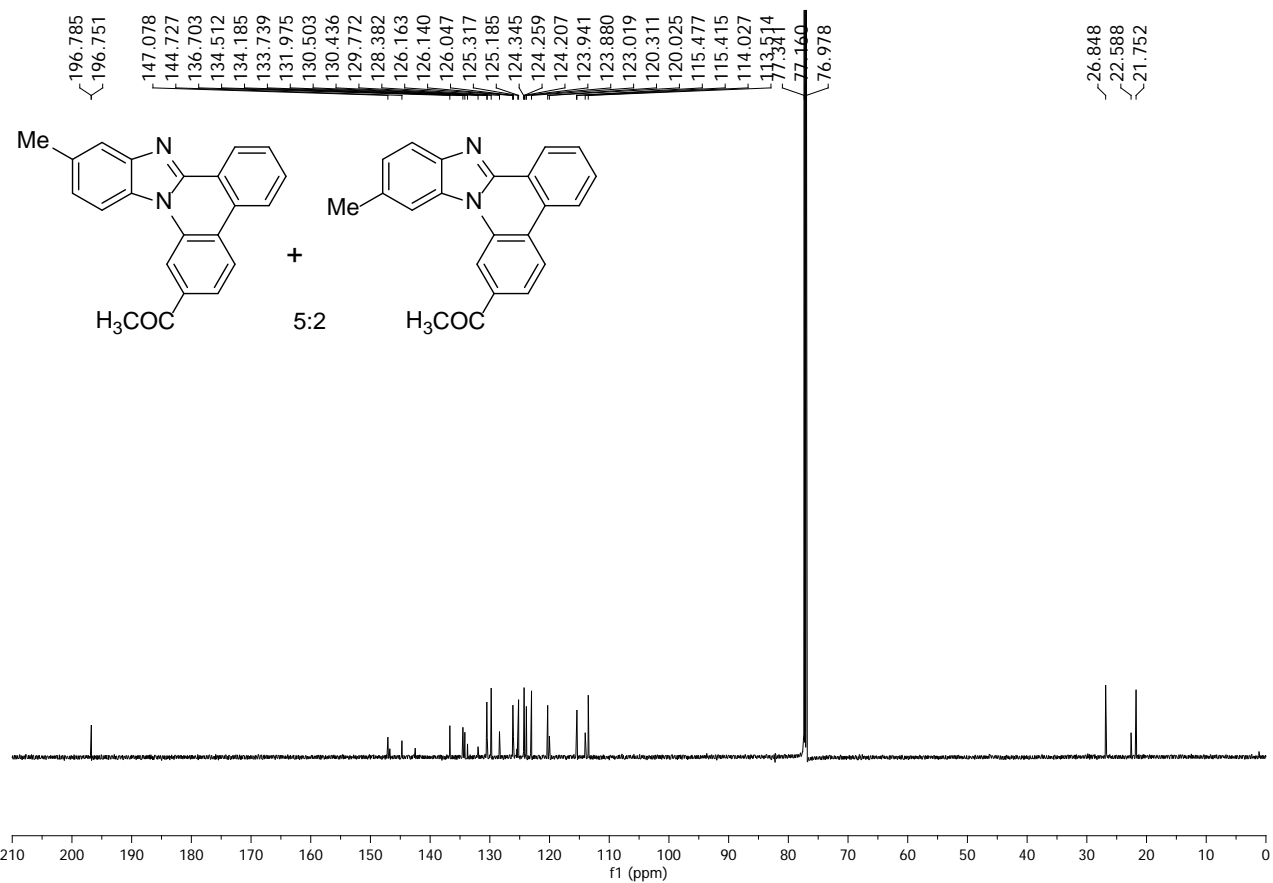

Figure S105. ${ }^{13} \mathrm{C}$ NMR spectrum of 1-(11-Methylbenzo[4,5]imidazo[1,2-f]phenanthridin-2yl)ethan-1-one and 1-(12-Methylbenzo[4,5]imidazo[1,2-f]phenanthridin-2-yl)ethan-1-one (2g'). 


\section{Display Report}

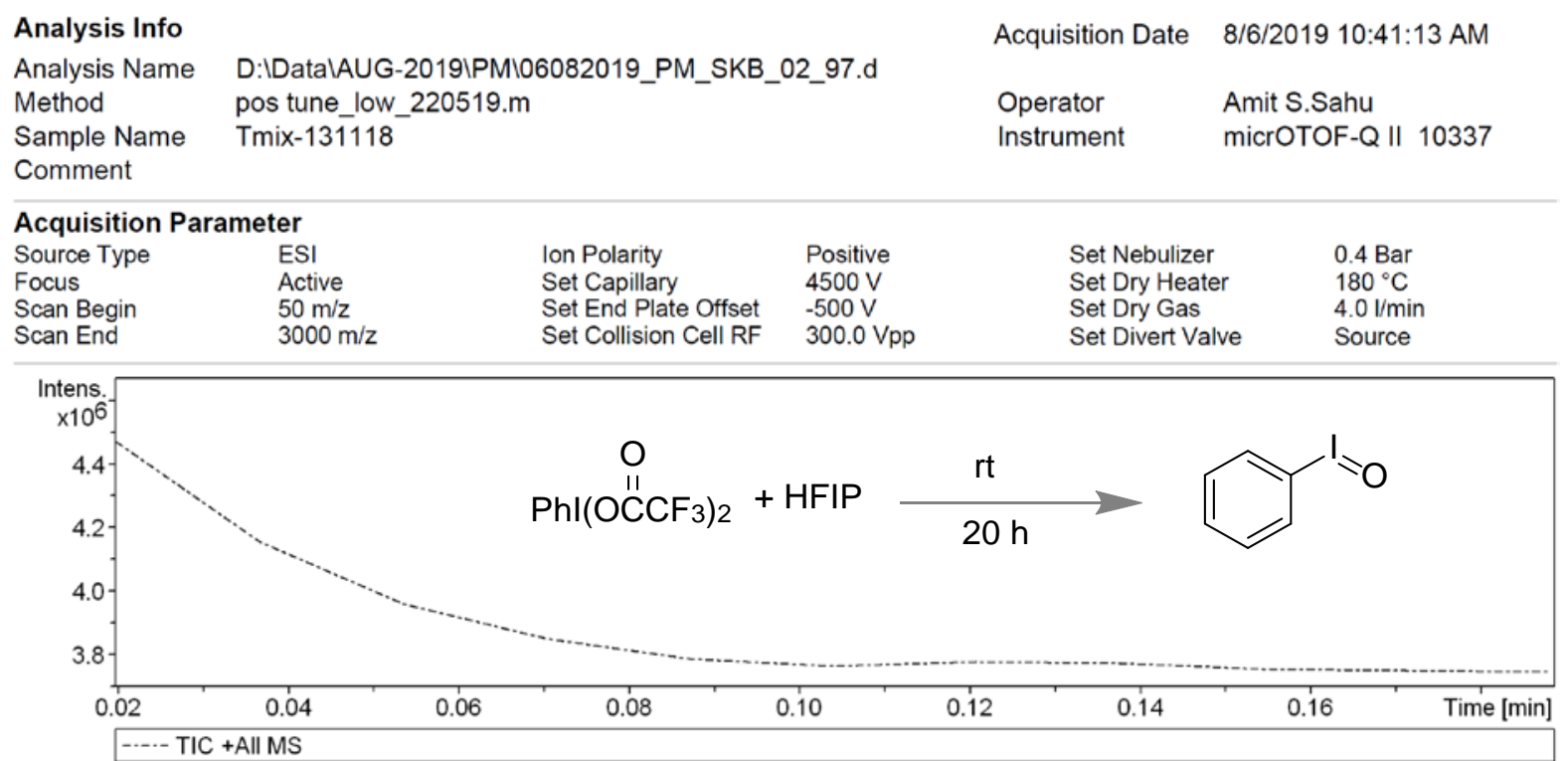
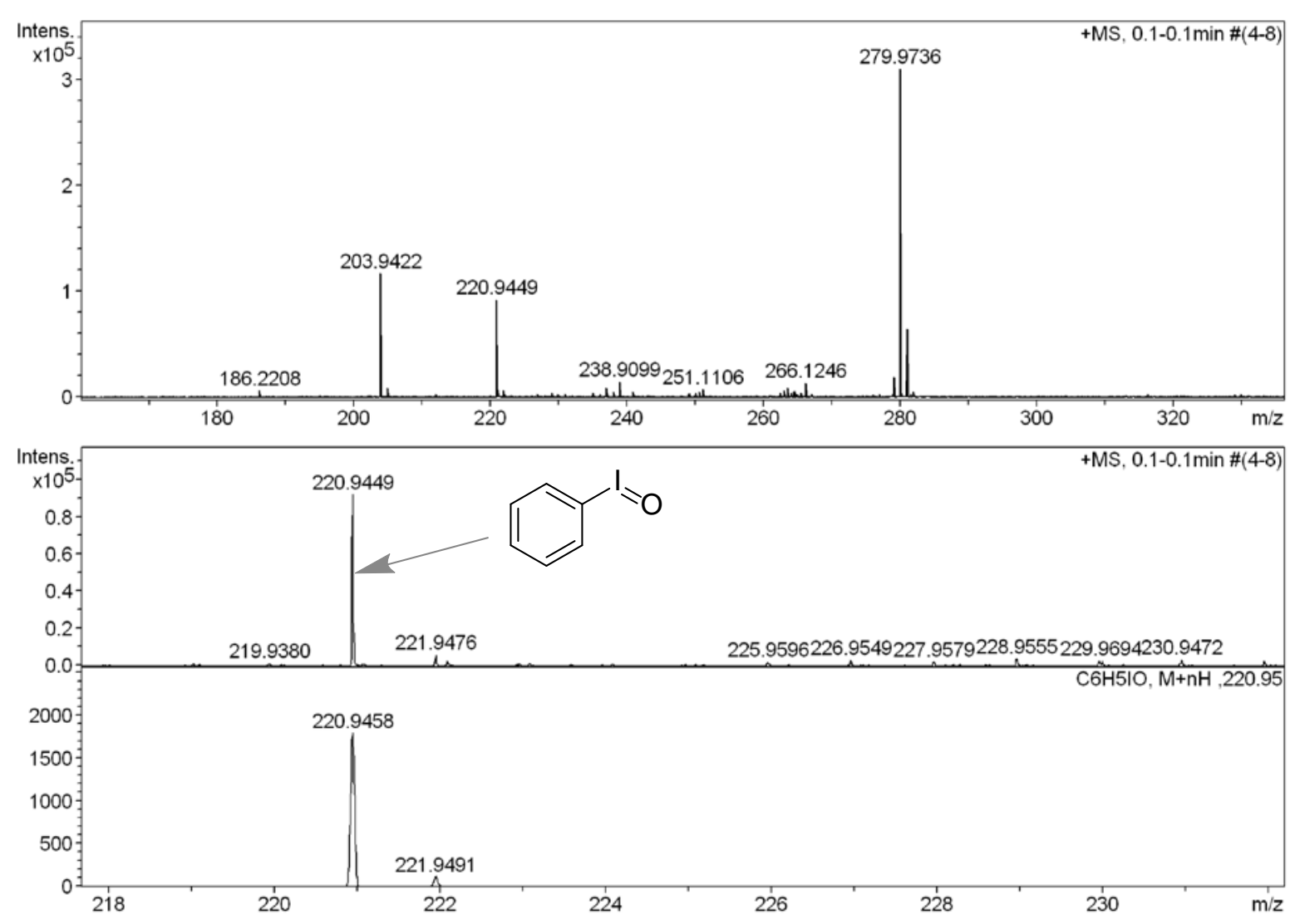

Bruker Compass DataAnalysis 4.0

Figure S106. ESI-MS (HRMS) analysis of crude reaction mixture of PIFA and HFIP. A peak at 220.9449 corresponds to PhIO. 


\section{Display Report}

\section{Analysis Info}

Analysis Name

Method

Sample Name

Comment
Acquisition Date 8/6/2019 10:43:48 AM

D:IData\AUG-2019IPMI06082019_PM_SKB_02_98.d

pos tune low 220519.m

Operator Amit S.Sahu

Instrument micrOTOF-Q || 10337
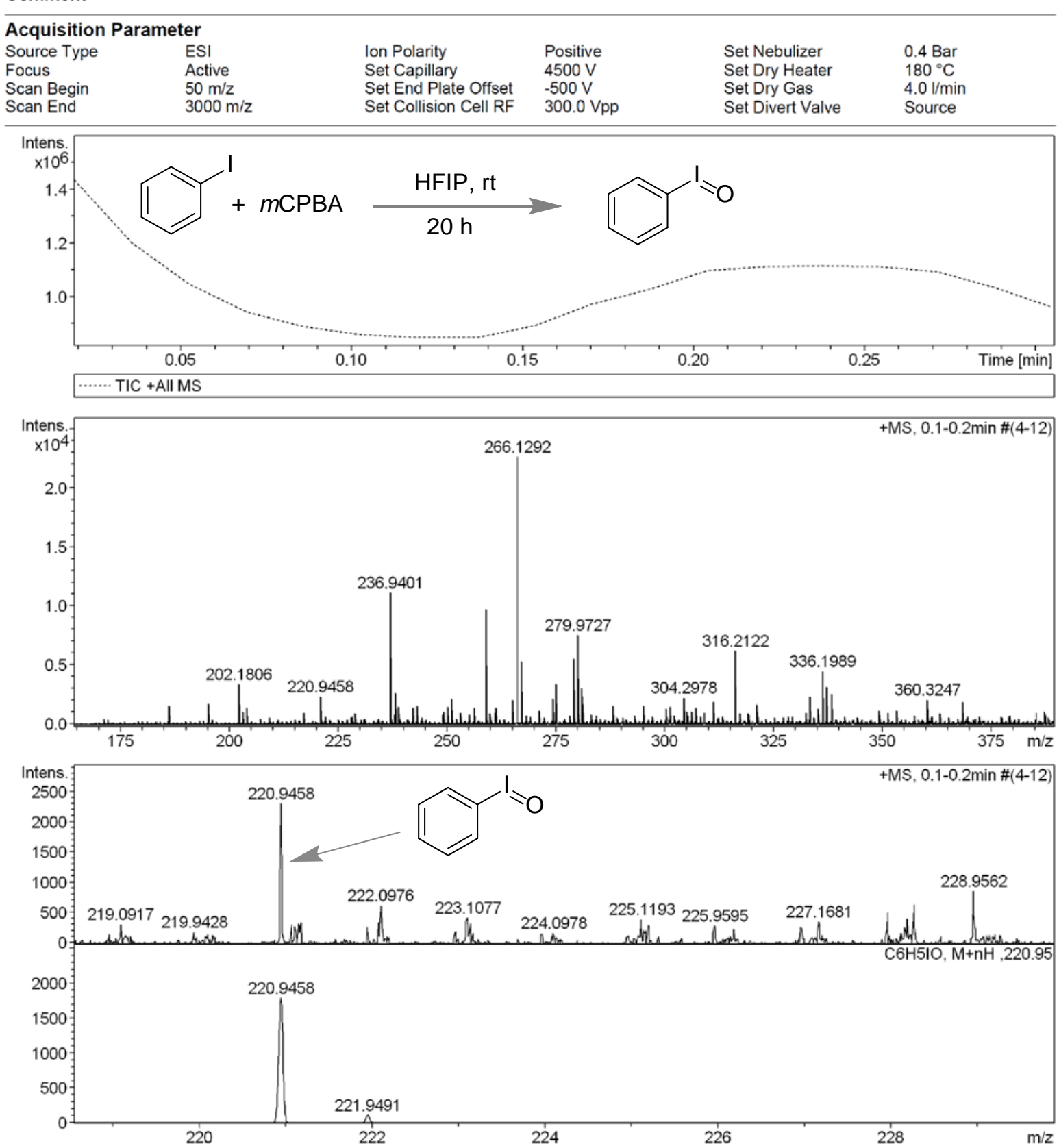

Bruker Compass DataAnalysis 4.0

printed: $\quad 8 / 22 / 201910: 05: 20 \mathrm{AM}$

Page 1 of 1

Figure S107. ESI-MS (HRMS) analysis of crude reaction mixture of $\mathrm{PhI}$ and $m \mathrm{CPBA}$. A peak at 220.9458 corresponds to PhIO. 